\title{
DENSE PARTICLE CLOUD DISPERSION BY A SHOCK WAVE
}

by

\author{
Mark Douglas Kellenberger
}

\author{
A thesis submitted to the \\ Department of Mechanical and Materials Engineering \\ In conformity with the requirements for \\ the degree of Master of Applied Science
}

\author{
Queen's University \\ Kingston, Ontario, Canada
}

September, 2012

Copyright (C) Mark Douglas Kellenberger, 2012 


\begin{abstract}
High-speed particle dispersion research is motivated by the energy release enhancement of explosives containing solid particles. In the initial explosive dispersal, a dense gas-solid flow can exist where the physics of phase interactions are not well understood. A dense particle flow is generated by the interaction of a shock wave with an initially stationary packed granular bed. The initial packed granular bed is produced by compressing loose aluminum oxide powder into a 6.35 $\mathrm{mm}$ thick wafer with a particle volume fraction of 0.48 . The wafer is positioned inside the shock tube, uniformly filling the entire cross-section. This results in a clean experiment where no flow obstructing support structures are present. Through high-speed shadowgraph imaging and pressure measurements along the length of the channel, detailed information about the particleshock interaction was obtained. Due to the limited strength of the Mach 2 incident shock wave, no transmitted shock wave is produced. The initial "solid-like" response of the particle wafer acceleration forms a series of compression waves that coalesce to form a shock wave. Breakup is initiated along the periphery of the wafer as the result of shear that forms due to the fixed boundary condition. Particle break-up starts at local failure sites that result in the formation of particle jets that extend ahead of the accelerating, largely intact, wafer core. In a circular tube the failure sites are uniformly distributed along the wafer circumference. In a square channel, the failure sites, and the subsequent particle jets, initially form at the corners due to the enhanced shear. The wafer breakup subsequently spreads to the edges forming a highly non-uniform particle cloud.
\end{abstract}




\section{Acknowledgements}

First and foremost, I would like to thank my supervisor, Dr. Gaby Ciccarelli, for his support. His continuous insight and guidance over the last two and a half years was essential to this project. For taking me on as a fourth year undergraduate student and introducing me to experimental gas dynamics and combustion, I cannot thank him enough.

I also must thank Dr. Craig Johansen for the patience he showed, and the time he took to teach me everything he could. His success is something that I look up to and I am grateful for all his advice.

Peter Diakow, Christopher Spaeth, and Thomas Pinos were valuable colleagues and friends in the combustion lab. Their feedback and ideas were always appreciated.

The construction of my apparatus would have been impossible without the help of the machine shop staff. Andy Bryson, Corey Fowler, Derek Hodgson and Paul Moreland always stepped up to meet the demanding deadlines I set and never hesitated to teach me how to use a machine.

The Queen's Varsity Swim Team and the Kingston Blue Marlins Swim Club gave me a home in the pool and a place to escape to. I want to thank Coach Ken Anderson for giving me the opportunity to continue to swim this year. It was one of the most enjoyable seasons I have ever had and I can't remember a time when I was happier to be in the pool.

Finally, I need to thank my mom, dad, brother, and sister for their love and encouragement.

This work was supported by Defence Research and Development Canada. 


\section{Contents}

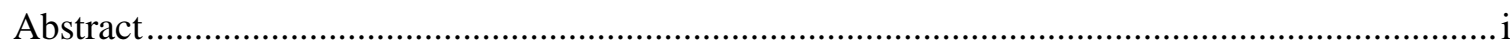

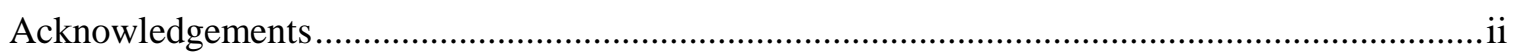

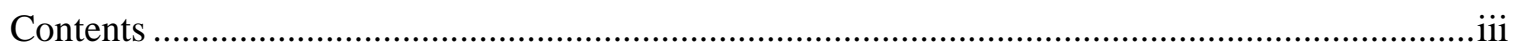

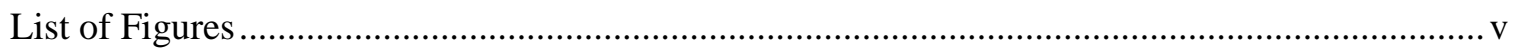

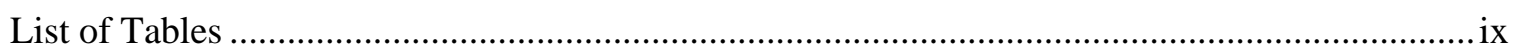

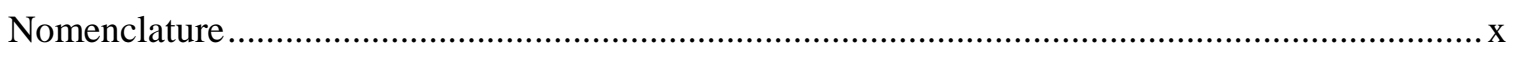

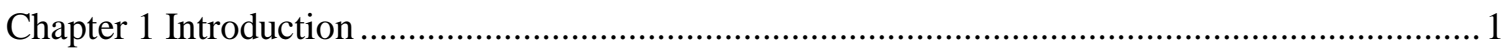

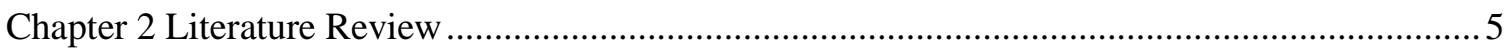

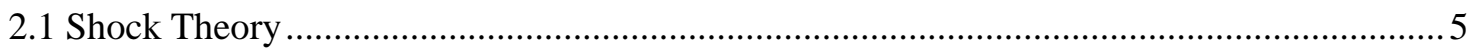

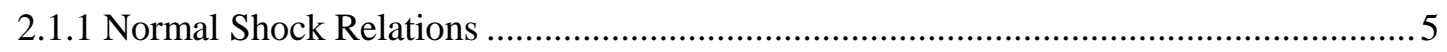

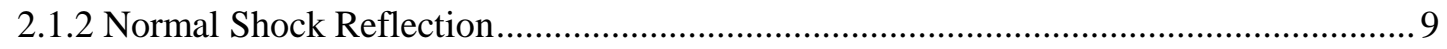

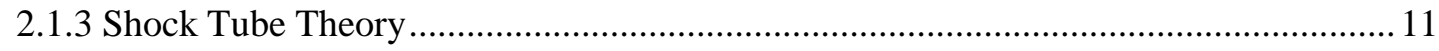

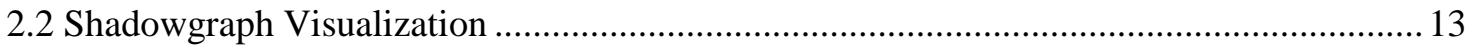

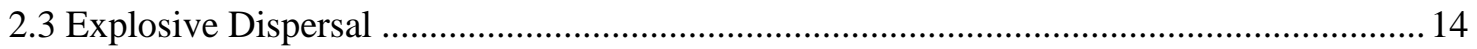

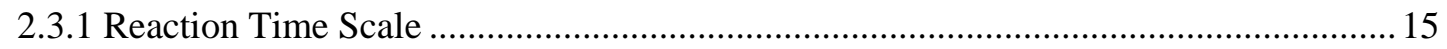

2.3.2 Factors Affecting Particle Penetration of Shock .......................................................... 17

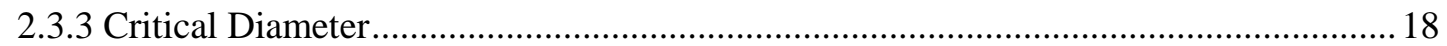

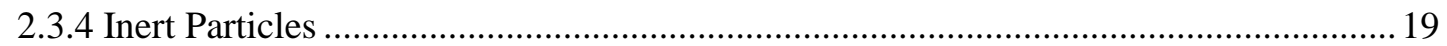

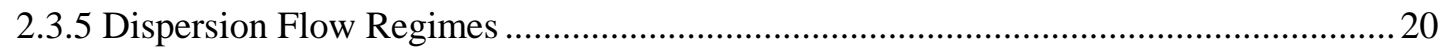

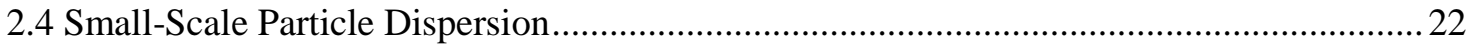

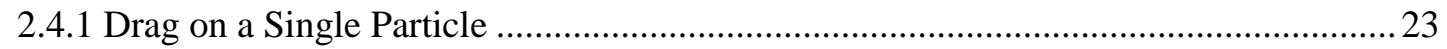

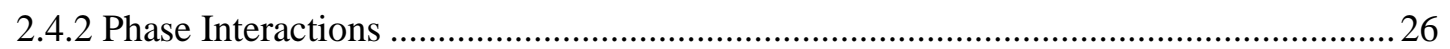

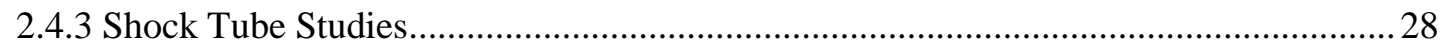

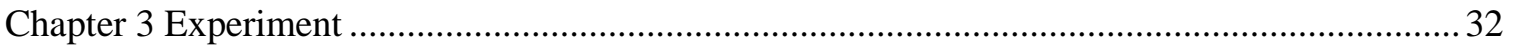

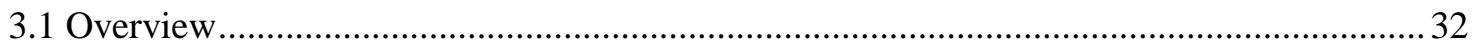

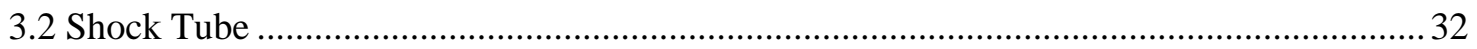

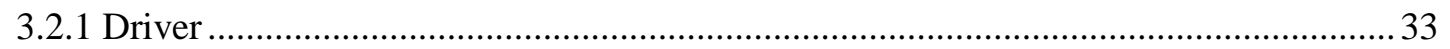

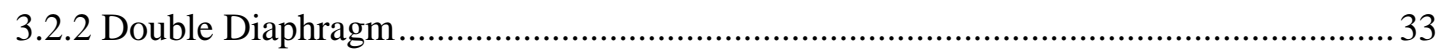

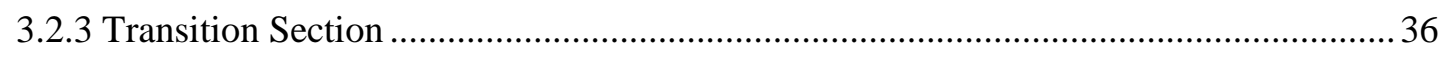

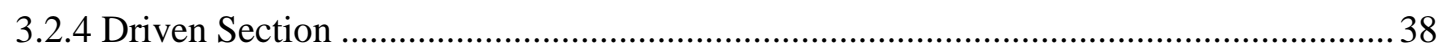

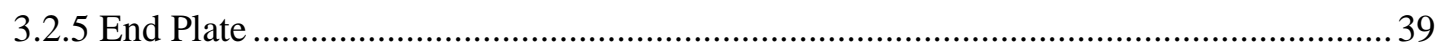




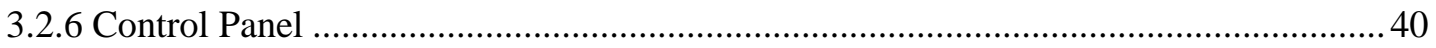

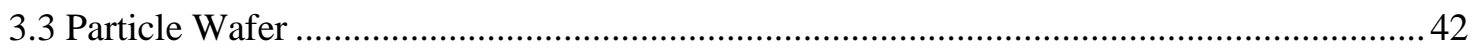

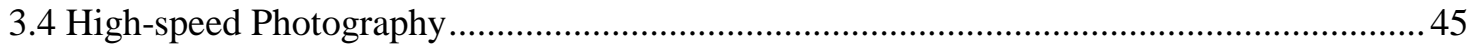

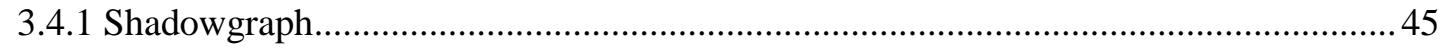

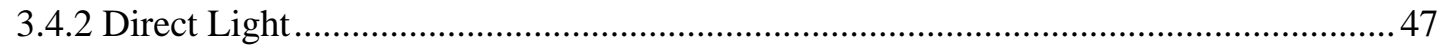

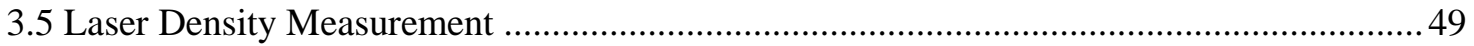

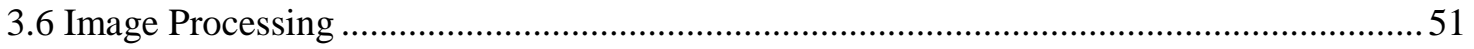

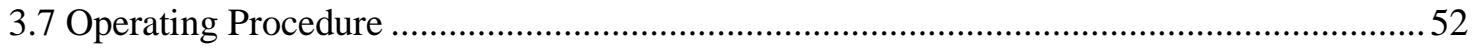

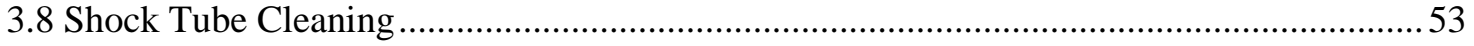

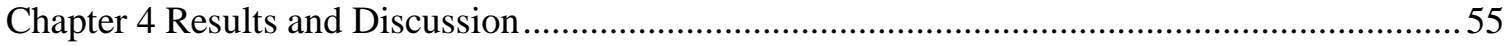

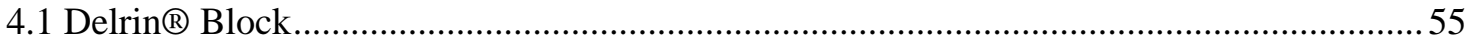

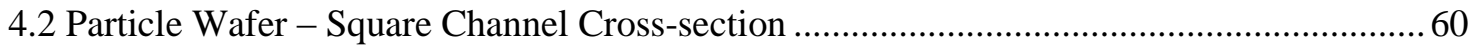

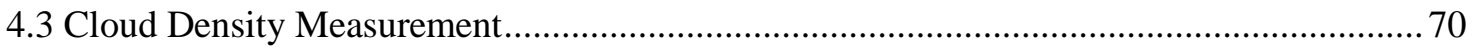

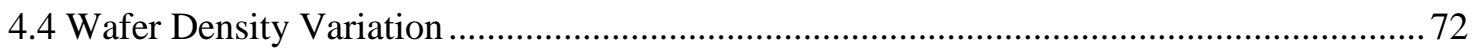

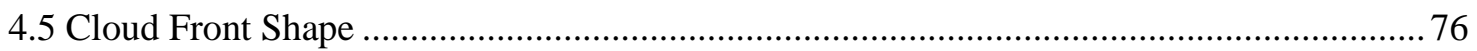

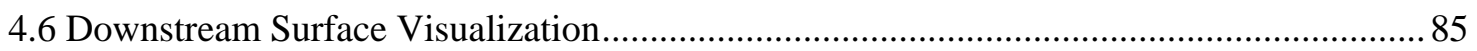

4.7 Particle Wafer - Circular Cross-section.......................................................................... 90

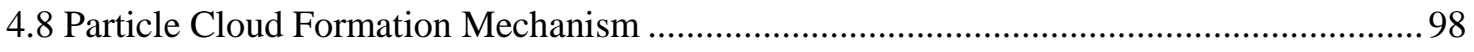

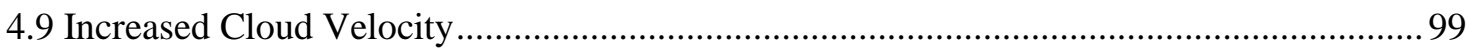

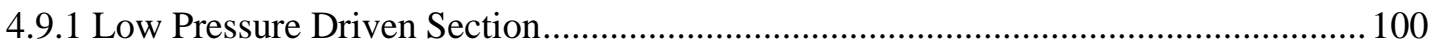

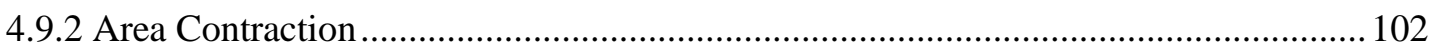

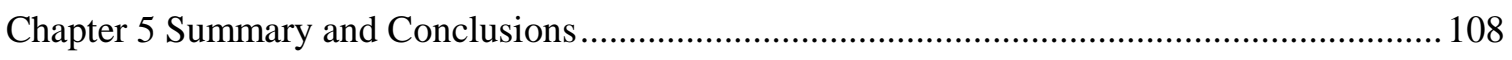

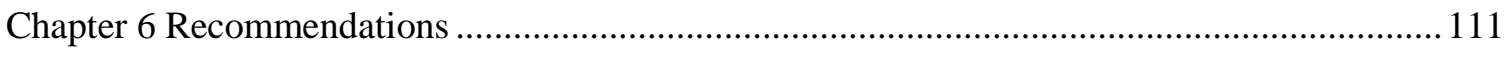

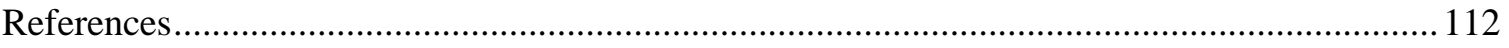

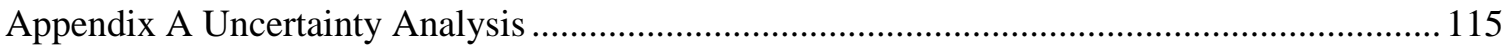

Appendix B MATLAB Cloud Tracking Code ....................................................................... 117 


\section{List of Figures}

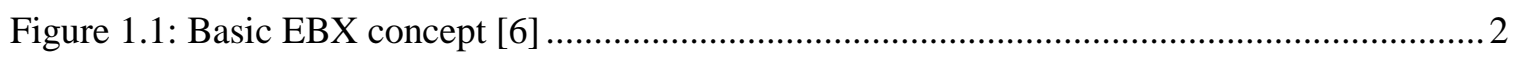

Figure 2.1: Normal shock wave control volume used in analysis................................................ 6

Figure 2.2: Rayleigh plot showing possible shock conditions [14] ........................................... 9

Figure 2.3: Normal shock reflection off the wall of a closed tube showing a) before reflection and

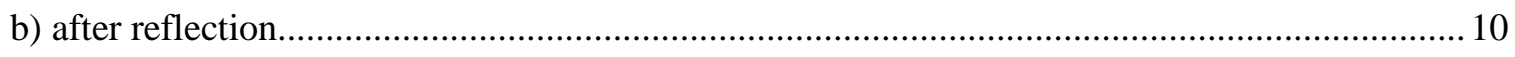

Figure 2.4: Schematic of shock tube after diaphragm rupture ................................................... 11

Figure 2.5: $x$-t diagram showing propagation of waves in shock tube after diaphragm rupture [15]

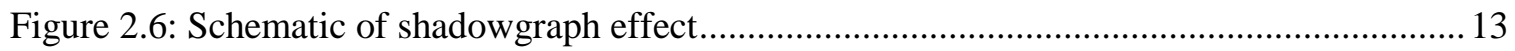

Figure 2.7: Schematic x-t diagram of detonation products flow field [4] .................................... 16

Figure 2.8: Comparison of pressure histories of conventional and EBX explosives ..................... 17

Figure 2.9: Critical charge diameter for particle ignition [16] ................................................. 19

Figure 2.10: Explosive dispersal of packed sand particles. Inter-frame time is $2.5 \mathrm{~ms}$ between the

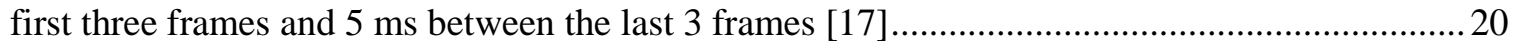

Figure 2.11: Dispersion flow regimes. The drag force acting on the particles, $F_{d}$, is normalized by the drag acting on a single sphere, $F_{d, S} . R_{0}$ is the charge radius [19] .......................................... 21

Figure 2.12: X-ray radiographs of a particle dispersal event. Images at $0 \mu \mathrm{s}, 43 \mu \mathrm{s}$ and $102 \mu \mathrm{s}$ [7]

Figure 2.13: Forces acing on an immersed body [20].

Figure 2.14: Deviation of drag coefficients in dense gas-solid flow regime from single particle drag coefficients. $C_{d}$ is the drag coefficient for a particle in a dense flow. $C_{d, s}$ is the drag coefficient for a single particle [19].

Figure 2.15: Shadowgraph images of dense gas-particle flow by a Mach 1.3 shock wave in air for a) a single layer of $2 \mathrm{~mm}$ glass particles at $2.65 \mathrm{~ms}$, b) a double layer of $2 \mathrm{~mm}$ diameter glass particles at $3.84 \mathrm{~ms}$, and c) a $2 \mathrm{~cm}$ thick bed of $1.5 \mathrm{~mm}$ diamter glass particles at $4.88 \mathrm{~ms}[9,19]$

28

Figure 2.16: Schlieren images of Mach 1.66 shock-particle curtain interaction [12] .................... 30

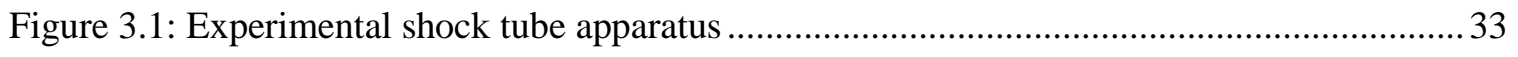

Figure 3.2: Double diaphragm holder in place at end of driver with Mylar diaphragms............... 34

Figure 3.3: Diaphragm setup and fill pressures for Mach 2.0 incident shock wave ..................... 35

Figure 3.4: Calibration test showing planar incident shock wave propagating from right to left.. 36 
Figure 3.5: Round to square shock tube transition section a) prior to welding and b) CAD drawing showing gradual cross-sectional change.

Figure 3.6: Schematic of shock tube showing the positions of the wafer, optical section, and the pressure transducers .38

Figure 3.7: Shock tube end plate enabling optical access from head-on perspective ................... 40

Figure 3.8: Schematic of shock tube control panel ................................................................... 41

Figure 3.9: a) Die used to compress powder into the plate cavity b) Particle wafer loaded in the

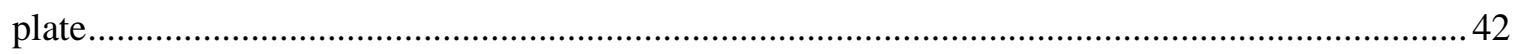

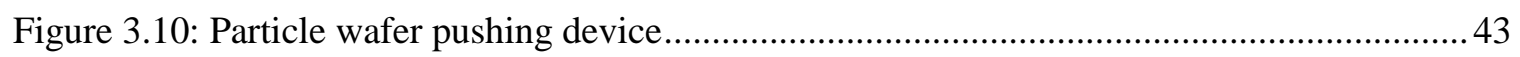

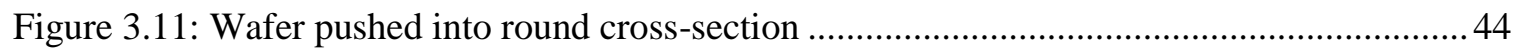

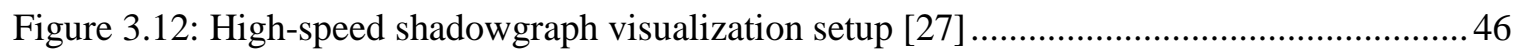

Figure 3.13: Camera and lighting setup for $30^{\circ}$ angle video .................................................... 48

Figure 3.14: Laser-based cloud density measurement setup showing a) photo of laser through

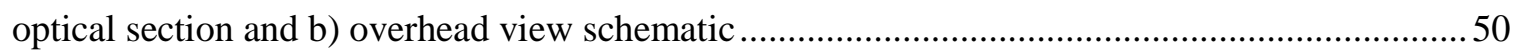

Figure 3.15: Air distributer used for cleaning powder from the inside of the shock tube .............54 Figure 4.1: Diagram showing pressure traces as well as shock and block trajectories obtained from video images for an 11 bar abs. helium driver with the camera field-of-view (see Figure 3.6) covering (a) the first half of the optical section and (b) the second half of the optical section. ....56 Figure 4.2: Images from 11 bar abs. helium driver showing acceleration of Delrin® block. Dark streak on gas due to abrasion from previous tests. Flow direction is from right to left .................58 Figure 4.3: Schematic showing shock wave-block impact event .60 Figure 4.4: Diagram showing pressure traces for a 6.5 bar abs. helium driver. Shock and cloud front trajectories from videos with the camera field-of-view (see Figure 3.6) covering (a) the first half of the optical section and (b) the second half of the optical section

Figure 4.5: Compilation of two videos showing the non-uniform particle cloud leading-edge and the shock wave development ahead of the particle cloud. From tests with 6.5 bar abs. helium driver. Inter-frame time is $0.027 \mathrm{~ms}$. Flow direction is from right to left.

Figure 4.6: Diagram showing pressure traces for an 11 bar abs. helium driver. Shock and block trajectories with the camera field-of-view covering a) the first half of the optical section and b) the second half of the optical section

Figure 4.7: Reflected shock comparison between a) particle wafer and b) Delrin® block from a 11 bar abs. helium driver. Inter-frame time is $0.134 \mathrm{~ms}$ 69 Figure 4.8: Diagram showing pressure traces for a 6.5 bar abs. helium driver with a $5.47 \mathrm{~cm}$ thick high-density foam block .71 
Figure 4.9: Cloud front velocity for varying particle volume fractions of initial wafer .73

Figure 4.10: Pressure trace taken comparing pressure wave structure of a) $\varphi_{p}=0.38$ and b) $\varphi_{p}=$ 0.58 particle wafers

Figure 4.11: Modified particle wafer with $12.7 \mathrm{~mm}$ sidewall gaps a) vertically oriented and b)

horizontally oriented .77

Figure 4.12: Images showing the shock wave and particle cloud dispersion. Results for 11 bar abs. driver pressure with $12.7 \mathrm{~mm}$ gap on sidewall wafer edges as shown in Figure 4.11a..... .79

Figure 4.13: Images showing the complex shock wave structure and particle cloud dispersion.

Results for 11 bar abs. driver pressure with $12.7 \mathrm{~mm}$ gap on top and bottom wafer edges as shown in Figure 4.11b. .80

Figure 4.14: Composite image constructed from Figure 4.12 and Figure 4.13 .81

Figure 4.15: Images showing shock and particle dispersion from the top of a Delrin® block following the interaction of shock wave produced by 11 bar abs. driver (12.7 mm gap on top and bottom)

Figure 4.16: Results for 11 bar abs. driver pressure with $12.7 \mathrm{~mm}$ gap on sidewall wafer edges 84 Figure 4.17: Images showing the initial wafer breakup and cloud development for $11 \mathrm{bar}$ abs. driver pressure.

Figure 4.18: Head-on view showing initial wafer breakup with degradation zone and corner failure as well as cloud development for 11 bar abs. driver pressure 87

Figure 4.19: Powder within die before and after compaction showing forces on plate sidewalls once compressed.

Figure 4.20: Particle wafer showing areas where movement is restricted due to plate sidewall effects in red and corner failure zones in blue 90

Figure 4.21: Side-view of 11 bar abs. driver pressure round cross-section 91

Figure 4.22: Head-on view showing initial wafer breakup and cloud development in a circular tube for 11 bar abs. driver pressure.

Figure 4.23: Head-on view for 11 bar abs. driver pressure showing initial wafer breakup in round cross-section with a pushed-in particle wafer .94

Figure 4.24: Particle cloud leading-edge for pushed-in wafer in round cross-section. From tests with 11 bar abs. helium driver .96

Figure 4.25: Experimental cloud front velocities from square and round cross-section. 97 Figure 4.26: Results for 11 bar abs. helium driver pressure with 0.5 bar abs. atmospheric air driven section pressure. 
Figure 4.27: Images from 11 bar abs. helium driver with opposing $18.4^{\circ}$ ramps before particle wafer.

Figure 4.28: Schematic of tube setup for area contraction tests .....

Figure 4.29: Images from 11 bar abs. driver with opposing $18.4^{\circ}$ ramps before particle wafer, channel height of $2.54 \mathrm{~cm}$. Camera frame rate of $105000 \mathrm{fps}$ 106 


\section{List of Tables}

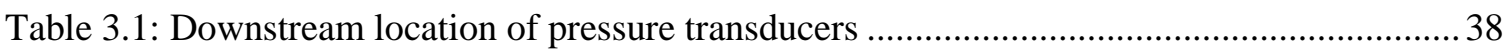




\section{Nomenclature}

$\begin{array}{ll}\text { Abbreviations } & \\ \text { CAD } & \text { computer-aided design } \\ \text { CDPI } & \text { critical diameter for particle ignition } \\ \text { CMOS } & \text { complimentary metal-oxide semi-conductor } \\ \text { DAQ } & \text { data acquisition } \\ \text { EBX } & \text { enhanced blast explosive } \\ \text { RAM } & \text { random access memory } \\ \text { TBX } & \text { thermobaric explosive } \\ \text { TTL } & \text { transistor-transistor logic }\end{array}$

\section{English Symbols}

$\begin{array}{ll}a & \text { speed of sound }[\mathrm{m} / \mathrm{s}] \\ c & \text { speed of light }[\mathrm{m} / \mathrm{s}] \\ C_{D} & \text { drag coefficient } \\ c_{p} & \text { specific heat capacity at constant pressure }[\mathrm{J} / \mathrm{kg} \cdot \mathrm{K}] \\ D & \text { Diameter }[\mathrm{m}] \\ f & \text { frame rate }[1 / \mathrm{s}] \\ F_{D} & \text { drag force }[\mathrm{N}] \\ F_{L} & \text { lift force }[\mathrm{N}] \\ F_{n} & \text { normal force }[\mathrm{N}] \\ F_{t} & \text { tangential force }[\mathrm{N}] \\ h & \text { Enthalpy }[\mathrm{kJ} / \mathrm{kg}] \\ k & \text { specific heat ratio }\end{array}$




$\begin{array}{ll}M & \text { Mach number } \\ n & \text { refractive index } \\ P & \text { Pressure [Pa] } \\ \bar{R} & \text { ideal gas constant }[\mathrm{J} / \mathrm{mol} \cdot \mathrm{K}] \\ R & \text { Radius [m] } \\ R e & \text { Reynolds number } \\ T & \text { Temperature [K] } \\ t & \text { Time [s] } \\ u & \text { flow velocity [m/s] } \\ V & \text { wave velocity [m/s] } \\ x & \text { downstream distance [m] }\end{array}$

\section{Greek Symbols}

$\begin{array}{ll}\alpha & \text { pixel density [pixles } / \mathrm{m}] \\ \beta & \text { refractivity constant } \\ \varepsilon & \text { voltage drop [V] } \\ \kappa & \text { pixels from the start of the field of view to cloud front } \\ \mu & \text { Gladstone-Dale constant } \\ \pi & \text { dynamic viscosity }[\mathrm{kg} / \mathrm{m} \cdot \mathrm{s}] \\ \rho & \text { Pi } \\ \sigma_{A} & \text { Density }\left[\mathrm{kg} / \mathrm{m}^{3}\right] \\ \sigma_{L} & \text { axial pressure }[\mathrm{Pa}] \\ \varphi & \text { lateral pressure }[\mathrm{Pa}]\end{array}$




\section{Chapter 1}

\section{Introduction}

The study of particle cloud dispersion by a shock wave is important to many applications. For example, the propagation of shock wave through a dusty gas has been studied for many years [1]. In these studies the particle volume fraction is insignificant and the particle acceleration can be modeled using simple drag correlations based on a sphere in an infinite medium. A modern application includes multiphase explosives that comprise of a condensed explosive surrounded by packed micrometric reactive metal particles.

It is now common practice to add reactive metals (aluminum or magnesium) to condensed explosives. The metals are often included as a flash powder, which is becoming an increasingly common additive to explosives, in order to increase the total energy release of the explosion [2]. The large surface area of the fine powder makes it highly reactive when exposed to oxygen and these metallic particles can add large amounts of energy to explosions [3]. The shock wave from a high explosive charge has the potential to ignite these tiny particles. The ignition of the particles significantly increases the strength of the blast wave by up to six times [4]. Explosives of this type are commonly referred to as Enhanced Blast Explosives (EBX). Another common name for the same type of device is a Thermobaric Explosive (TBX).

The basic setup of an EBX consists of a condensed explosive surrounded with packed micrometric reactive metal particles [5]. The shock wave from the detonation wave in the explosive breaks the thin shell containing the charge and disperses the particles in the combustion

products (fireball). Some energy is released from the particles as they react with the water in the combustion products, but most of the energy is released later in time when the hot metal particles mix with the air outside the fireball. Figure 1.1 shows a schematic of the basic EBX concept. 


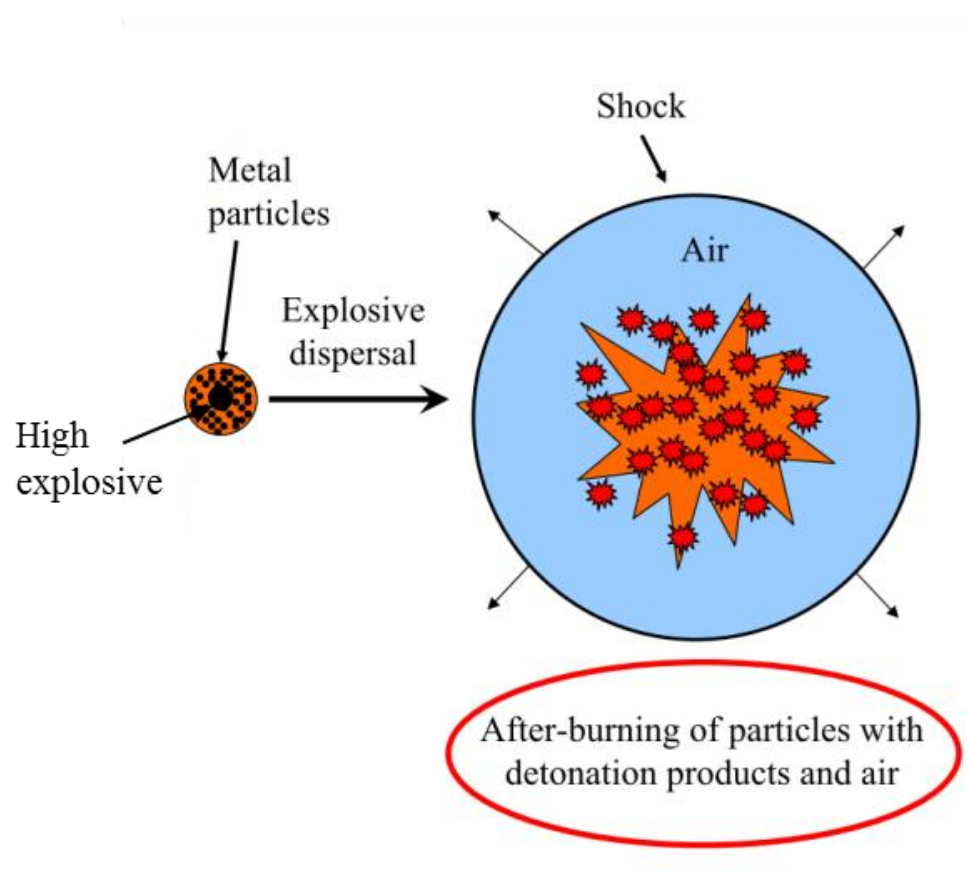

\section{Figure 1.1: Basic EBX concept [6]}

Shock and particle reflection off solid structures can cause enhanced energy release due to higher air temperatures and fragmentation of the metal particles.

The particle dispersion in an explosive dispersal application is very complicated as the gas-particle interaction transitions through multiple regimes, each requiring a different treatment. The solid volume fraction, $\varphi_{p}$, of the flow is used to identify the regimes [7]. In a granular bed $\left(\varphi_{p}\right.$ $>0.5)$, forces are exerted also through direct contact of the closely packed particles. In a dilute gas-solid flow $\left(\varphi_{p}<0.01\right)$, the effects of particle collisions become negligible. Between these two regimes is a dense gas-solid flow $\left(0.1<\varphi_{p}<0.5\right)$. Detonation of the explosive generates a shock wave that accelerates and compacts the particle bed and when the shock reaches the particle layer back boundary, a reflected expansion wave further accelerates the particles. The shock wave initially propagates through a granular particle bed and much later, after the passage of the shock wave, the particles are further dispersed by the radial combustion product flow forming a cloud. In this application it is important to understand the dispersion of the particles as the late-time 
energy release from the particles maintains the pressure in the shock sphere, thereby increasing the shock wave impulse [8].

The particle dispersion phenomenon varies from early time when the particle volume fraction is large, to later time when the volume fraction is very small and the flow is considered a dilute gas-solid flow, i.e., similar to a dusty-gas flow. The particle dispersion phenomenon responsible for very late time dispersion has been studied extensively and is relatively well understood. However, for the early time dispersion of a granular bed, as the particle cloud expands, a dense gas-solid flow exists which has not received much attention and is not very well understood [7].

Reactive multiphase flow models have been used to simulate the acceleration and dispersion of the particle cloud that occurs in multiphase explosives [9]. The fidelity of the simulations is severely limited by the physical drag models that take into account the interactions in the dense gas-solid flow [7]. In order to develop an accurate particle drag model that describes particle acceleration in the dense flow regime, representative experimental data need to be generated. Large-scale experiments have been carried out with spherical multiphase explosive charges to visualize the global particle cloud dispersion [10]. Controlled shock wave experiments are necessary to gain understanding of the fundamental physics of the dense supersonic gas-solid flow interactions and obtain quantitative data concerning the particle cloud dispersion. Shock tube experiments have been carried out in the past but typically the particle suspension method influences the shock flow and the particle size is not typical of multiphase explosives. Tests performed by Rogue et al. [9] used a plastic membrane to support $1 \mathrm{~mm}$ to $2 \mathrm{~mm}$ particles in a vertical shock tube. Similarly, Anilkumar [11] used a vertical tube and a lofting method to introduce particles. Recently, shock tube experiments were carried out by Wagner et al. [12] looking at the interaction of a shock wave and a gravity-fed particle "curtain". 
This thesis reports on shock tube experiments investigating the acceleration and dispersion of 100 micron-sized aluminum oxide particles [5]. By pure coincidence the experimental conditions are very similar to those of the Wagner et al. [12] experiment, i.e., similar shock tube cross-section dimensions, shock wave strength, and particle size. The main difference between the experiments is in the initial particle cloud volume density. The novel feature of the present experiments is the initial configuration of the particles in the form of a freestanding wafer covering the entire shock tube cross-section. This method allows absolute control over the initial particle distribution without the use of flow obstructing structures. 


\section{Chapter 2}

\section{Literature Review}

\subsection{Shock Theory}

\subsubsection{Normal Shock Relations}

In a gas flow, properties of the gas can change when the flow velocity is high because of compressibility effects. In general, gases are highly compressible however, compressibility effects can be negligible if the Mach number is low enough as density changes in the flow are then small. Where the Mach number becomes greater than about 0.3 , the flow can no longer be considered incompressible. The Mach number, $M$, is defined as the ratio of the velocity to the speed of sound.

$$
M=\frac{u}{a}
$$

Where $a$ is the speed of sound in a perfect gas and is given by

$$
a=\sqrt{k \bar{R} T}
$$

Where $k$ is the specific heat ratio and the gas is assumed to be perfect, with $\bar{R}$ being the gas constant. If the Mach number for a given flow is greater than 1, the flow is said to be supersonic, while a Mach number of $M<1$, the flow is said to be subsonic. In a supersonic flow, effective discontinuities can arise in the flow field because of compressibility effects. These are known as shock waves and are characterized by a sharp pressure, density and temperature rise, and a sharp drop in velocity. The shock wave is very thin, typically a few mean free paths thick [13]. This is a few times the average distance a molecule has to travel between collisions in the gas. 
The simplest type of shock wave is one that forms at a right angle to the flow direction and are known as normal shock waves. Using a control volume approach, property changes across the shock wave in a perfect gas can be determined.

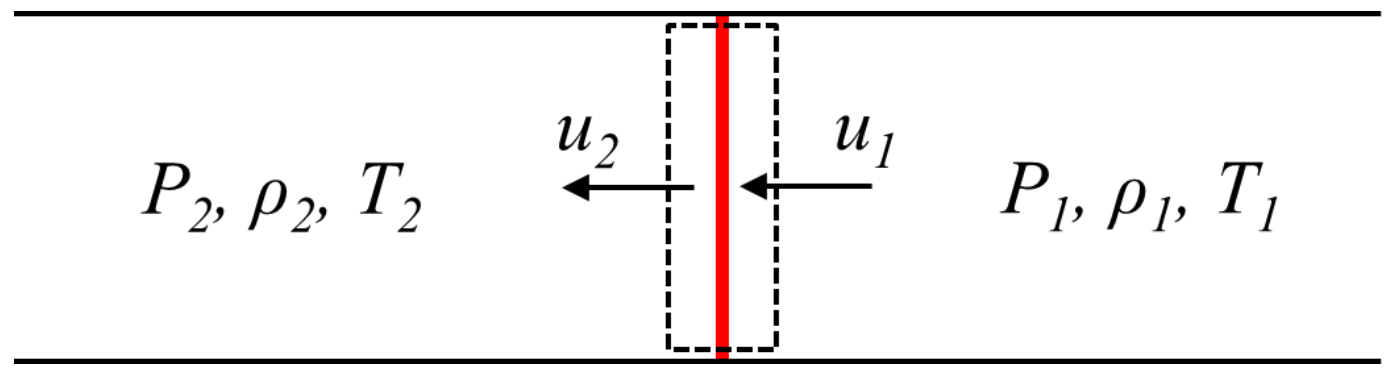

Figure 2.1: Normal shock wave control volume used in analysis

Conservation of mass, momentum, and energy must apply through the control volume shown in Figure 2.1.

$$
\begin{gathered}
\rho_{1} u_{2}=\rho_{2} u_{2} \\
P_{1}-P_{2}=\rho_{1} u_{1}\left(u_{1}-u_{2}\right) \\
\frac{u_{1}^{2}}{2}+h_{1}=\frac{u_{2}^{2}}{2}+h_{2}
\end{gathered}
$$

In a perfect gas,

$$
\begin{gathered}
h=c_{p} T=\frac{k \bar{R}}{k-1} T=\frac{k P}{\rho(k-1)} \\
P=\rho \bar{R} T
\end{gathered}
$$


From Equation 2.5 and the perfect gas relations, assuming no change in the specific heat ratio, $k$, the pressure ratio (shock strength) can be found by,

$$
\frac{P_{2}}{P_{1}}=\frac{\left[\left(\frac{k+1}{k-1}\right) \frac{\rho_{2}}{\rho_{1}}-1\right]}{\left[\left(\frac{k+1}{k-1}\right)-\frac{\rho_{2}}{\rho_{1}}\right]}
$$

Rearranging Equation 2.8 gives the density ratio across the shock wave

$$
\frac{\rho_{2}}{\rho_{1}}=\frac{\left[\left(\frac{k+1}{k-1}\right) \frac{P_{2}}{P_{1}}+1\right]}{\left[\left(\frac{k+1}{k-1}\right)+\frac{P_{2}}{P_{1}}\right]}
$$

Rearranging Equation 2.3 and substituting into Equation 2.9 gives the velocity ratio across the shock wave,

$$
\frac{u_{1}}{u_{2}}=\frac{\left[\left(\frac{k+1}{k-1}\right) \frac{P_{2}}{P_{1}}+1\right]}{\left[\left(\frac{k+1}{k-1}\right)+\frac{P_{2}}{P_{1}}\right]}
$$

From Equation 2.7 and 2.9, the temperature ratio across the shock wave is

$$
\frac{T_{2}}{T_{1}}=\frac{\left[\left(\frac{k+1}{k-1}\right)+\frac{P_{1}}{P_{2}}\right]}{\left[\left(\frac{k+1}{k-1}\right)+\frac{P_{1}}{P_{2}}\right]}
$$

The preceding three equations are known as the Rankine-Hugoniot normal shock wave relations, giving the density, velocity and temperature ratios across a normal shock wave in terms of the shock strength, i.e. shock pressure ratio.

To relate the downstream Mach number to the upstream Mach number, Equation 2.2 can be combined with Equation 2.3, giving the conservation of mass in terms of Mach number. Substituting this into Equation 2.4 gives, 


$$
\frac{\rho_{2}}{\rho_{1}}=\left(\frac{1+k M_{1}^{2}}{1+k M_{2}^{2}}\right)\left(\frac{a_{2}}{a_{1}}\right)^{2}
$$

From Equation 2.12 and the conservation of energy in Equation 2.5 it can be found that,

$$
\left(\frac{a_{2}}{a_{1}}\right)^{2}=\left[\frac{2+(k-1) M_{1}^{2}}{2+(k-1) M_{2}^{2}}\right]
$$

Combining Equation 2.12 and Equation 2.13 gives the density ratio across the shock wave in terms of the Mach number.

$$
\frac{\rho_{2}}{\rho_{1}}=\frac{(k+1) M_{1}^{2}}{2+(k-1) M_{1}^{2}}
$$

It follows that,

$$
\begin{gathered}
\frac{P_{2}}{P_{1}}=\frac{2 k M_{1}^{2}-(k-1)}{(k+1)} \\
\frac{T_{2}}{T_{1}}=\left(\frac{a_{2}}{a_{1}}\right)^{2}=\frac{\left[2 k M_{1}^{2}-(k-1)\right]\left[2+(k-1) M_{1}^{2}\right]}{(k+1)^{2} M_{1}^{2}} \\
M_{2}=\sqrt{\frac{(k-1) M_{1}^{2}+2}{2 k M_{1}^{2}+(k-1)}}
\end{gathered}
$$

Results from the above equations are often tabulated for ease of use, for given $M_{l}$ and $k$ values.

Combining Equation 2.3 and Equation 2.4 gives,

$$
P_{2}-P_{1}=\left(\rho_{1} u_{1}\right)^{2}\left(\frac{1}{\rho_{1}}-\frac{1}{\rho_{2}}\right)
$$

Plotting Equation 2.18 on a normalized pressure versus normalized density plot, as shown in Figure 2.2, gives a straight line commonly referred to as the Rayleigh line. 


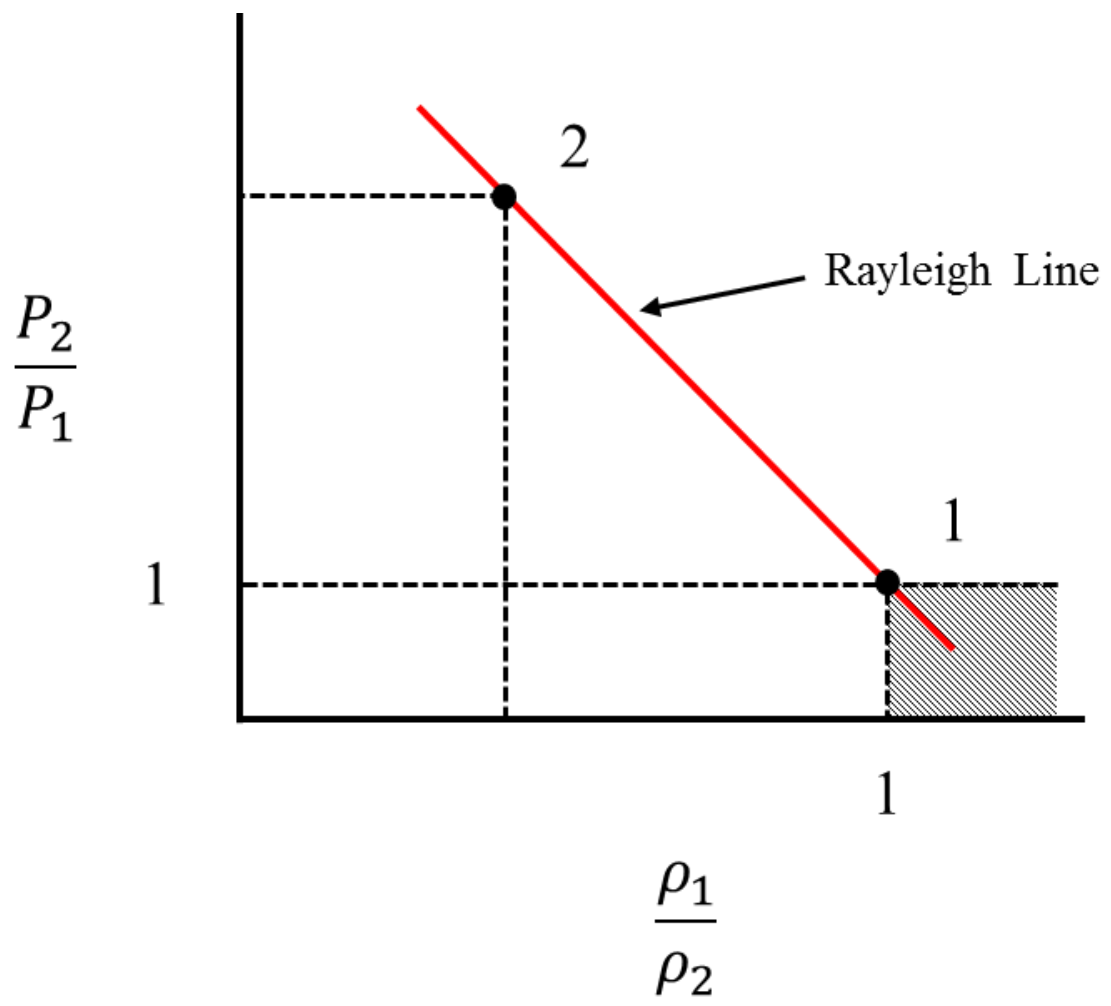

Figure 2.2: Rayleigh plot showing possible shock conditions [14]

A pressure ratio less than 1 is not possible if the density ratio is greater than 1 . This is because for that to happen, the entropy across the wave would have to decrease and that is not possible according to the second law of thermodynamics. Therefore, an "expansion shock" is not possible in nature.

\subsubsection{Normal Shock Reflection}

In a closed-end tube, a normal shock wave will reflect off the end of the tube. The incident shock propagates through the gas that is initially at rest (state 1 in Figure 2.3), inducing a flow velocity behind the shock wave in the same direction of the shock propagation (state 2 in Figure 2.3). Since there can be no flow out the end of the tube, the shock wave must reflect off the end, bringing the flow velocity back to zero (state 3 in Figure 2.3). 


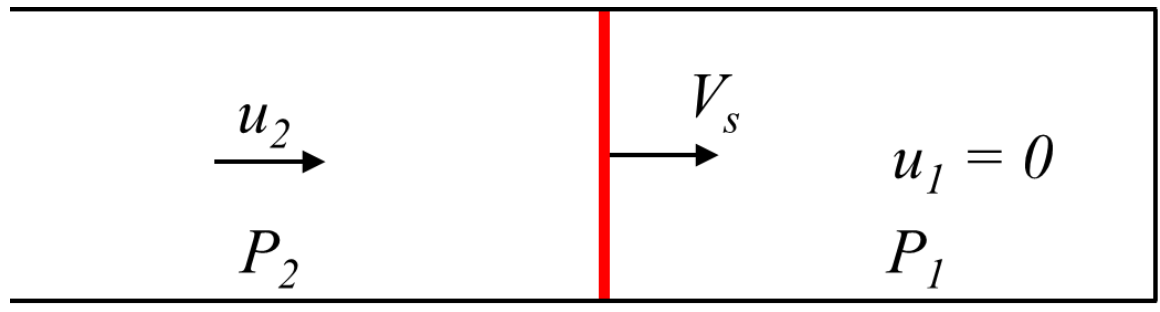

(a)

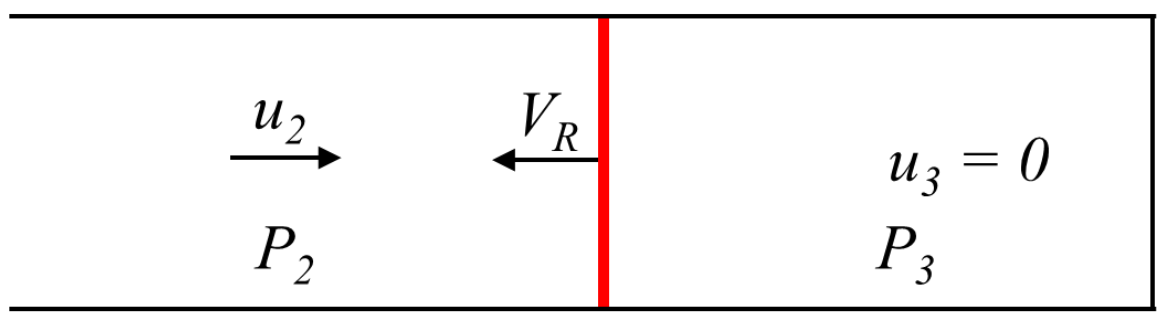

(b)

Figure 2.3: Normal shock reflection off the wall of a closed tube showing a) before reflection and b) after reflection

The gas velocity behind the reflected shock must be zero to satisfy conservation laws. Following Oosthuizen and Carscallen [13] to satisfy this, the Mach numbers upstream and downstream of the reflected shock wave are given by,

$$
\begin{gathered}
M_{u p}=\frac{\left(u_{2}+V_{R}\right)}{a_{2}}=M_{2}+\frac{V_{R}}{a_{2}} \\
M_{\text {down }}=\frac{V_{R}}{a_{3}}
\end{gathered}
$$

Using the normal shock equations presented in Section 2.1.1, the upstream and downstream Mach numbers can be related by,

$$
M_{\text {down }}=\left(M_{u p}-M_{2}\right) \sqrt{\frac{T_{2}}{T_{3}}}
$$


In order to calculate $V_{R}$ for a given $M_{1}$, an iterative process can be used. A value for $M_{u p}$ is guessed and $M_{\text {down }}$ is calculated using Equation 2.20 by substituting in Equation 2.17 for $M_{2}$ and Equation 2.16 for the temperature ratio. This allows for a value of $M_{\text {down }}$ to be found in terms of only $M_{l}$ and $M_{u p}$. The result is then compared to the value of $M_{\text {down }}$ calculated using the shock jump relation of Equation 2.17. When the two $M_{\text {down }}$ values are equal, the correct $M_{u p}$ value has been found. Once the reflected shock velocity is known, the reflected pressure, $P_{3}$, can be calculated using the normal shock relations.

\subsubsection{Shock Tube Theory}

A shock tube is used to create a shock wave in a controlled environment. A shock tube consists of two sections: a driver and a driven section. Separating these high (driver) and low (driven) pressure sections is a diaphragm. To generate strong shock waves, the driver gas is usually chosen with a high speed of sound. A large pressure differential across the diaphragm causes it to rupture. The expansion of the driver gas produces a shock wave that propagates supersonically into the driven section of the shock tube. This is shown in Figure 2.4.

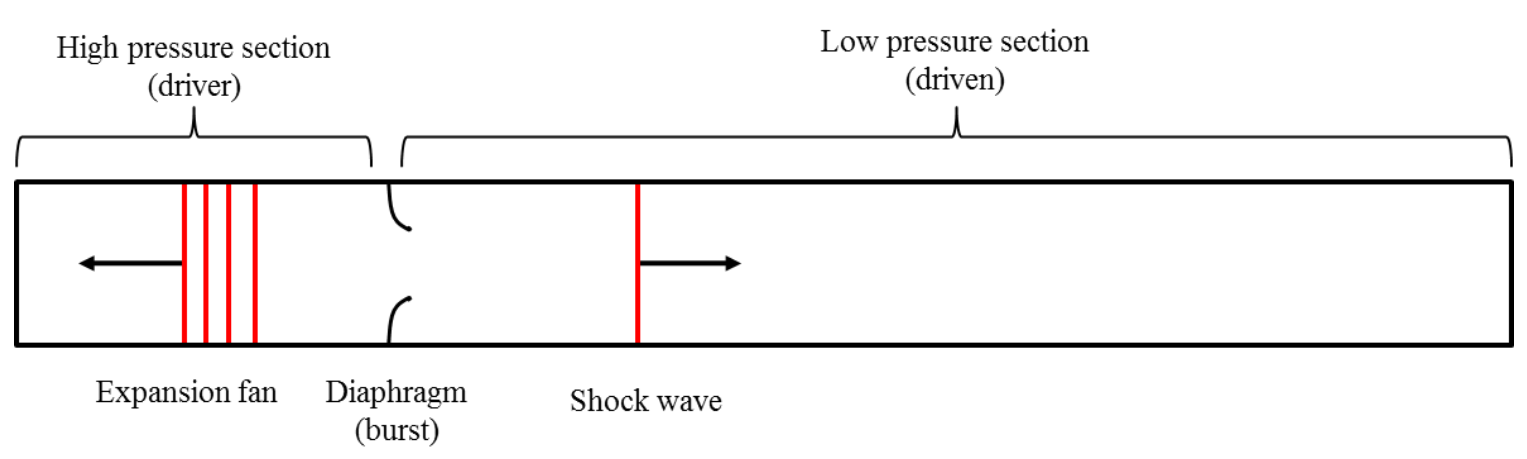

Figure 2.4: Schematic of shock tube after diaphragm rupture

Along with the shock wave, an expansion fan (consisting of a series of expansion waves) is formed that travels back upstream, into the driver. The head of the expansion fan travels at the 
speed of sound. The flow through the expansion fan is isentropic, unlike the shock wave that forms. Following behind the shock wave is the contact surface which is the interface between the driver gas and the driven gas, across which the pressure and velocity are constant and very little mixing occurs. An x-t diagram of the different waves that form after diaphragm rupture is shown in Figure 2.5.

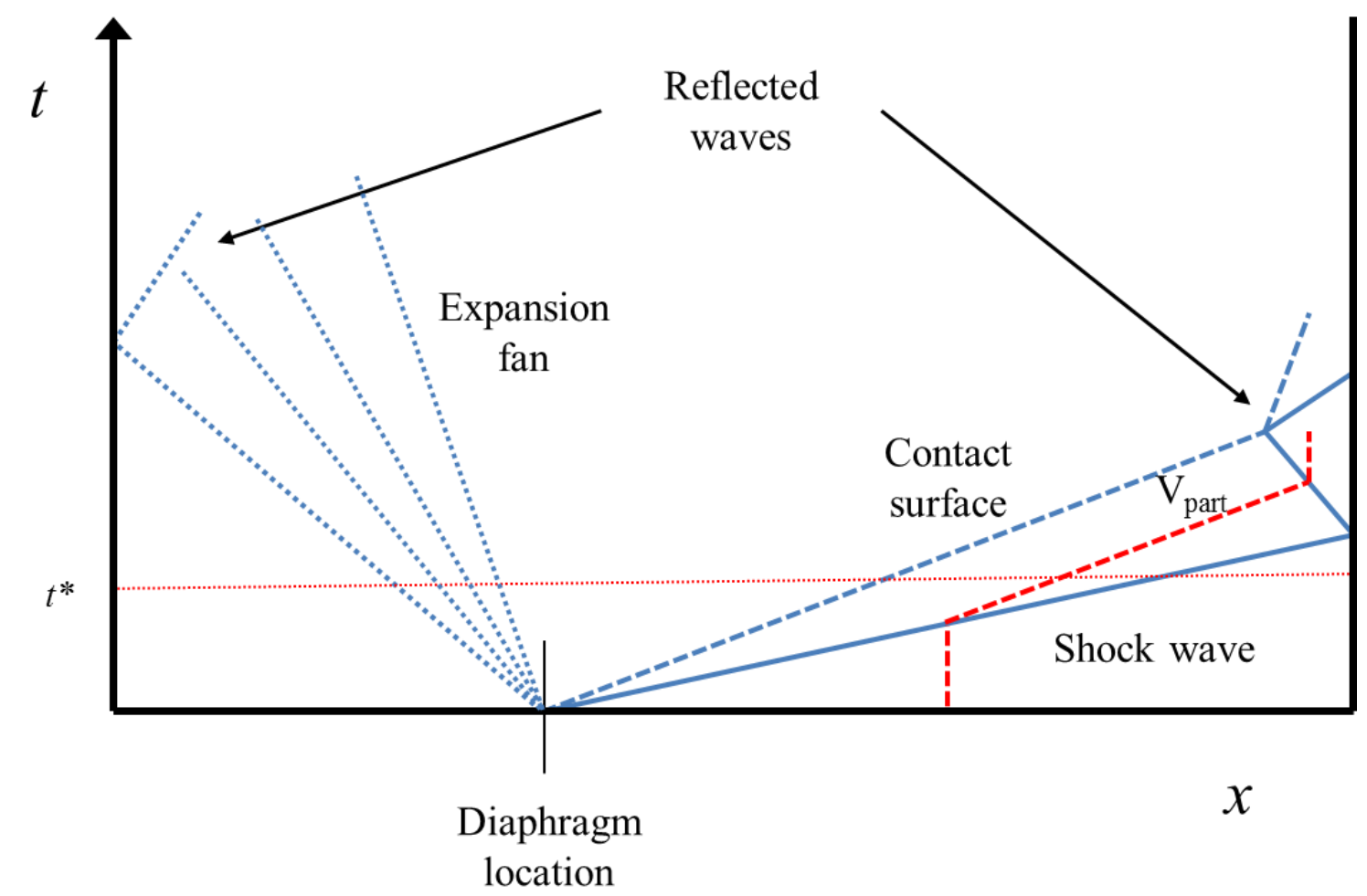

Figure 2.5: $x-t$ diagram showing propagation of waves in shock tube after diaphragm rupture [15]

The $\mathrm{x}-\mathrm{t}$ diagram is a common way of displaying wave trajectories where the slope of the line represents the wave velocity. Also shown in Figure 2.5 is the gas-particle trajectory that is vertical (stationary) before the passage of the incident shock wave and after the passage of the reflected shock wave. Between these two interactions it propagates at the same velocity as the 
contact surface. At any time $t^{*}$, as shown in Figure 2.5, the downstream distance of the shock wave can be determined along with its position relative to any other wave.

For the purposes of analysis, it is assumed that the diaphragm ruptures instantly. Although in practice there is a finite opening time over which a series of compression waves build up to form the shock wave. Diaphragms are made of a variety of materials, including plastics for low pressures and metals for higher pressures. Often the diaphragm is scored with an X-pattern so that it "petals", maximizing the opening area and minimizing transverse waves that can form.

\subsection{Shadowgraph Visualization}

Shadowgraph is a density-sensitive flow visualization technique that passes parallel light through the shock tube test section. Since the speed of light is dependent on the density of the medium that it is travelling through, density gradients cause the parallel light to be refracted and appear as light and dark spots on the camera sensor, depending on whether the light rays converge or diverge at a spot, as seen in Figure 2.6.

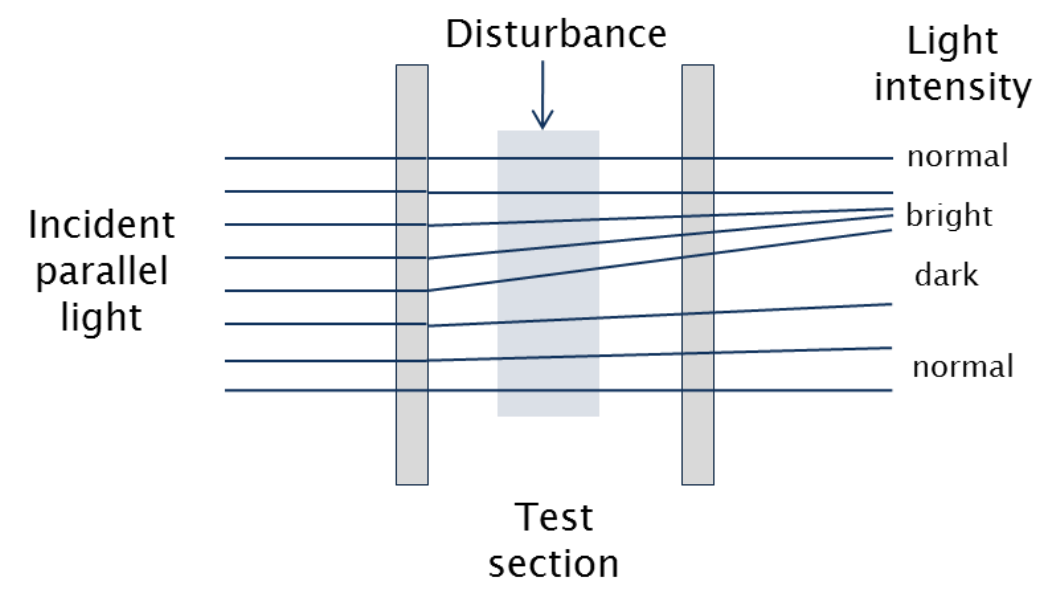

Figure 2.6: Schematic of shadowgraph effect 
Since shock waves by definition are a discontinuity in the medium density, shadowgraph photography detects the movement of these discontinuities. The speed of light, $c$, is at its maximum in a vacuum where the density is zero and while in a gas the speed of light is lower. The ratio of these two speeds of light is known as the refractive index,

$$
n=\frac{c_{0}}{c}
$$

Where $c_{0}$ is the speed of light in a vacuum and $c$ is the speed of light in a gas. Since the relationship between a medium's refractive index, $n$, and its density are approximately linear [13], the refractive index can be written in terms of density,

$$
n=1+\beta \frac{\rho}{\rho_{s}}
$$

Where $\beta$ is a constant, depending on the type of gas and $\rho_{s}$ is the density of the gas at atmospheric pressure and $0^{\circ} \mathrm{C}$. For air $\beta=2.92 \times 10^{-4}$ [13]. Often the refractive index is written in terms of the Gladstone-Dale coefficient, $\kappa$, where

$$
\kappa=\frac{\beta}{\rho_{s}}
$$

When a beam of light travels through a density gradient off-normal to the beam, the light is turned towards the normal of increased density (away from the normal for decreasing density) with the turning angle being dependent on the gradient of the density. It can be shown that the shadowgraph is sensitive to the second derivative of the refractive index [13],

$$
\frac{\partial^{2} n}{\partial x^{2}}
$$

\subsection{Explosive Dispersal}

In an explosive dispersion, detonation of the explosive generates a shock wave that accelerates and compacts the surrounding metal particle bed. The particles are further accelerated from the 
reflected expansion as the shock wave arrives at the free-surface of the particles. When the detonation front first arrives at a particle, momentum is imparted by pressure and viscous forces and the temperature is raised by the high-temperature combustion products [4]. It has also been observed that certain size particles may have enough momentum to catch and penetrate the decelerating contact surface (fireball), see Figure 2.7. Smaller particles will accelerate to the flow velocity of the combustion products very quickly, where the large inertia of bigger particles will cause them respond more slowly. Some particles of an intermediate size will experience the effects of a decaying blast wave and have great enough momentum to be able to penetrate the contact surface and even the blast wave [7].

\subsubsection{Reaction Time Scale}

The metallic powders in an EBX do not react quickly enough to contribute to the detonation front [4], but they are able to contribute to the blast wave energy. This is possible because they react within the products of the condensed explosive. Perhaps more importantly, they react when they reach the shock-heated air. A schematic x-t diagram of an EBX explosion is shown in Figure 2.7. 


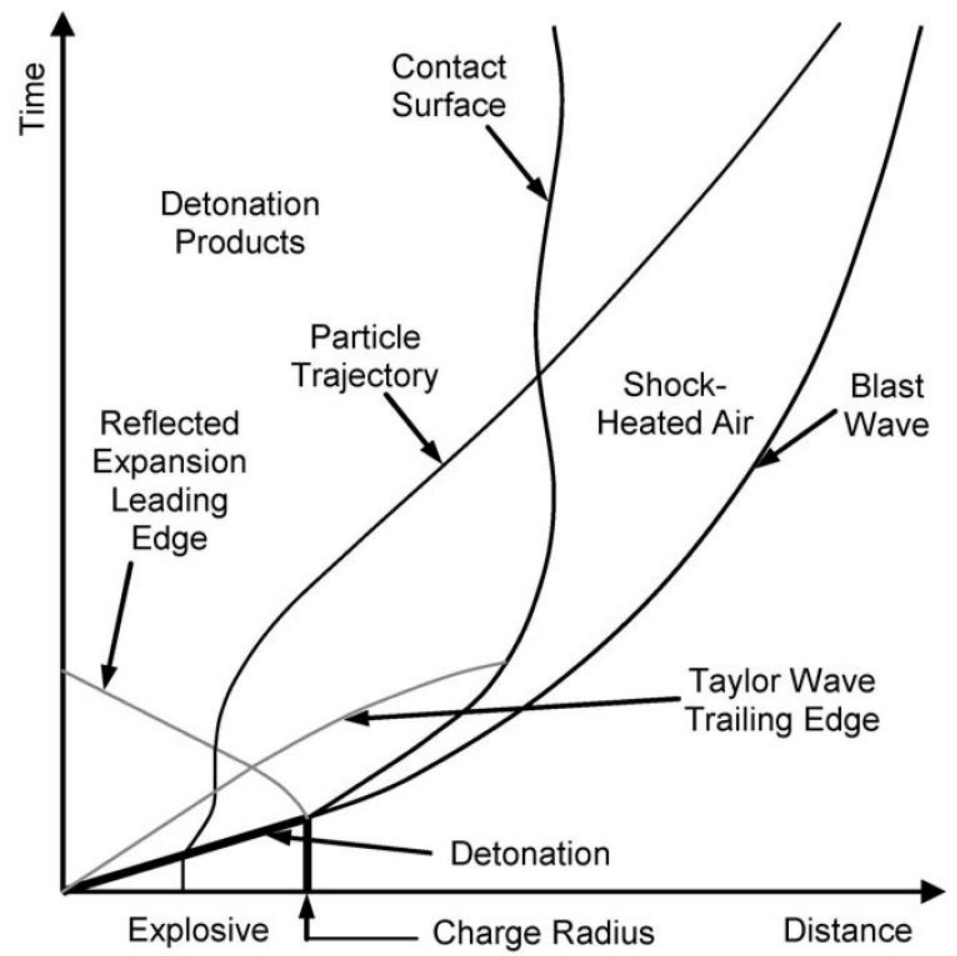

Figure 2.7: Schematic x-t diagram of detonation products flow field [4]

When the particles are within the detonation products (behind the contact surface), they react with the water contained within them. If they penetrate the contact surface, they react in the air that has been shock-heated by the blast wave. The Taylor wave is an expansion wave produced due to the spherical geometry of the charge.

The extra energy released from the powder has the ability to increase the overall effective energy of the explosion by 4-6 times if its release can be timed such that it contributes to the blast wave [4]. This contribution to the blast wave can be seen as a longer duration peak pressure in Figure 2.8. 


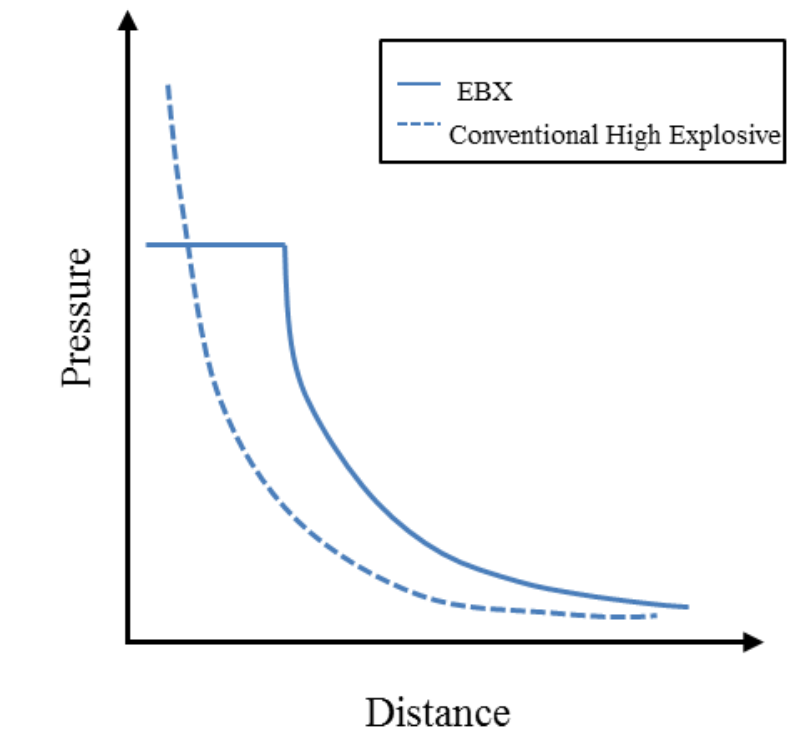

Figure 2.8: Comparison of pressure histories of conventional and EBX explosives

While conventional high explosives have a higher peak pressure than EBX devices, the longer duration peak pressure due to the contribution of the metallic powder to the blast wave creates a more energetic event. The area under the EBX curve in Figure 2.8 is larger than that of the conventional high explosive, showing the greater impulse of the EBX blast.

\subsubsection{Factors Affecting Particle Penetration of Shock}

Particle penetration through the shock front has been found to be dependent on the initial charge geometry. Due to the slow decay of the shock front, geometries that produced planar shock waves did not exhibit particle penetration. A diverging shock causes the front to slow rapidly, making penetration possible for a particle with sufficient momentum. The overtaking distance (defined as radius at which the particle crosses the shock front) has been shown to increase with an increased charge diameter. For smaller particle diameters, acceleration is more prone to being reduced by drag. This means that even if the particle does manage to penetrate the shock, the drag will decelerate the particle enough for the shock to pass it once again. The solid volume fraction of the 
particles has also been found to effect the overtaking distance. A reduction in the particle concentration causes less momentum loss from the gas in the charge detonation because there is less mass to accelerate. This gives the gas more momentum to accelerate fewer particles, resulting in a decrease in the overtaking distance [7].

\subsubsection{Critical Diameter}

It has been shown by Frost et al. [16] that a "prompt" reaction of the particle cloud will occur if the initial charge is of a critical diameter. A charge diameter less than the critical diameter will result in a "delayed" reaction. A delayed reaction is one that is of a timescale too long to contribute to the blast wave, typically being greater than $1 \mathrm{~ms}$. It was also determined that a charge of sub-critical diameter can become so small that it displays no reaction at all. The critical diameter for particle ignition (CDPI) was found to depend on particle size, shape, mass fraction and material [4]. Between the limits of prompt and delayed reactions, a transitional regime was found to occur where pockets of prompt reaction could be found within the particle cloud. The transitional blast strength was found to fall between the limits of the super and sub-critical diameter. The relationship between charge size and particle size is shown in Figure 2.9. 


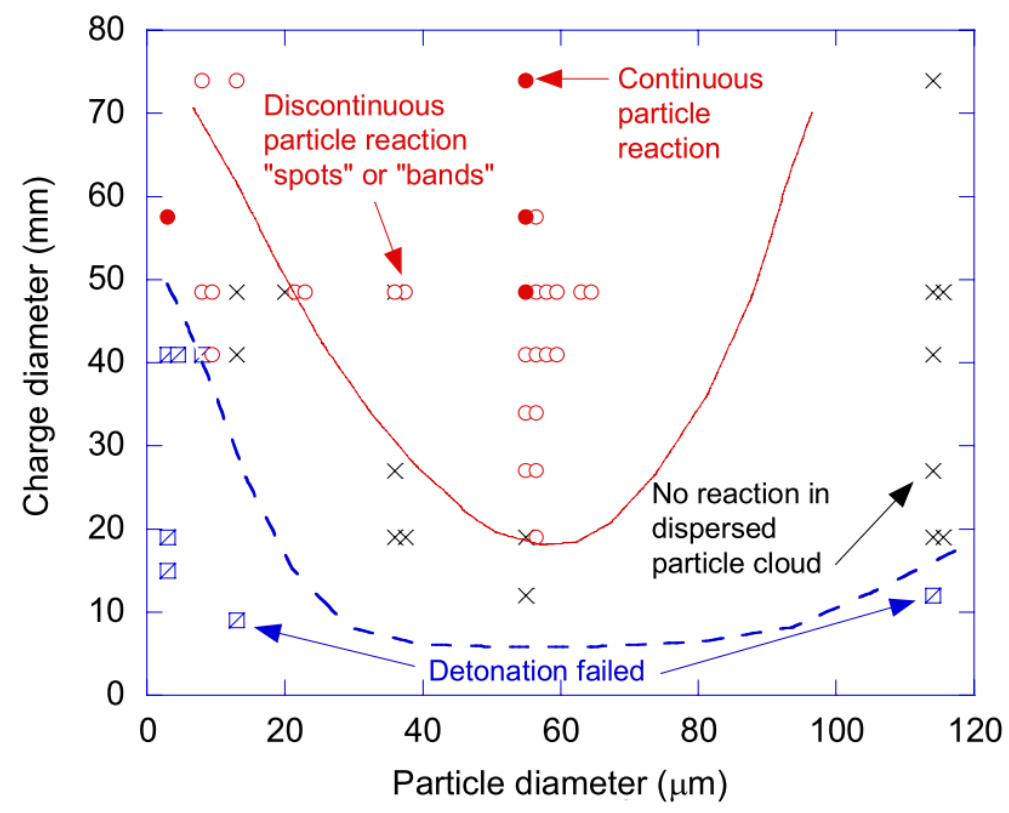

Figure 2.9: Critical charge diameter for particle ignition [16]

\subsubsection{Inert Particles}

From the above discussion, it is clear that the reactivity of metal particles is highly dependent on their initial dispersal. Because of this, it is essential to understand the early time dispersal before they react in the complex wave structure of an EBX. By looking into the dispersion of inert particles, fundamental insight can be gained about explosive particle dispersal.

Recent experiments by Frost at al. [17] were carried out using $4.2 \mathrm{~kg}$ of sand packed around $82 \mathrm{~g}$ of $\mathrm{C} 4$ plastic explosive. The detonation of the spherical charge resulted in a radial acceleration of the particles as shown in Figure 2.10. 


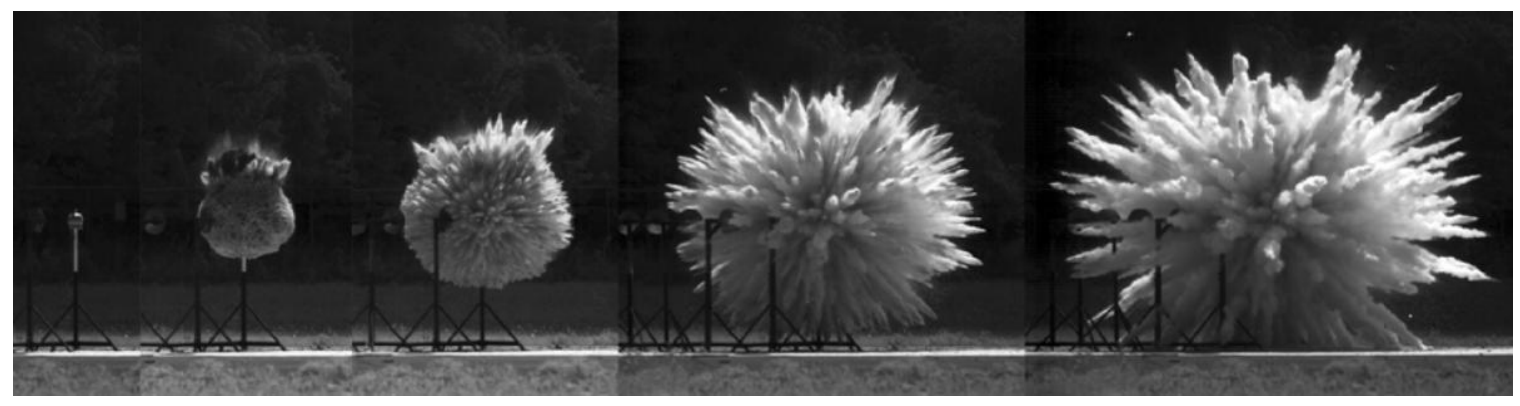

Figure 2.10: Explosive dispersal of packed sand particles. Inter-frame time is $2.5 \mathrm{~ms}$ between the first three frames and 5 ms between the last 3 frames [17]

The $0.5 \mathrm{~mm}$ diameter sand particles are distributed radially as the detonation wave reaches the particle bed surface. At this time, an expansion wave is reflected back into the particles and a spall layer forms at the particle bed surface. A density change is noticed in the particle bed surface at the time the reflected expansion arrives at the particle bed inner surface [18]. It was then observed that the particle bed breaks into fragments on the scale of the thickness of the initial bed, and produce a series of narrow, aerodynamically stable jets $[17,18]$. The stochastic nature of the material property, morphology, and bed configuration influence the development of jets, where the particle flow can remain as dense for a long time. The jets can influence particlegas mixing and blast wave propagation [18].

\subsubsection{Dispersion Flow Regimes}

The shock wave initially propagates through a granular particle bed during the detonation and once the particle cloud is dispersed the flow becomes a dilute gas-solid flow. Between these two regimes the flow can be described as a dense gas-solid flow [7]. The plot shown in Figure 2.11 shows when dense flow occurs in an explosive dispersal. 


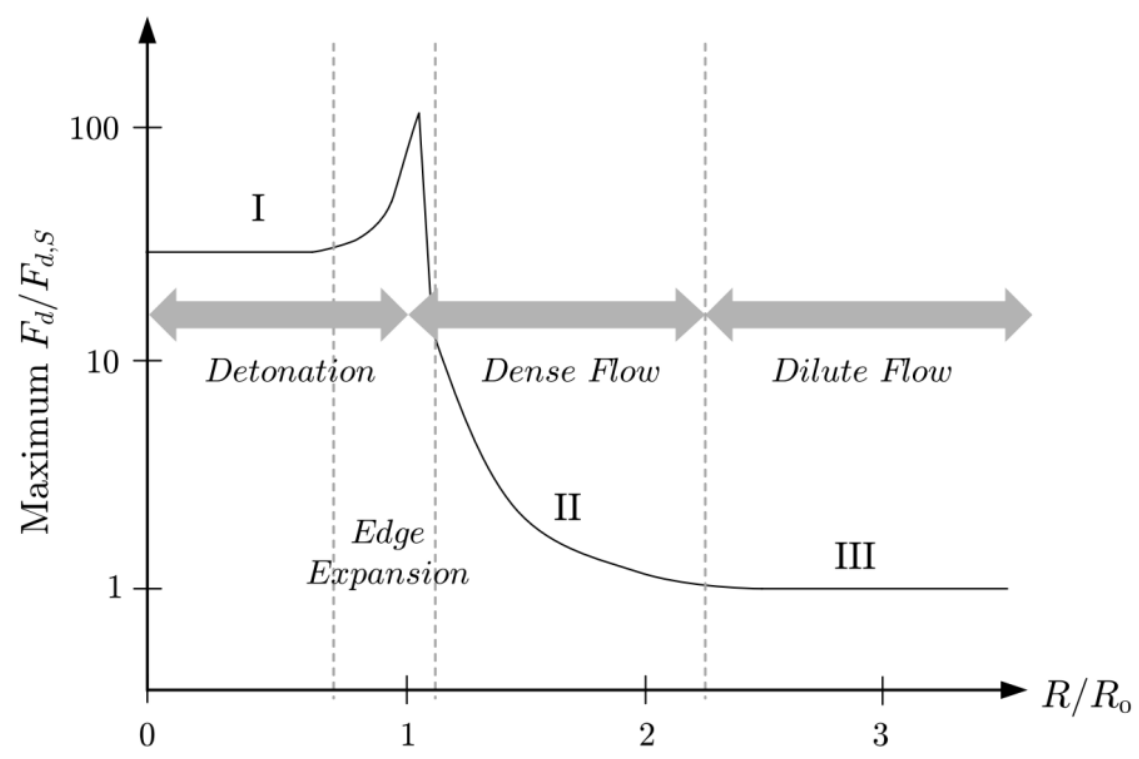

Figure 2.11: Dispersion flow regimes. The drag force acting on the particles, $F_{d}$, is normalized by the drag acting on a single sphere, $F_{d, S} . R_{0}$ is the charge radius [19]

After the initial particle movement caused by the detonation, dense flow occurs, where it can remain in the particle jets before transitioning to a dilute flow.

The solid volume fraction of the flow, $\varphi_{p}$, is what determines which of the three regimes the flow falls in. For a dilute gas-solid flow $\left(\varphi_{p}<0.01\right)$, it is assumed that the particles are very far apart and the probability of a collision between them is very low. For this reason, particle interactions can be ignored and the pressure and speed of sound are close to those of the gas phase. In the case of the granular flow $\left(\varphi_{p} \geq 0.5\right)$, the particles are assumed to be in direct contact with each other and since there is little gas in-between, the pressure and speed of sound are close to that of the solid phase. In the dense gas-solid regime, particles collide randomly and pressure and speed of sound have not been well established [7].

X-ray radiographs of a dispersal event are shown in Figure 2.12. This shows a glass bulb containing packed beds of steel particles. The movement of the cloud front and a gradient within it is clearly visible. 

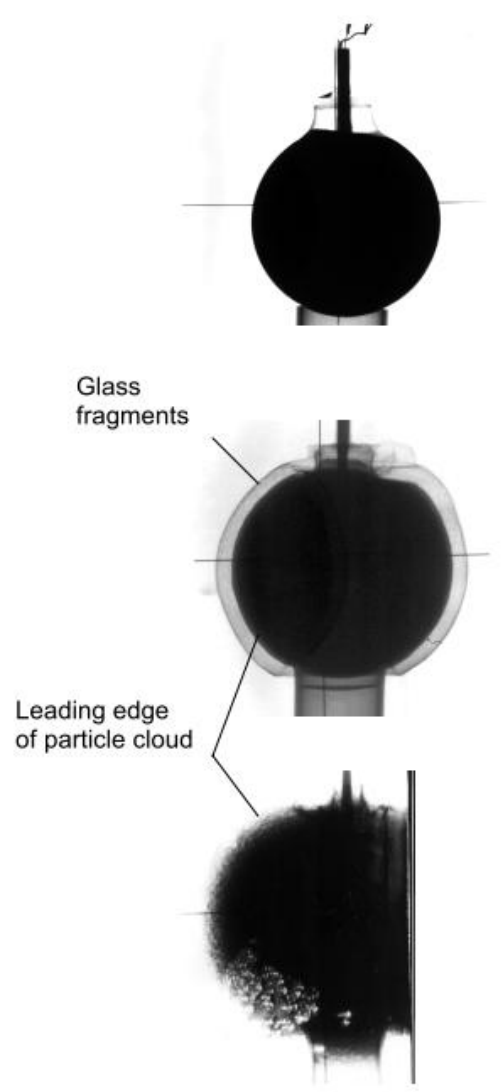

Figure 2.12: $X$-ray radiographs of a particle dispersal event. Images at $0 \mu \mathrm{s}, 43 \mu$ s and 102 $\mu \mathrm{s}[7]$

\subsection{Small-Scale Particle Dispersion}

While explosive dispersal can be studied on a large scale, it is difficult to extract detailed information of the physical phenomena that occur during the dispersal. In order to gain detailed information on specific aspects of the dispersal, controlled tests are required to study phase interactions between inert particles and the air. Zhang et al. [7] identified particle drag as the dominating force that dictated momentum transfer between phases. 


\subsubsection{Drag on a Single Particle}

In order to understand the particle acceleration in a multiphase flow, the drag forces acting on a single particle must first be examined. Any particle moving through a continuous fluid is subject to aerodynamic forces acting upon it, as shown in Figure 2.13.

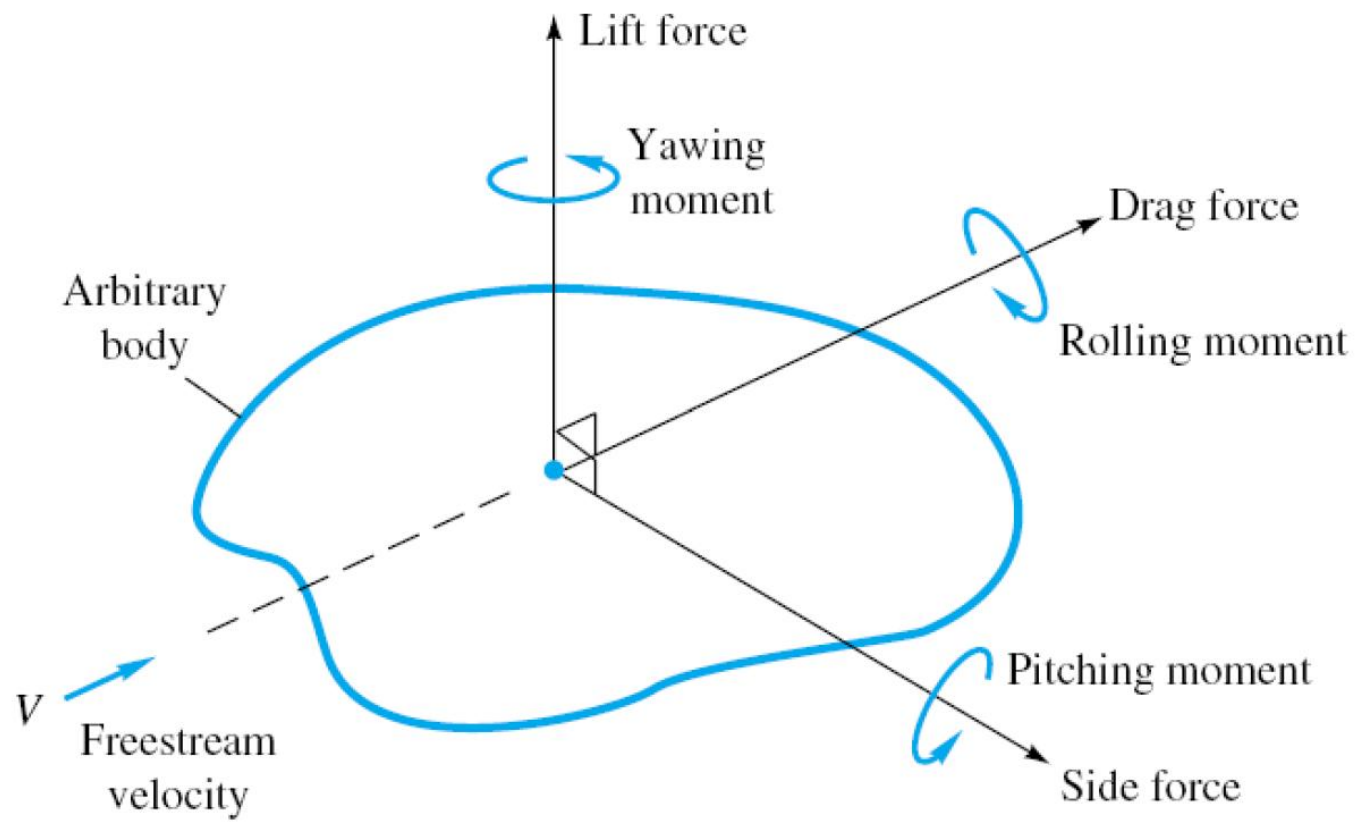

Figure 2.13: Forces acing on an immersed body [20]

For this discussion, the particle will be examined in one-dimension, neglecting lift and side forces and assuming a spherical particle. The drag force acting on the particle is in the direction opposite to the velocity vector of the moving particle and is dependent on several flow parameters. To obtain an expression for the drag force acting a on a single particle, the particle Reynolds number, $R e_{p}$, must be defined.

$$
R e_{p}=\frac{\rho_{f} u_{0} D_{p}}{\mu_{f}}
$$


The Reynolds number is a dimensionless ratio of inertial and viscous forces of the fluid surrounding the particle. It is based on fluid properties, namely the density, dynamic viscosity and free-stream velocity as well as a characteristic dimension. In the case of a spherical particle, the particle diameter is chosen as the length scale. For a small particle, the ratio of inertial to viscous forces is low, giving $R e_{p} \rightarrow 0$. As the Reynolds number gets smaller, the acceleration terms in the Navier-Stokes equations become negligible [20] for a spherical particle. This is referred to as a creeping flow, where it is assumed that the particle is a rigid sphere in an infinite medium with no slip at the surface [21]. In case of the present research, the particles are very small, with particle diameters being close to $100 \mu \mathrm{m}$, in a flow velocity of approximately $430 \mathrm{~m} / \mathrm{s}$. In an explosive dispersal event, higher particle velocities have been observed, still giving Reynolds numbers as low as on the order of $10^{-7}$.

To calculate the drag force in the creeping flow regime, the contribution comes from two different sources. The first is the pressure force acting perpendicular to the particle surface, and the second is the shear stress at the surface. Taking the Navier-Stokes equation and assuming steady flow, neglecting inertial terms and assuming a no-slip boundary condition, the expressions for the normal and tangential (or pressure and shear) drag forces can be derived (see Nedderman [22]) and are given by,

$$
\begin{aligned}
& F_{n}=2 \pi \mu R_{p} u \\
& F_{t}=4 \pi \mu R_{p} u
\end{aligned}
$$

A summation of these two drag components gives the total drag force acting on the sphere 


$$
F_{D}=F_{n}+F_{t}=3 \pi \mu D_{p} u
$$

This represents the total drag force acting on the sphere if gravity is neglected, and is known as Stokes' law. As previously mentioned in this section, it is assumed that for low Reynolds number flows, acceleration terms in the Navier-Stokes equations can be neglected, making Stokes' law only valid for the condition of $R e_{p} \ll 1$. At $R e_{p}=1$, the predicted drag force is $13 \%$ lower than it actually is due to the neglected terms [21] while it is within $2 \%$ accuracy for $\operatorname{Re}_{p}<0.1$ [23].

A drag coefficient, $C_{D}$, is used to non-dimensionalize the drag force and is based on the projected area of the sphere and the dynamic pressure.

$$
C_{D}=\frac{F_{D}}{\frac{1}{8} \pi D_{p}{ }^{2} \rho_{f} u^{2}}
$$

In the case of low Reynolds number Stokes flow, it is given by

$$
C_{D}=\frac{24}{R e_{p}}
$$

The above drag equations only apply for incompressible flow. If the flow velocity is high enough, the coefficient of drag becomes a function of the Mach number and Reynolds number. This is because, depending on the body shape, local velocities near the body can become sonic even while the stream Mach number is subsonic [20]. Bow shocks can form ahead of the body and increase the normal (pressure) drag force. For a sphere the critical Mach number, $M_{\text {crit }}$, at which compressibility affects the drag coefficient, is about 0.6 . General speaking, the drag based on the Mach number is unimportant for $M<0.6$, as Reynolds number based drag dominates. From $M=$ 0.6 to $M=1.0$, both Reynolds number and Mach number are important for determining the drag 
coefficient. At supersonic conditions Mach number based drag dominates. Predictions of drag coefficients based on Mach number come mainly from empirical data.

\subsubsection{Phase Interactions}

In the case of a multiphase flow, where there are many particles present, particle drag behavior is dependent on a variety of factors. Often the flow of solid particles and the flow of the gas are treated separately, and coupled through their interactions [7]. Each phase has its own volume fraction where,

$$
\varphi_{g}+\varphi_{s}=1
$$

Baer and Nunziato [24] developed a theory for granular flows where it was assumed that each phase behaves as a single material, except when there is an exchange of mass, momentum or energy between phases. It was also assumed that since each phase was treated separately, conservation equations remained the same, with a source term being added to couple the phases. A number density equation was included to account for breakup or agglomeration of particles and compaction of particles was also accounted for [24]. The summation of the conservation equations for each phase will produce the conservation equations of a single substance. The rate of change of the phase density, due to mass exchange by chemical reactions and expansion, is expressed in the mass conservation equation. The energy equation represents the phase specific internal energy taking into account work done by pressure, heat transfer due to conduction, external heat sources, inter-phase energy exchange, energy due to momentum transfer, and energy from the appearance/disappearance of a phase [24]. Finally, the conservation of momentum gives that the rate of change of momentum is related to pressure gradient, external body forces, momentum from the appearance/disappearance of a phase, and inter-phase momentum transfer. The phase momentum transfer can be represented as drag and is dominant over the other forces. Simulations have shown that standard drag models do not properly 
represent the momentum transfer seen in the dense flow that follows detonation [7]. This momentum exchange between phases is currently the biggest problem modelling multiphase flows, as a physical model in the dense gas-solid flow regime has not been fully established [25].

Studies in the dense flow regime have yielded drag coefficients that can differ greatly from those acting on a single particle. Based on equations developed by Smirnov [26], drag coefficients for a range of Reynolds number and particle volume fraction were plotted by Ripley [19], showing their deviation from single particle estimations. This is shown in Figure 2.14.

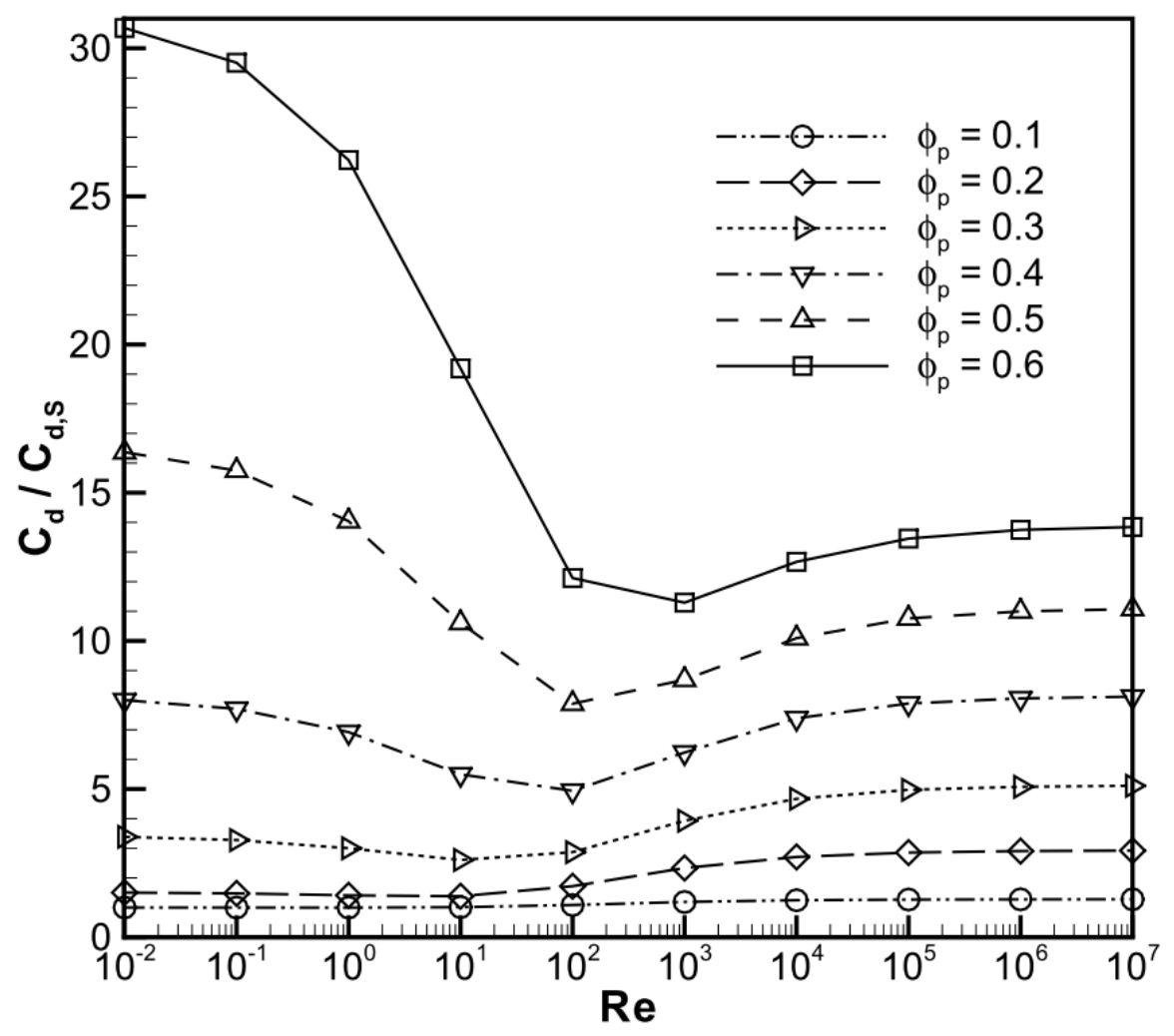

Figure 2.14: Deviation of drag coefficients in dense gas-solid flow regime from single particle drag coefficients. $C_{d}$ is the drag coefficient for a particle in a dense flow. $C_{d, s}$ is the drag coefficient for a single particle [19] 
At low Reynolds numbers and high particle volume fractions (i.e. dense gas-solid flow) it is clear that the physics of single particle drag cannot be applied to a dense gas-solid flow. This reveals that a very complex interaction between the two phases exists.

\subsubsection{Shock Tube Studies}

Numerous shock tube studies have been performed on multiphase mixtures, but few of them are in the dense gas-solid regime [9]. Using a $6 \mathrm{~m}$ long, $13 \mathrm{~cm}$ square cross-section vertical shock tube, Rogue et al. [9] performed a series of particle dispersal experiments with glass or nylon spheres with diameters of $1 \mathrm{~mm}, 1.5 \mathrm{~mm}$, and $2 \mathrm{~mm}$. Incident shock Mach numbers ranging from 1.1 to 1.5 were used. Particles were placed on a plastic membrane or metal grid and impacted by the shock wave. Arrangements of a single layer and a double layer of particles were tested using the plastic membrane, while a steel grid was used to test a thick particle bed. It was found that the incident shock reflected off the particle bed, travelling upstream, while a weak transmitted shock wave continued downstream. This resulted in the dispersal of the particles, as shown in Figure 2.15.

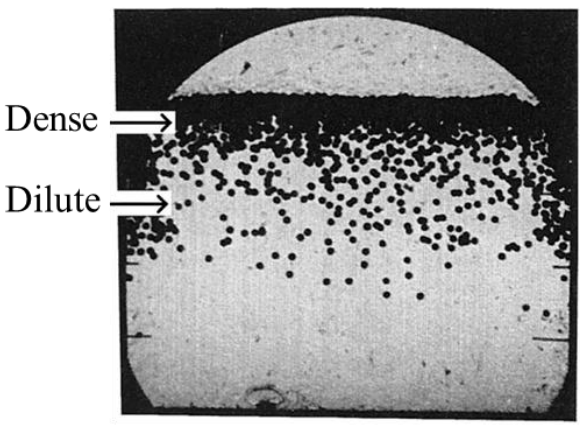

(a)

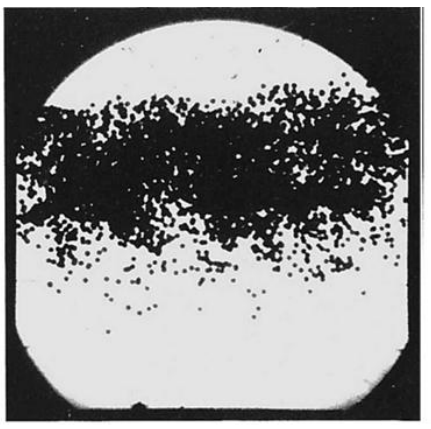

(b)

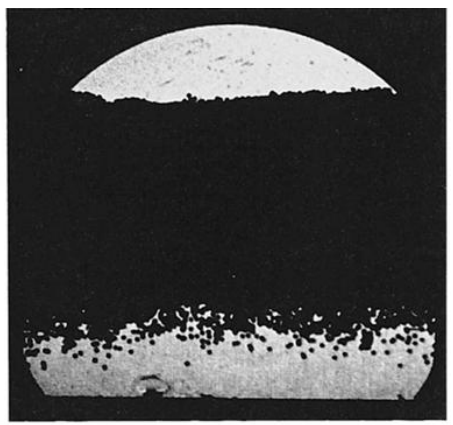

(c)

Figure 2.15: Shadowgraph images of dense gas-particle flow by a Mach 1.3 shock wave in air for a) a single layer of $2 \mathrm{~mm}$ glass particles at $2.65 \mathrm{~ms}$, b) a double layer of $2 \mathrm{~mm}$ diameter glass particles at $3.84 \mathrm{~ms}$, and c) a $2 \mathrm{~cm}$ thick bed of $1.5 \mathrm{~mm}$ diamter glass particles at $4.88 \mathrm{~ms}[9,19]$ 
In the case of the single layer (Figure 2.15a), the particles disperse with a dense leading edge and a dilute upstream cloud edge. For the double layer (Figure 2.15b), increased particle collisions cause the cloud be become more diluted then the single layer cloud [9]. The thick bed (Figure 2.15c) was found to remain impermeable to the high reflected pressure for much longer than the other configurations. The key finding was that the estimated drag coefficient was found to be higher in dense flow field than established dilute gas-solid flow relations predicted. It is important to note that this experiment did not test particle sizes typical of multiphase explosives and the supporting membrane most likely had an effect on the flow in the shock tube.

In a similar experiment to the work presented in this thesis, Wagner et al. [12] used a gravity-fed particle "curtain". The roughly $3 \mathrm{~mm}$ thick particle curtain, consisting of 100 micron sized soda lime particles, had an estimated particle volume fraction of $\varphi_{p}=0.15$, which is in the dense gas-solid flow regime. Schlieren photography was used to visualize the interaction of the particle curtain with Mach number 1.67 and 1.95 shocks, see Figure 2.16. The dispersion was characterized by tracking the downstream and upstream edges of the particle cloud. 

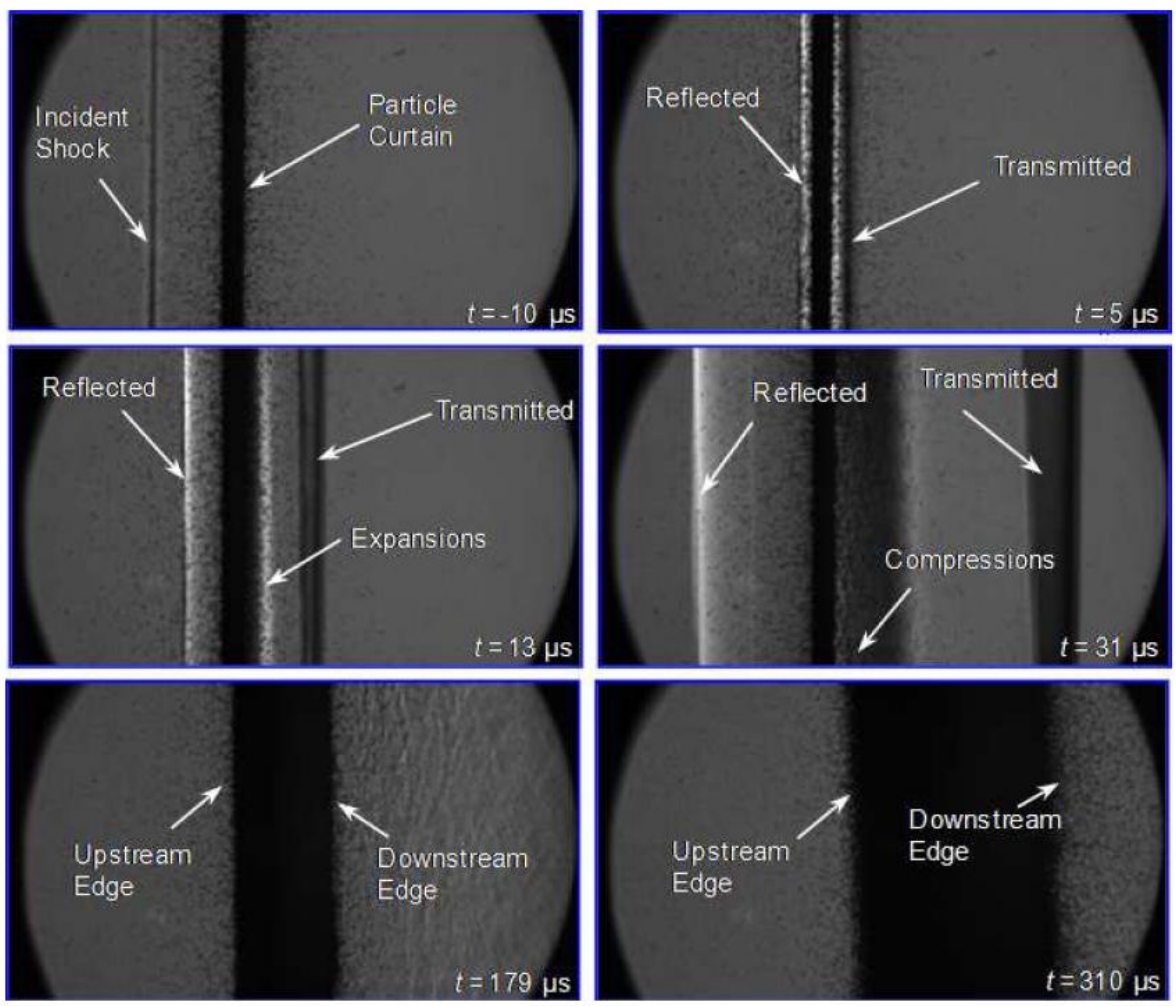

\section{Figure 2.16: Schlieren images of Mach 1.66 shock-particle curtain interaction [12]}

The images show that the interaction of the shock wave with the particle curtain produces a transmitted and reflected shock wave. Immediately following the shock interaction the particle cloud accelerates and grows axially, as the downstream edge moves faster than the upstream edge. The cloud dispersion was found to be exactly the same for both shock strengths tested. The particle curtain only extended $87 \%$ of the full $76 \mathrm{~mm}$ width of the square shock channel. As will be discussed later, the gap between the particle curtain and the shock channel glass side-wall is necessary to prevent sticking of the particles and the subsequent obscuring of the cloud upstream edge. The drawback is that the gaps on either side of the curtain introduce three-dimensional effects as the shock bypasses the particle curtain. For example, diffraction of the incident shock wave past the gap shows up as thickening of the shock wave in the schlieren image at $31 \mu \mathrm{s}$ in Figure 2.16 [12]. 


\section{Chapter 3}

\section{Experiment}

\subsection{Overview}

In this study, dense particle flow was generated by the interaction of a packed granular bed, initially in the form of a particle wafer, with a shock wave inside a tube. The initial packed granular bed is generated by compressing loose powder into a wafer with a particle volume fraction of $\varphi_{p}=0.48$ that is positioned inside the shock tube. This results in a clean experiment where no flow-obstructing supporting structures are present. Through high-speed shadowgraph imaging and the acquisition of pressure-time histories at different locations along the channel length, detailed information about the particle-shock interaction was obtained.

Using existing shock tube components, a control system was created along with data acquisition components to perform the experiments.

\subsection{Shock Tube}

The shock tube consists of a $1.96 \mathrm{~m}$ long, $10 \mathrm{~cm}$ diameter driver, a transition section, and a driven section consisting of five $0.6 \mathrm{~m}$ long, $7.6 \mathrm{~cm}$ square sections as shown in Figure 3.1. The transition section is $0.75 \mathrm{~m}$ long. Each section was machined using 6061-T6 aluminum. 


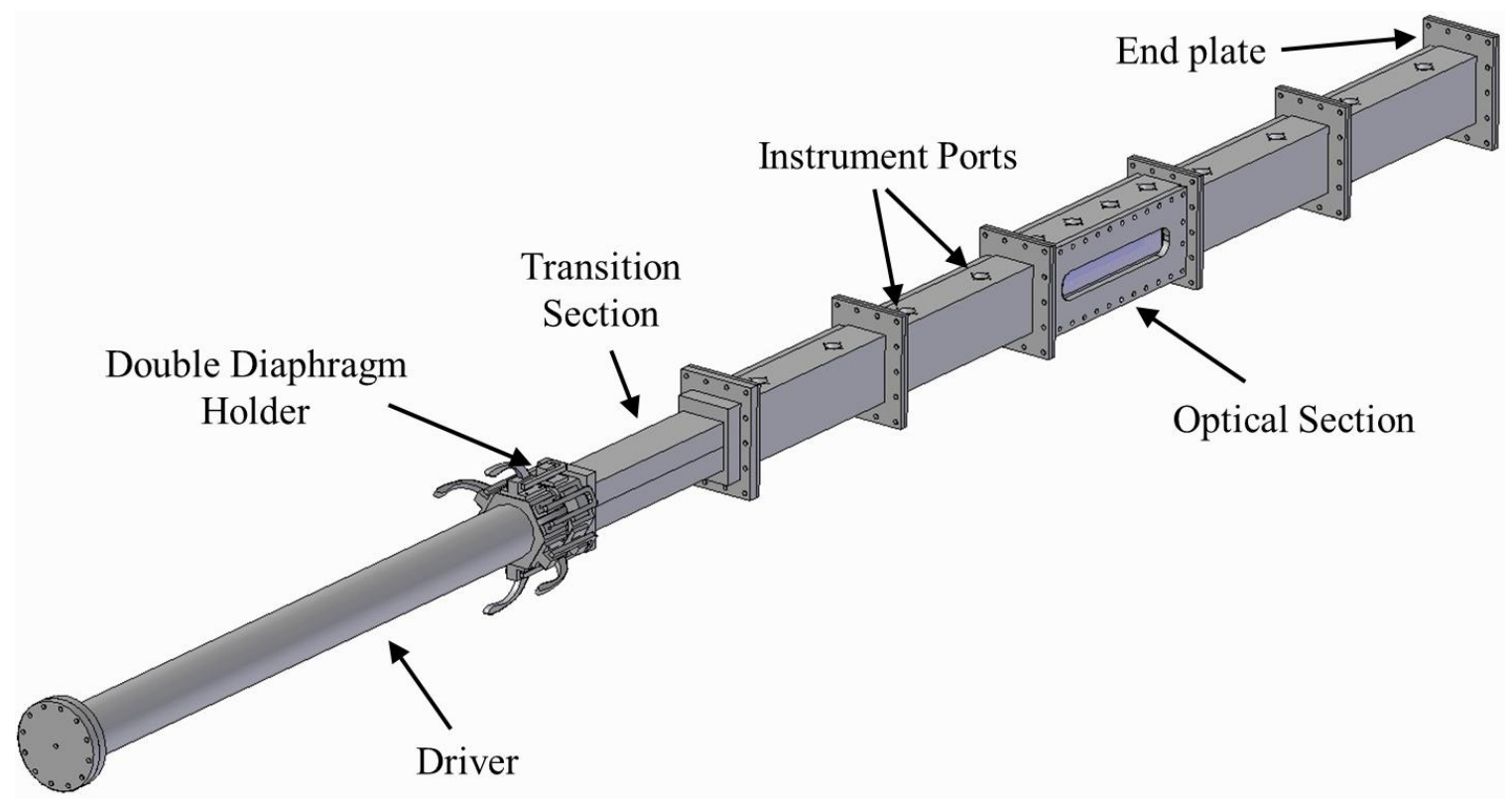

Figure 3.1: Experimental shock tube apparatus

For most of the testing, helium $\left(\mathrm{T}_{\mathrm{i}}=295 \mathrm{~K}\right.$ and $\mathrm{P}_{\mathrm{i}}=10$ bar $)$ was used in the driver and atmospheric air $\left(\mathrm{T}_{\mathrm{i}}=295 \mathrm{~K}\right.$ and $\mathrm{P}_{\mathrm{i}}=1$ bar $)$ was used in the driven section. All flanges and the double diaphragm holder were sealed with synthetic rubber Buna-N O-rings.

\subsubsection{Driver}

The $1.91 \mathrm{~cm}$ thick driver tube was designed to go up to a maximum driver pressure of $100 \mathrm{bar}$. Six clamps on the end allow for easy connection to the double diaphragm holder and the transition section. An Edwards RV3 vacuum pump was used to evacuate the driver, double diaphragm holder, and the plumbing system prior to filling with helium.

\subsubsection{Double Diaphragm}

Located between the driver and transition section was the double diaphragm holder. This $10 \mathrm{~cm}$ diameter aluminum ring holds one diaphragm on each side. The $81.1 \mathrm{~mL}$ volume between the two diaphragms was essential for creating reproducible incident shockwaves. A double diaphragm 
made of Mylar plastic is used to precisely control the driver pressure at the time of diaphragm rupture. An image of the double diaphragm holder is shown in Figure 3.2.

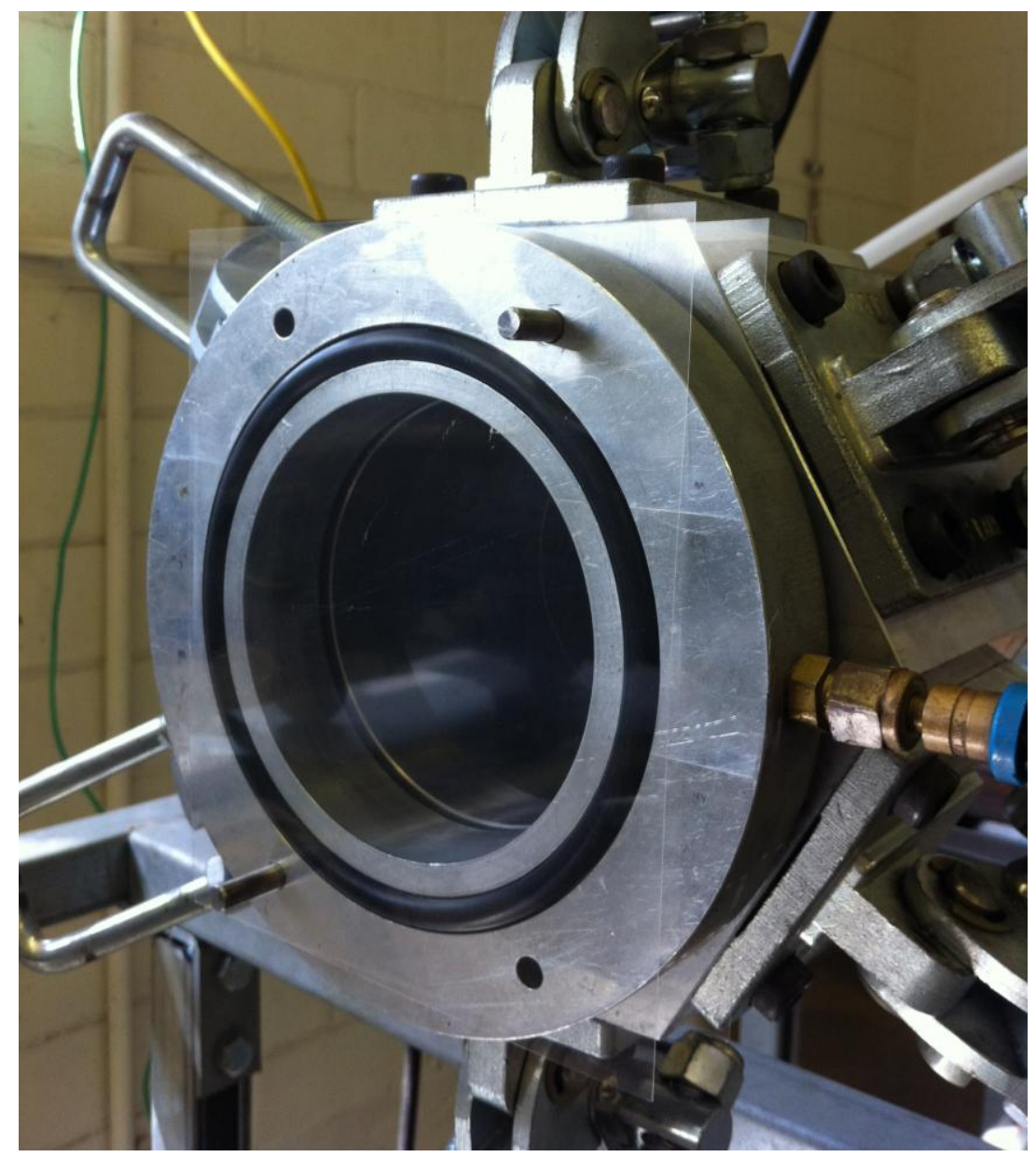

Figure 3.2: Double diaphragm holder in place at end of driver with Mylar diaphragms

The volume between the two diaphragms is filled to an intermediate pressure, between the driver and driven section initial pressures. When this volume is evacuated, the pressure differential becomes too great and the diaphragms burst. After performing a series of calibration tests, the burst pressure for a single diaphragm was found to be 4.46 bar. By varying the number of diaphragms in each of position of the double diaphragm configuration, varying driver pressures 
were achievable, giving the ability to produce any shock Mach number to a maximum of Mach 2. Testing with stronger shock waves is not possible in the shock tube due to the limiting strength of the glass windows.

For the majority of tests, a Mach 2 incident shock wave was used. From shock tube relations, it was found that a Mach 2 incident shock wave required a driver pressure of 11.00 bar using helium as the driver gas. The initial conditions used in most of the tests are given in Figure 3.3. Therefore the double-diaphragm configuration had to be set up such that no diaphragm would burst during filling, while still being able to fire the shock tube when at its final driver pressure. In order to do this, a two stage filling process was adopted. Since it was found that a single Mylar diaphragm could withstand a pressure differential of 4.45 bar, two of these diaphragms were placed on top of each other on each side of the diaphragm holder. Calibration tests revealed that the single diaphragms scaled very well, with two diaphragms on top of each other being able to withstand a pressure differential of 8.90 bar.

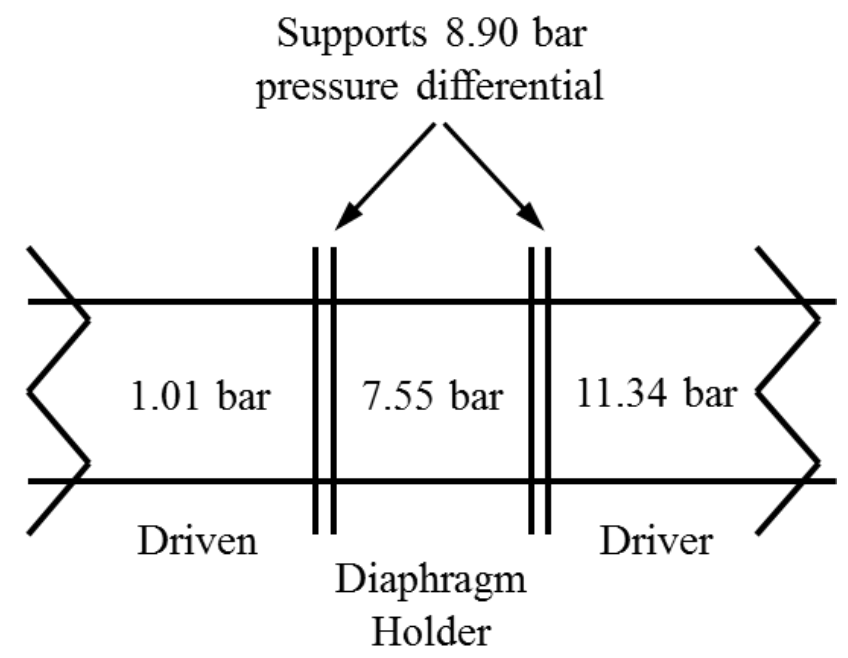

Figure 3.3: Diaphragm setup and fill pressures for Mach 2.0 incident shock wave 
A final driver pressure of 11.34 bar was used to account for leakage across valves. The 7.55 bar diaphragm holder pressure was chosen as a safe intermediate pressure that would not cause either of the diaphragms to burst prematurely.

Calibration tests were performed with no particle wafer in the driven section to ensure a planar shock wave of repeatable strength could be achieved. Figure 3.4 shows the incident shock wave from one of these tests.

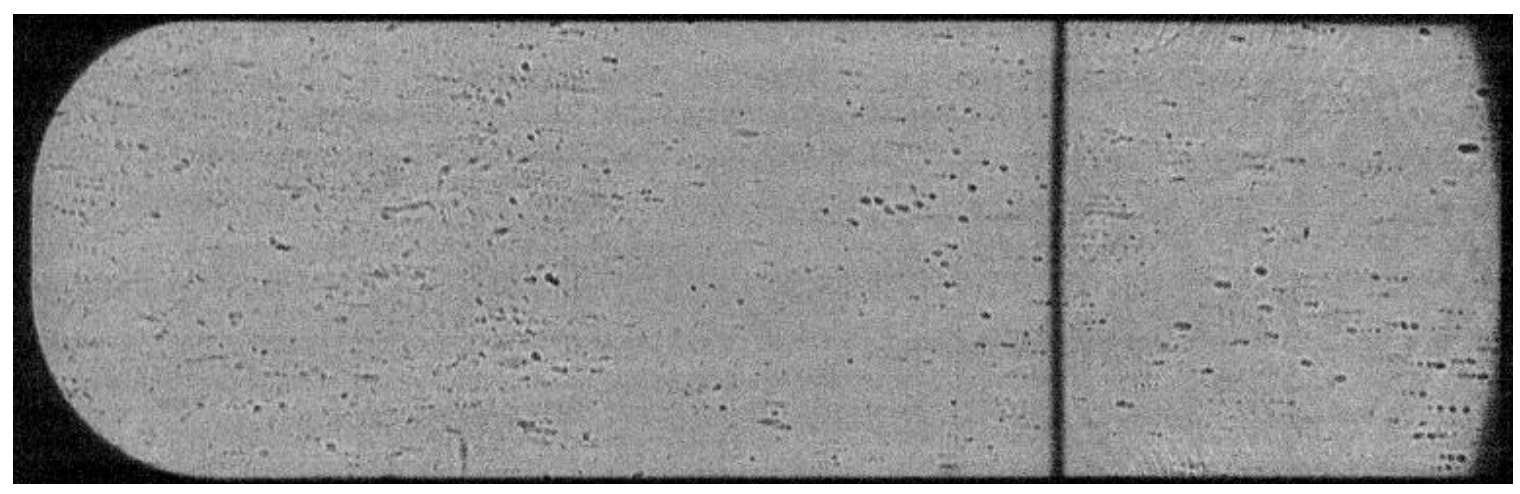

Figure 3.4: Calibration test showing planar incident shock wave propagating from right to left

Achieving a planar incident shock wave was important because any transverse waves would interfere with the dispersion process and a shock of repeatable strength was required to be able to compare tests.

\subsubsection{Transition Section}

The shock tube transition section allows the use of a round cross-section driver, with the square cross-section driven section. A smooth transition geometry was created through the use of a welded clamshell design, as seen in Figure 3.5. 


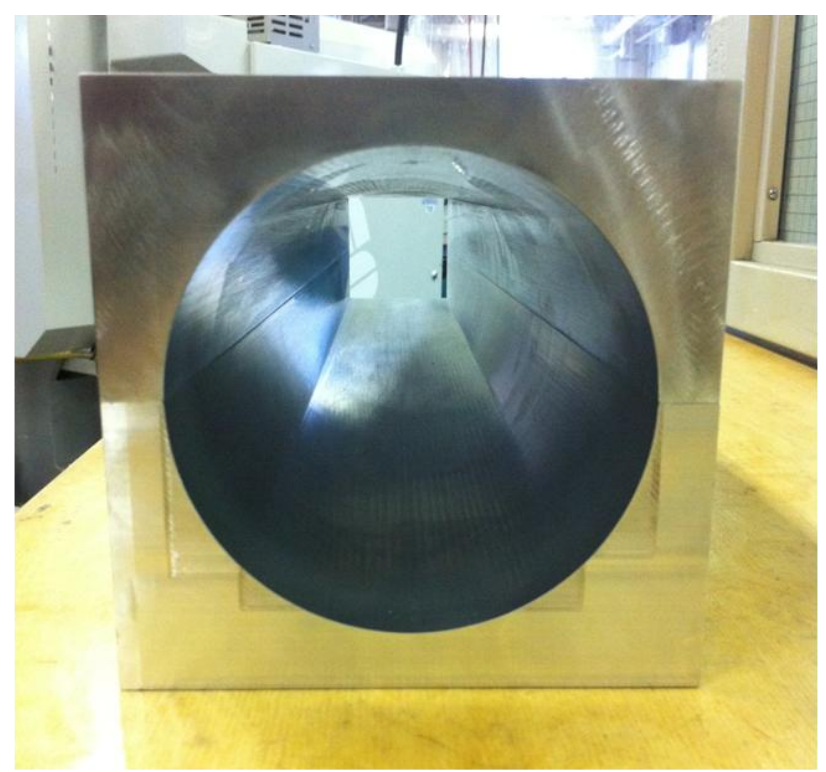

(a)
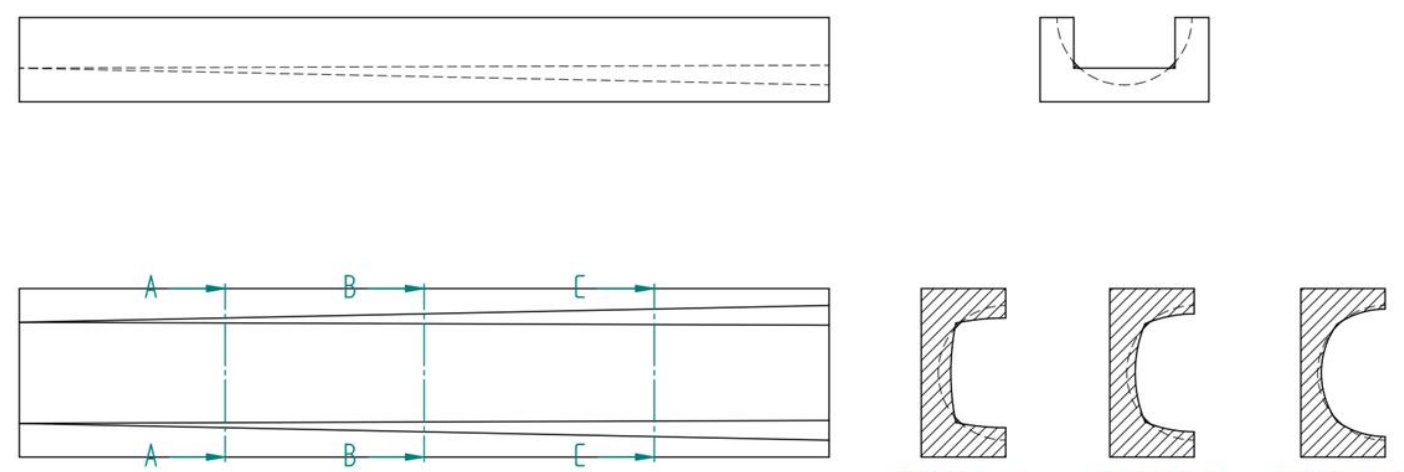

(b)

Figure 3.5: Round to square shock tube transition section a) prior to welding and b) CAD drawing showing gradual cross-sectional change

The transition section allowed for a geometry change while still maintaining a planar incident shockwave. It was advantageous to use a driver with a round cross-section because it is stronger and can be filled to a higher pressure than one with any other cross-sectional shape. The square 
cross-section was required in the driven section to accommodate the windows in the optical section.

\subsubsection{Driven Section}

One of the driven section channel segments was equipped with $1.91 \mathrm{~cm}$ thick glass windows for flow visualization. Tests were run with acrylic windows as well, but the hardness of the aluminum oxide particles used quickly abraded them to the point where high-speed visualization became difficult.

For data acquisition, six piezoelectric pressure transducers (PCB model 113A24) are flush-mounted on the top surface of the driven section. These transducers have a sensitivity of 5 $\mathrm{mV} / \mathrm{psi}$ with a rise time of $1 \mu \mathrm{s}$. A schematic is provided in Figure 3.6 that shows the location of the pressure transducers in the shock tube. Their exact locations downstream from the diaphragm are given in Table 3.1 .

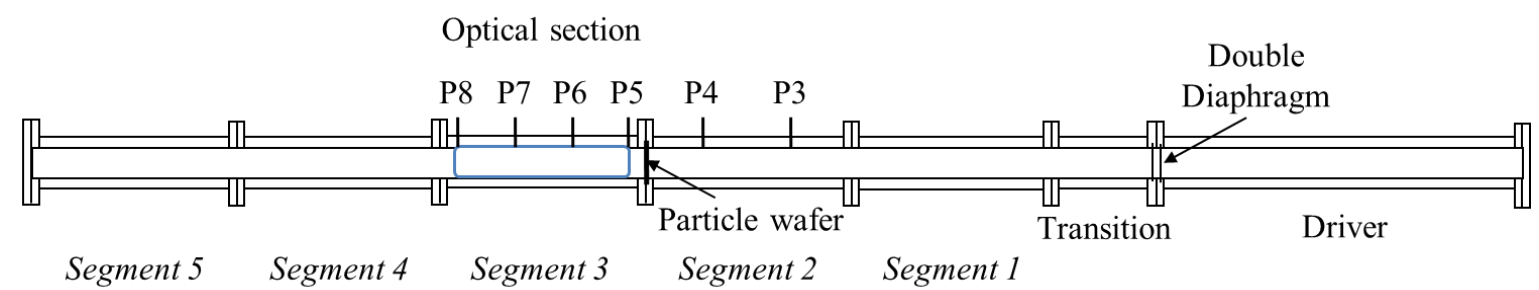

Figure 3.6: Schematic of shock tube showing the positions of the wafer, optical section, and the pressure transducers

Table 3.1: Downstream location of pressure transducers

\begin{tabular}{|c|c|c|c|c|c|c|}
\hline & P3 & P4 & P5 & P6 & P7 & P8 \\
\hline Distance from Diaphragm $(\mathrm{m})$ & 1.51 & 1.81 & 2.05 & 2.20 & 2.36 & 2.51 \\
\hline
\end{tabular}


For data acquisition, National Instruments LabVIEW 10 was utilized in conjunction with a NI PCI-6133 DAQ card and a NI BNC-2110 terminal block. The PCI-6133 contained 8 simultaneously sampling analogue inputs at a resolution of 14 bits and a maximum sampling rate of $3 \mathrm{MHz}$. Input range was specified as $0 \mathrm{~V}$ to $5 \mathrm{~V}$ for the pressure transducers to maximize accuracy. All tests were run at a sampling frequency of $1 \mathrm{MHz}$ and a resolution of 14 bits. The camera and data acquisition were triggered when the incident shock wave reached location P3. Once a signal was detected at P3 (from the passing shock wave), a $5 \mathrm{~V}$ pulse was sent to the TTL input on the camera to trigger video recording.

To test the effects of a circular cross-section, a $7.6 \mathrm{~cm}$ outer-diameter, $7.0 \mathrm{~cm}$ innerdiameter clear acrylic tube was inserted in segments 2 and 3 (see Figure 3.6) of the driven section. Gaps between the tube and the square cross-section were filled using an automotive body filler and sanded flat.

The shock tube also had the ability to utilize a low pressure driven section to achieve higher shock Mach numbers. In this scenario, the vacuum pump could be connected to the driven section to reduce the pressure to below atmospheric.

\subsubsection{End Plate}

For the majority of tests, a $1.27 \mathrm{~cm}$ thick aluminum end plate was utilized. To visualize the particle cloud from a head-on perspective, an endplate was constructed to allow for optical access. It consisted of a $2.54 \mathrm{~cm}$ thick acrylic window, sandwiched between two aluminum plates. A protective film was used on the inside of the acrylic window to prevent damage from the abrasive particle cloud. The end plate is shown in Figure 3.7. 


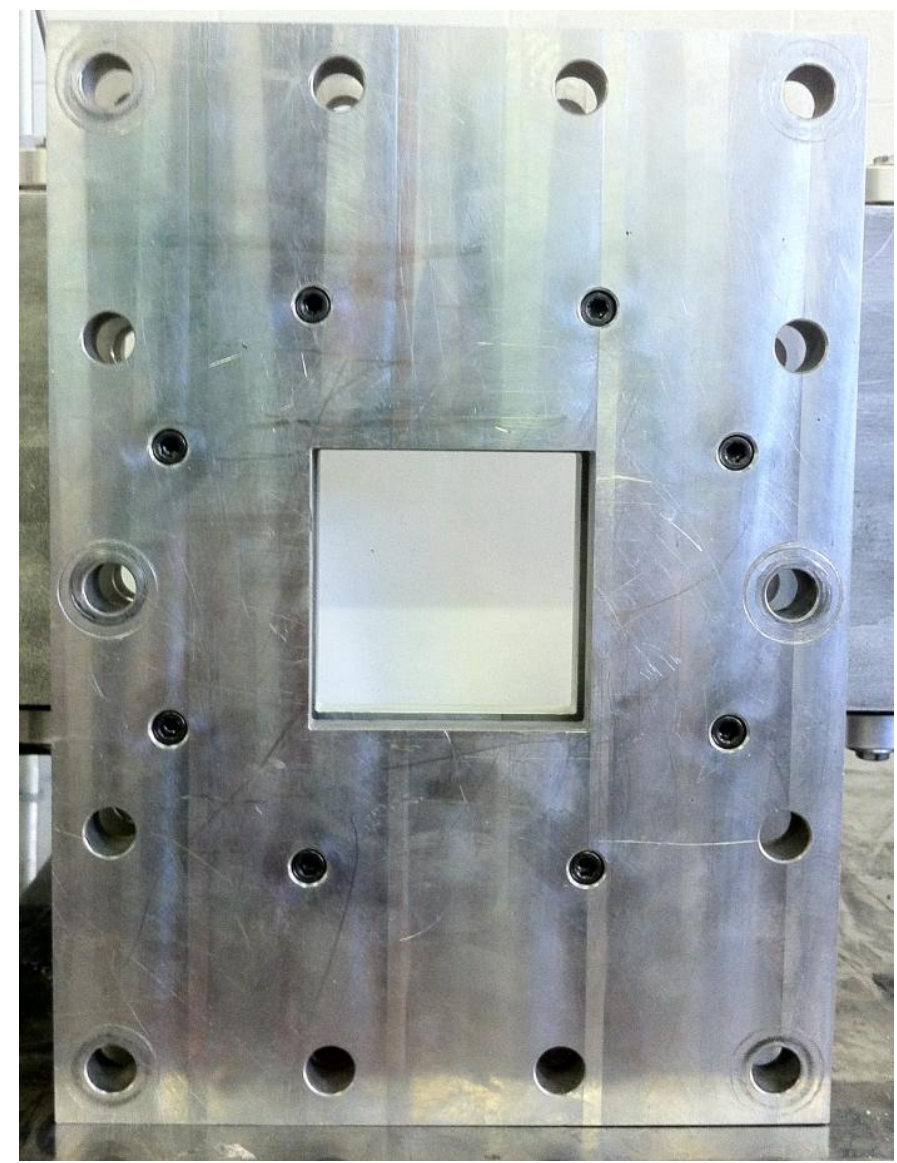

Figure 3.7: Shock tube end plate enabling optical access from head-on perspective

\subsubsection{Control Panel}

In order to operate the shock tube, a control panel was built containing pressure gauges and valves. A schematic of the control panel is given in Figure 3.8. 


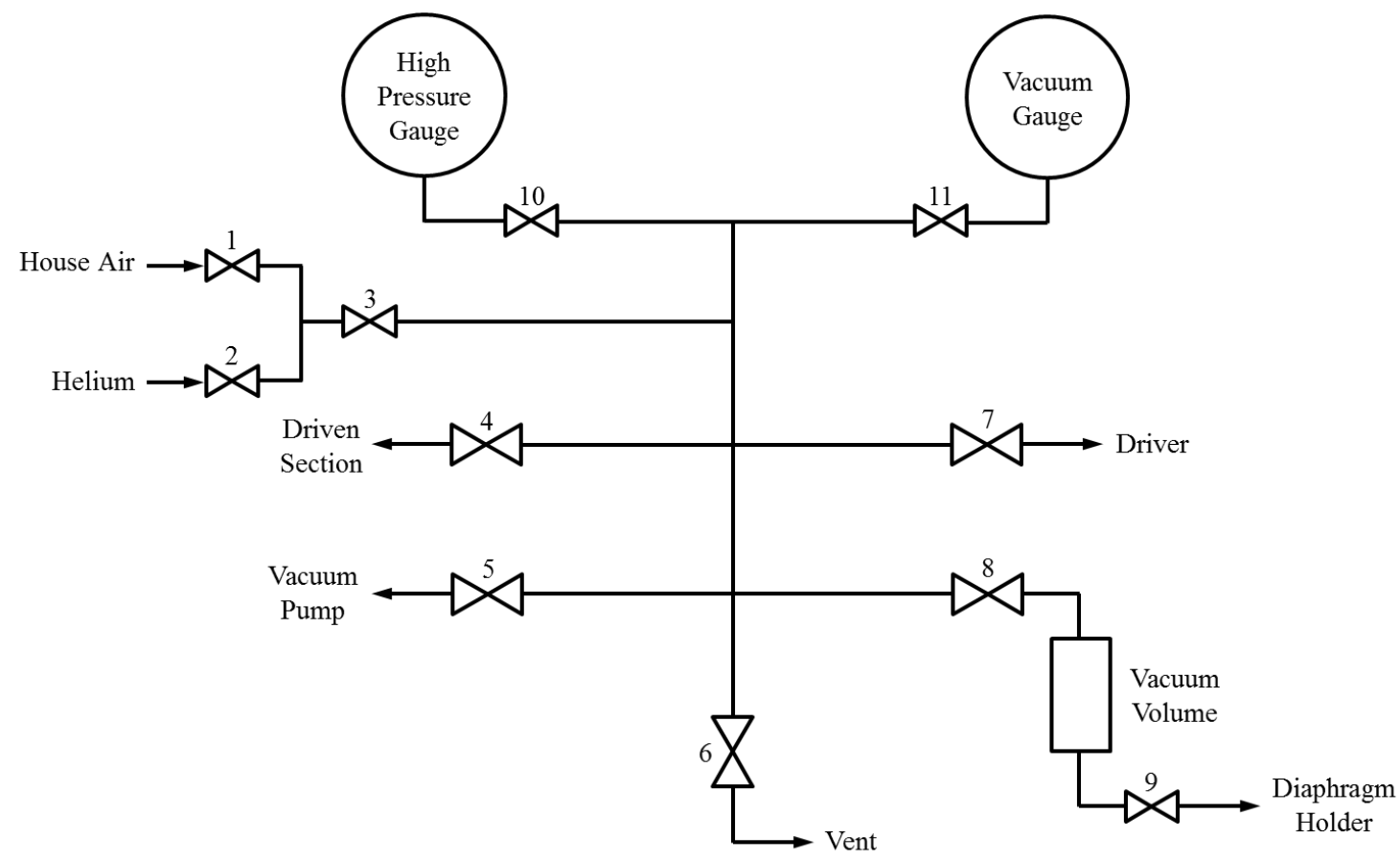

Figure 3.8: Schematic of shock tube control panel

The control panel was constructed using 0.25 in and 0.5 in stainless steel tubing with Swagelok valves and pipe fittings. Two needle valves (model 1RS4), numbers 1 and 2 in Figure 3.8, were used to precisely control the flow of air and helium respectively with valve 3 (model 43S4) acting as a stop. Valves 4 through 8 (model $45 \mathrm{~S} 8$ ) on the panel were ball valves used to allow or block flow from certain components of the shock tube, while valves 10 and 11 (model 43S4) were used to protect the pressure gauges (Omega model PGT) at the time of shock tube firing. The vacuum gauge had a pressure range of $30 \mathrm{mmHg}$ to $100 \mathrm{psi}$ and the high pressure gauge had a range of 0 psi to 600 psi. Valve 9 (model 43S4) was used to fire the shock tube. Helium driver gas was supplied by Praxair in compressed gas cylinders except for the "house air" that was supplied by a compressor. A detailed discussion of the shock tube operating procedure is given in Section 3.7. 


\subsection{Particle Wafer}

The particles are initially configured in a wafer that is located between P4 and P5, as seen in Figure 3.6, $1.97 \mathrm{~m}$ downstream of the diaphragm and $8.5 \mathrm{~cm}$ upstream of the edge of the optical section window. The particle wafer is created by compressing $70 \mathrm{~g}$ of aluminum oxide $\left(\mathrm{Al}_{2} \mathrm{O}_{3}\right)$ powder into a $7.6 \mathrm{~cm}$ square cavity machined into a $6.4 \mathrm{~mm}$ thick aluminum plate. The aluminum oxide has a density of $3960 \mathrm{~kg} / \mathrm{m}^{3}$ and is compressed using a 75 ton hydraulic press that compresses the powder into the plate cavity using the die shown schematically in Figure 3.9a. A photograph showing the compressed wafer in the plate cavity is shown in Figure 3.9b.

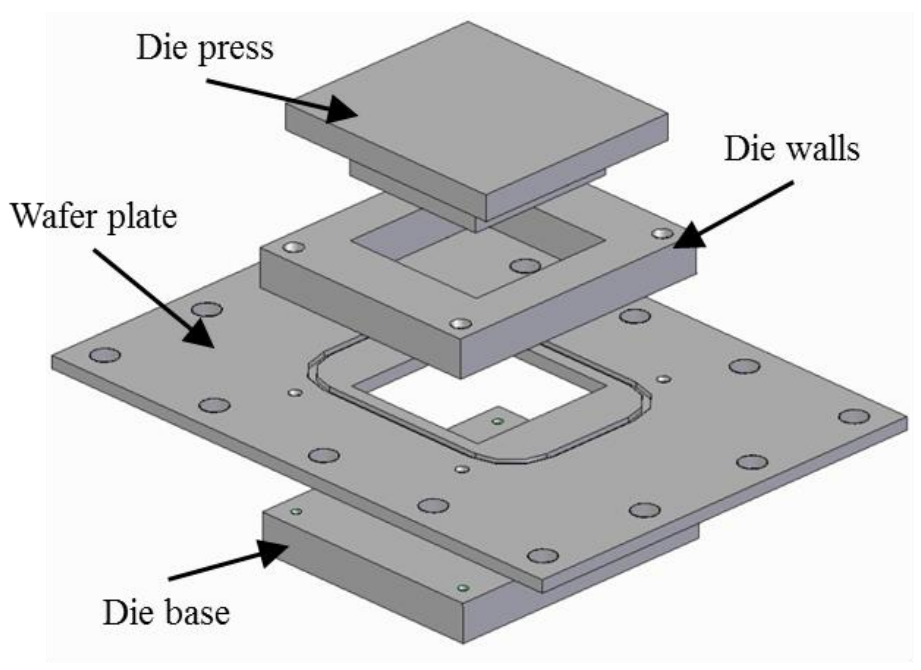

(a)

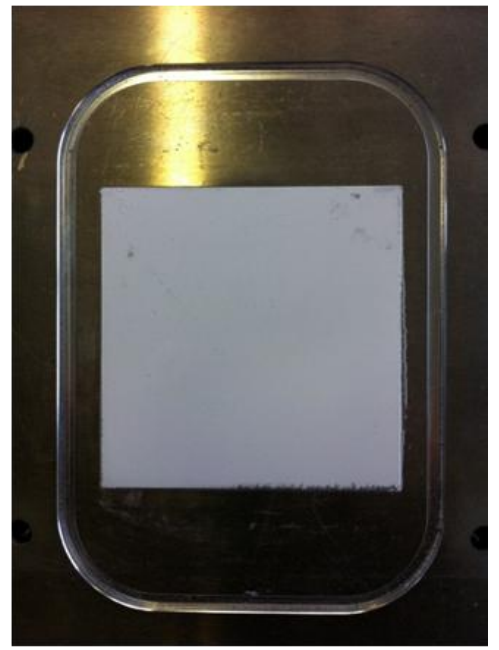

(b)

Figure 3.9: a) Die used to compress powder into the plate cavity b) Particle wafer loaded in the plate

As shown in Figure 3.6, the plate containing the wafer is sandwiched between two flanges such that the cavity lines up with the channel cross-section. This ensures that the particle wafer uniformly fills the entire shock tube cross-section such that no air bypass is possible following shock reflection. The wafer has a particle volume fraction $\varphi_{p}=0.48$ and the aluminum oxide 
powder has a mean particle diameter of $100 \mu \mathrm{m}$. Wafers of other densities were also created, having particle volume fraction of $\varphi_{p}=0.38$ and $\varphi_{p}=0.58$.

The initial position of the wafer between flanges (see Figure 3.6) makes it impossible to capture the initial shock and particle wafer interaction using the shadowgraph video system. Attempts to slide the wafer from the cavity into the optical section field-of-view near P5 resulted in unacceptable gaps forming between the wafer and the inner-channel walls. To do this a wafer was constructed using a different die method in which the bare wafer could be extracted from the die.

For a limited number of tests with the wafer initially in the optical section, a device was built for the square wafer to be pushed into the round cross-section with no gaps along the edges. A picture of the pushing device is shown in Figure 3.10.

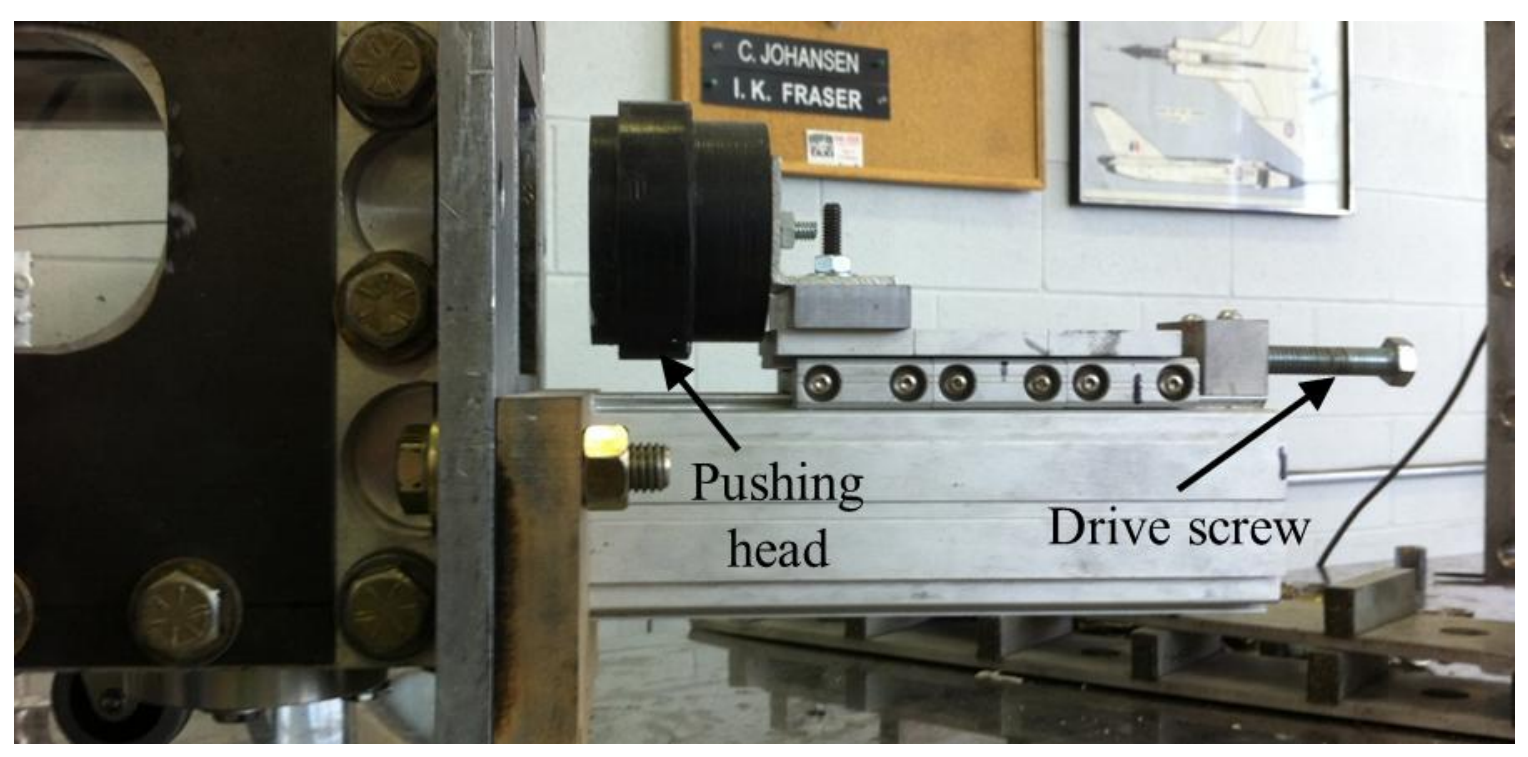

Figure 3.10: Particle wafer pushing device

To push the wafer inside the round tube, the particle wafer was first removed from the square die plate and placed in front of the black pushing head, as seen in Figure 3.10. The pushing head had 
a diameter of $7.0 \mathrm{~cm}$, which matched the inner diameter of the round tube. This ensured that the particle wafer pushed into the tube occupied the entire cross-section. To push the wafer, the drive screw was rotated, advancing the pushing head. The portion of the wafer outside of the pushing head face was sheared off as the square-shaped wafer entered the round tube. The drive screw was advanced until the rear of the wafer was flush with the optical section flange. An image of the wafer pushed into the round cross-section is shown in Figure 3.11. The grey material around the wafer is the material used to fill the area between the round tube and the square channel.

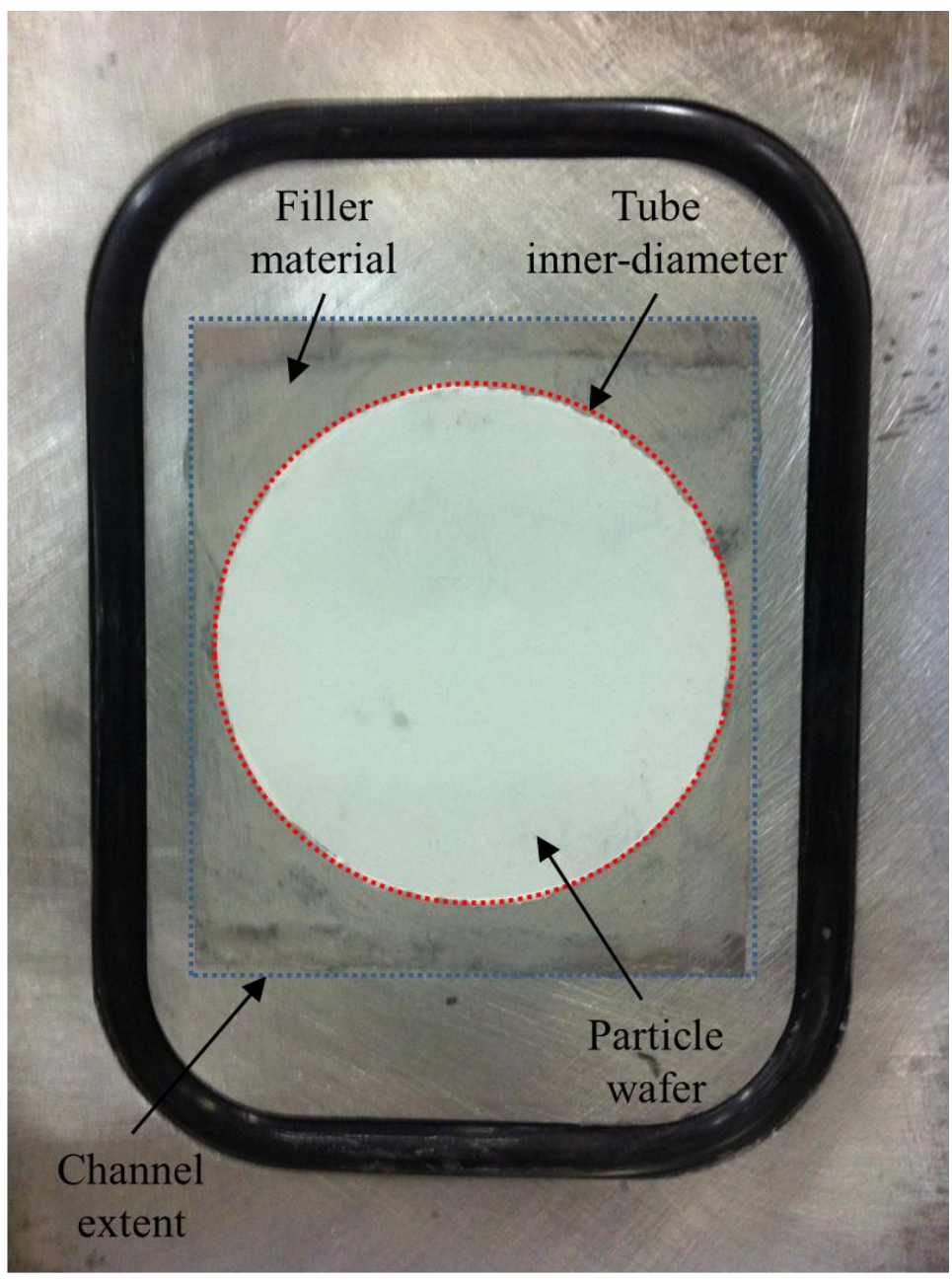

Figure 3.11: Wafer pushed into round cross-section 


\subsection{High-speed Photography}

To capture the details of the dispersion process in a way that could be analyzed, high-speed photograph had to be used. Tests in the square cross-section utilized a shadowgraph setup while round cross-section tests used a direct light setup. For each camera angle, a balance had to be struck with the shutter speed. A fast shutter was required so that the image was not blurred, but the shutter speed also had to be long enough to let enough light into the camera to sufficiently illuminate the image. Shutter speeds used were in a range of $2.5 \mu$ s to $1 \mu$ s. Three different camera angles were utilized: a side view, $30^{\circ}$ angle to the shock tube axis, and a head-on view. The camera field-of-view covers half the length of the optical section window shown in Figure 3.6 for side-view videos.

\subsubsection{Shadowgraph}

A single-pass shadowgraph system is used in connection with a Photron SA5 high-speed video camera to record the shock trajectory and the particle cloud movement. The Photron SA5 is a specialized high-speed camera capable of recording up to $1000000 \mathrm{fps}$ with a 369 ns shutter. The high light sensitivity (ISO 10000 equivalent) of the camera's complimentary metal-oxide semiconductor (CMOS) image sensor makes it ideal for high-speed imaging where high frame rates limit the light reaching the sensor. The camera records 12-bit uncompressed data directly to 8 GB

of random access memory (RAM) and is transferred to a computer over a gigabit Ethernet interface. The Photron FASTCAM Viewer 3 software allows for the control of the camera resolution, frame rate and shutter speed. A schematic of the camera setup is shown in Figure 3.12. 


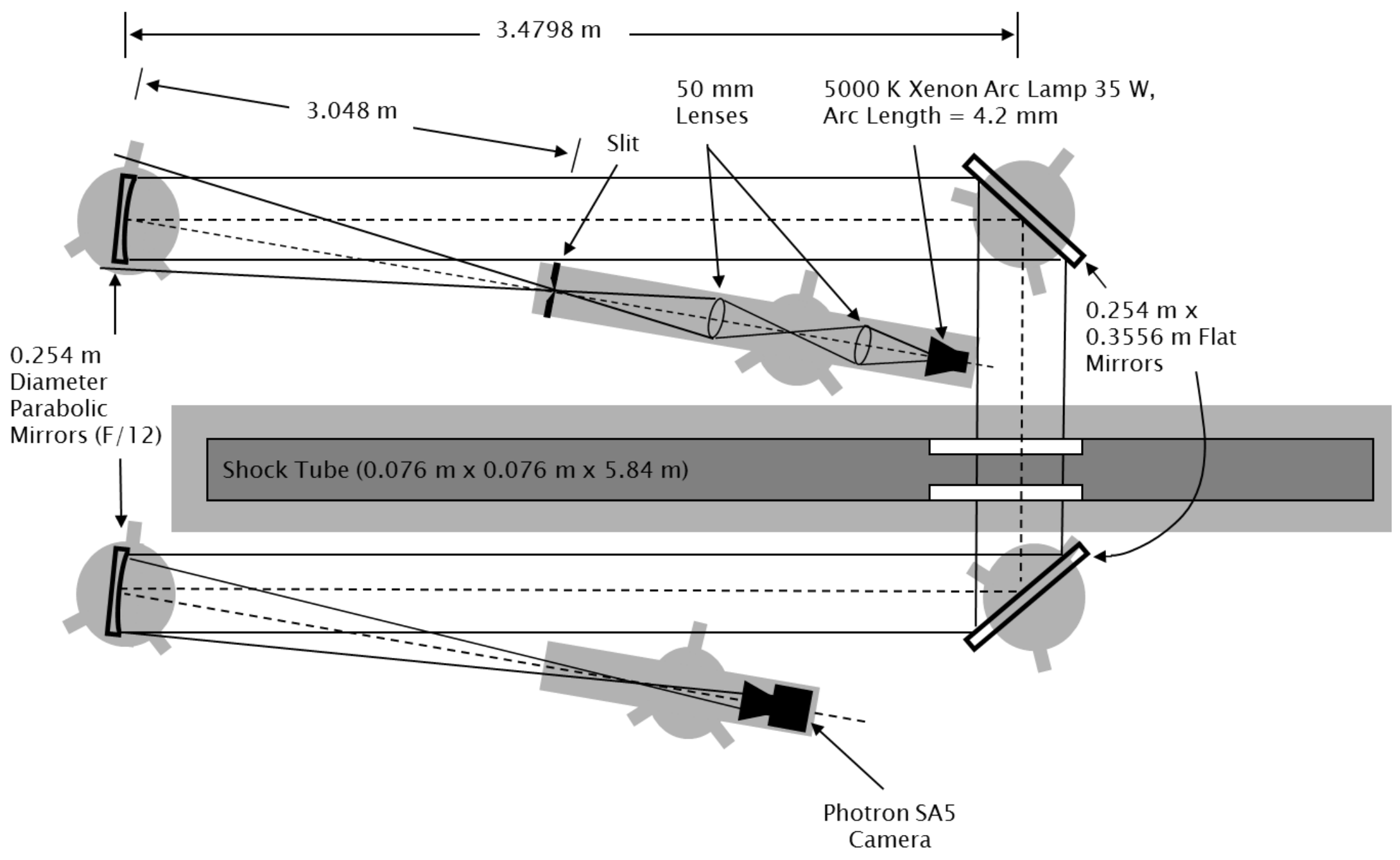

Figure 3.12: High-speed shadowgraph visualization setup [27] 
The light originates from a $35 \mathrm{~W}$ Xenon arc lamp, driven by a $12 \mathrm{~V}$ laboratory power supply. The light is passed through two focussing lenses and then through a small slit to block any stray light. It then falls on a parabolic mirror that is placed at a distance of $3.0 \mathrm{~m}$ from the slit. This distance is the focal length of the parabolic mirror, so all reflected light leaves as parallel rays. The parallel light is then reflected through the optical section of the shock tube using plane mirrors. Reflecting the light off another parabolic mirror has the effect of focussing the light at the mirror focal length.

A 70-300 mm Nikon telephoto lens was used for all shadowgraph videos. A wide-open aperture was required for all tests in order to capture as much light as possible. A framing rate of $37500 \mathrm{fps}$ at a spatial resolution of $768 \times 240$ was found to be adequate for the shadowgraph videos. This corresponds to a field of view of approximately $25.0 \mathrm{~cm}$ by $8.0 \mathrm{~cm}$.

\subsubsection{Direct Light}

With the acrylic tube inserted, it was not possible to pass the parallel light required for the shadowgraph video through the optical section. Because of this, a direct lighting setup was used for round cross-section side views. All end view videos, round and square cross-section, were directly side-lit through the channel optical glass. A $55 \mathrm{~mm}$ Nikon lens was used for all direct side view and head-on videos. Four $500 \mathrm{~W}$ halogen work lights were used as the lighting source with two placed on each side of the optical section for the $30^{\circ}$ angle and head-on views, as seen in Figure 3.13. All four lights were behind the shock tube for the side-view videos with a diffuser in front to even the light. This produced a back-lit video of the particle cloud. 


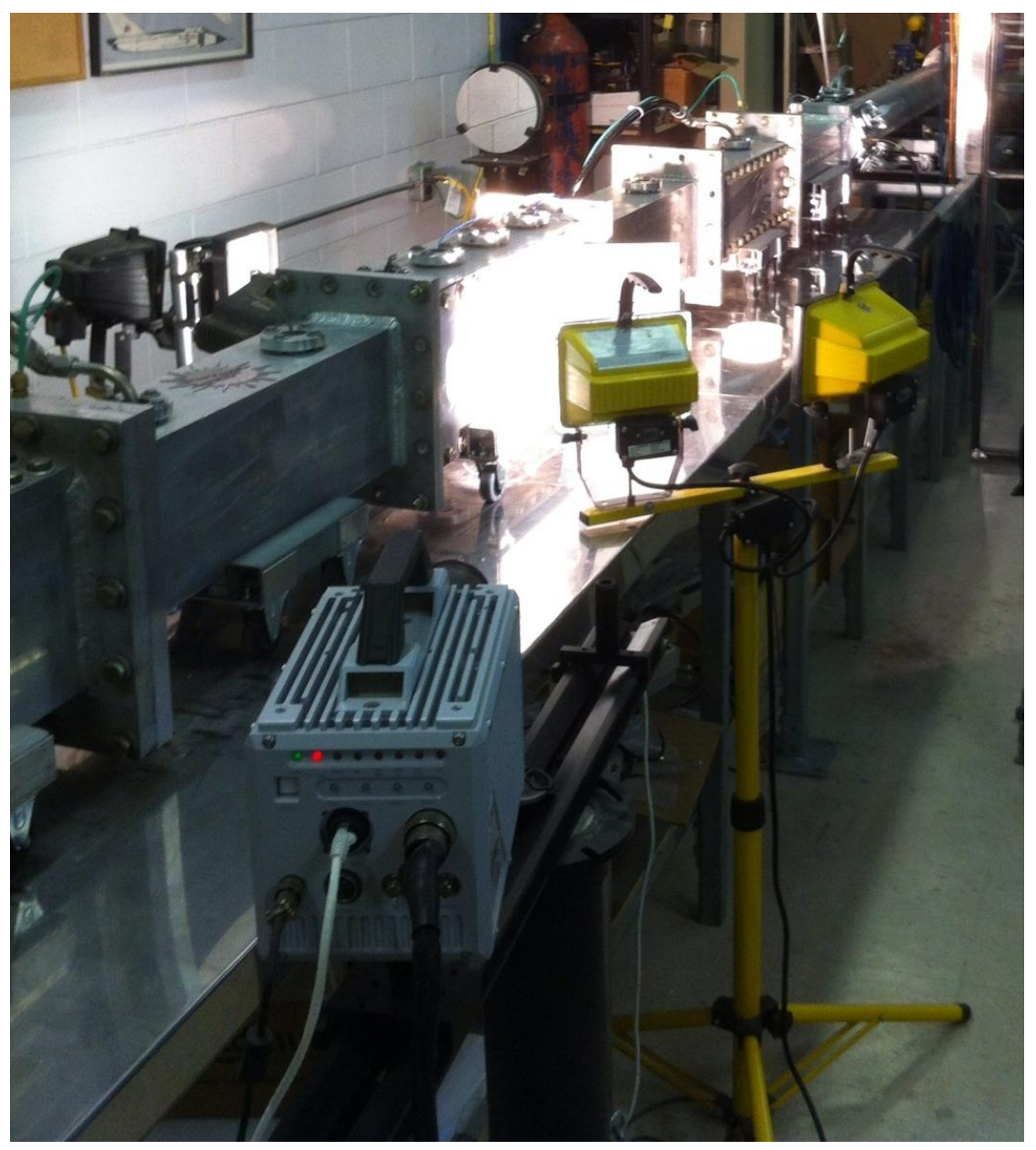

Figure 3.13: Camera and lighting setup for $30^{\circ}$ angle video

As in the shadowgraph videos, a wide-open aperture was used for the directly lit videos. Unfortunately, the larger the aperture, the shorter the resulting depth of field. This was not a problem with the side-view videos, as the angle being filmed allowed the particle cloud to stay a constant distance from the camera. On $30^{\circ}$ angle and head-on videos, the cloud is moving towards the camera. Because of this, the particle cloud was only in focus for approximately $10 \mathrm{~cm}$ in the stream-wise direction. This was overcome by filming all head-on tests at three different initial focal points: on the particle wafer, $5 \mathrm{~cm}$ downstream, and $10 \mathrm{~cm}$ downstream. A framing rate of $37500 \mathrm{fps}$ at a spatial resolution of $768 \times 240$ was found to be adequate for the side view 
tests, while a frame rate of $31000 \mathrm{fps}$ and a resolution of $448 \times 448$ were used for head-on views. This corresponds to a field of view of approximately $25.0 \mathrm{~cm}$ by $8.0 \mathrm{~cm}$ for side-view videos and $8.0 \mathrm{~cm}$ by $8.0 \mathrm{~cm}$ for head-on videos.

\subsection{Laser Density Measurement}

In an effort to determine the density of the particle cloud in time, a measurement system was built consisting of a laser and a photodiode to detect laser light. The laser light shone through the optical section normal to the window. The attenuation of the laser light is correlated with the cloud density. A $3 \mathrm{~mW}$ (Edmund Optics part number NT-39-569) diode laser with a wavelength of $670 \mathrm{~nm}$ and an oval beam of $2 \mathrm{~mm}$ wide by $5 \mathrm{~mm}$ high was used. The laser was placed in a position that was coincident with the location of either P6 or P7 (see Table 3.1), depending on the camera field of view that was being recorded. If high-speed video was taken in the first half of the optical section, the laser position was at P7. For video taken in the second half, the laser was at P6. Figure 3.14 shows the laser setup. 


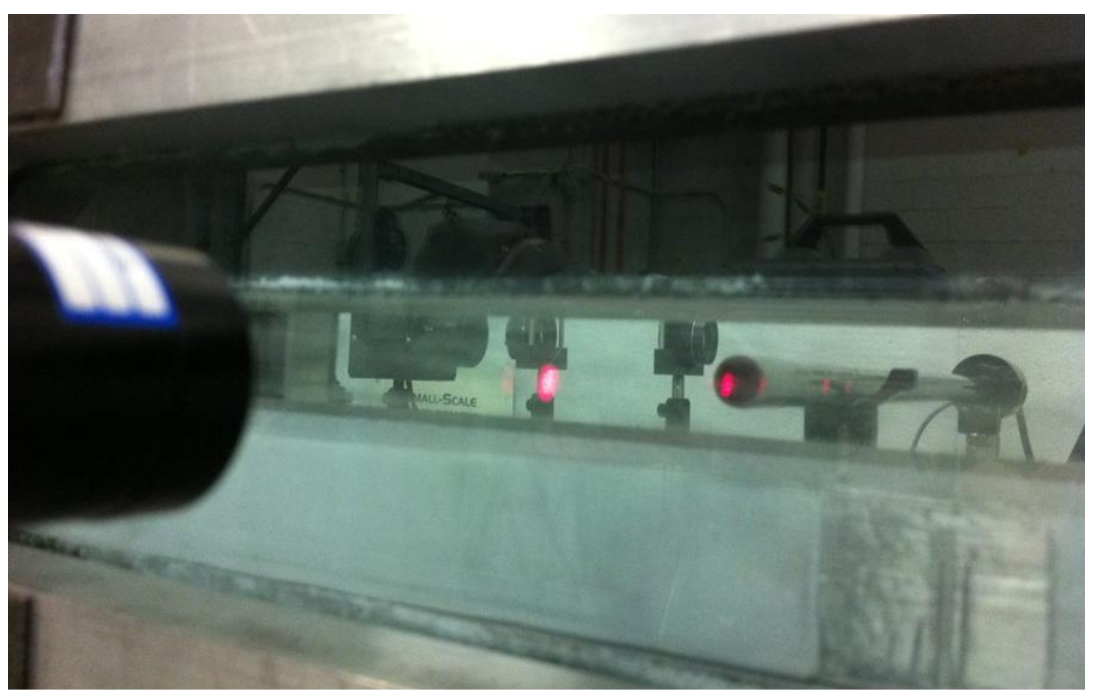

(a)

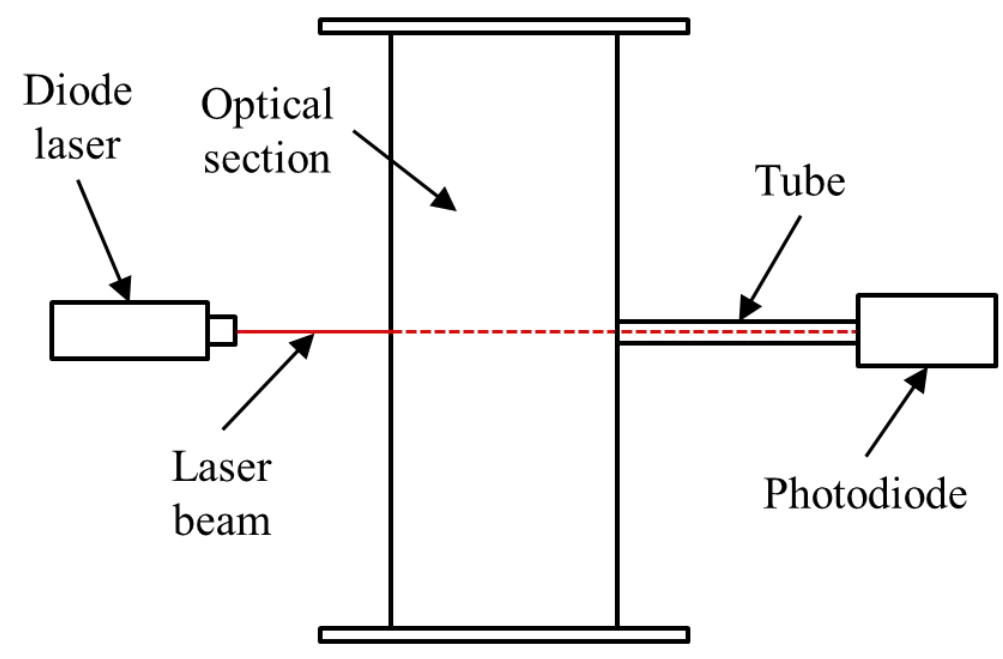

(b)

Figure 3.14: Laser-based cloud density measurement setup showing a) photo of laser through optical section and b) overhead view schematic

The beam entered the channel perpendicularly, measuring the cloud density over time at a fixed location. On the other side of the optical section a silicon photodiode (Edmund Optics part number NT57-625) with a detector diameter of $5 \mathrm{~mm}$ and a spectral response of $300 \mathrm{~nm}$ to 1000 
$\mathrm{nm}$ was placed to detect the laser signal. To ensure no stray light hit the photodiode, a $1.27 \mathrm{~cm}$ diameter tube was placed between the optical section glass and the detector. This caused the photodiode to only register light coming from the laser. All components were fixed to an optical rail to ensure proper alignment. The photodiode output was connected to the data acquisition system to record the signal over the duration of the dispersal event.

Calibration of the system was performed in a small acrylic $7.6 \mathrm{~cm}$ long, $4.45 \mathrm{~cm}$ diameter cylindrical vessel. A known amount of powder was placed on the bottom of the vessel and a high velocity jet of air is used to disperse the powder evenly throughout the vessel. The light attenuation through the particle cloud was recorded in terms of the drop in photodiode voltage output versus the average cloud density (total mass/vessel volume). The calibration was carried out in the range of cloud density of $13.5 \mathrm{~kg} / \mathrm{m}^{3}$ to $60.0 \mathrm{~kg} / \mathrm{m}^{3}$. The calibration provided the following linear relationship between the photodiode voltage drop, $\Delta V$, in $\mathrm{mV}$ and the cloud density, $\rho_{c}$, in $\mathrm{kg} / \mathrm{m}^{3}$

$$
\Delta V=-0.0075 \rho_{c}
$$

\subsection{Image Processing}

Videos were processed using the computer program ImageJ to create binary black-and-white frames using a color threshold to define the cloud area. The binary images where then processed using a code developed in MATLAB that allowed for the tracking of the particle cloud position. The cloud front was defined as the average of the leading and trailing edges of the front. The code outputs a text file that defines the cloud position (in pixels) in each video frame. The MATLAB code is given in Appendix B.

The camera frame rate and resolution were known and the pixel position information was given by MATLAB. Therefore, a time step and the number of pixels moved within that time step 
was found, giving a velocity. The distance downstream of the diaphragm and velocity of a feature are given by:

$$
\begin{gathered}
x_{C}=x_{w}+\frac{\varepsilon}{\alpha} \\
V_{C}=\Delta x_{C} f
\end{gathered}
$$

Where:

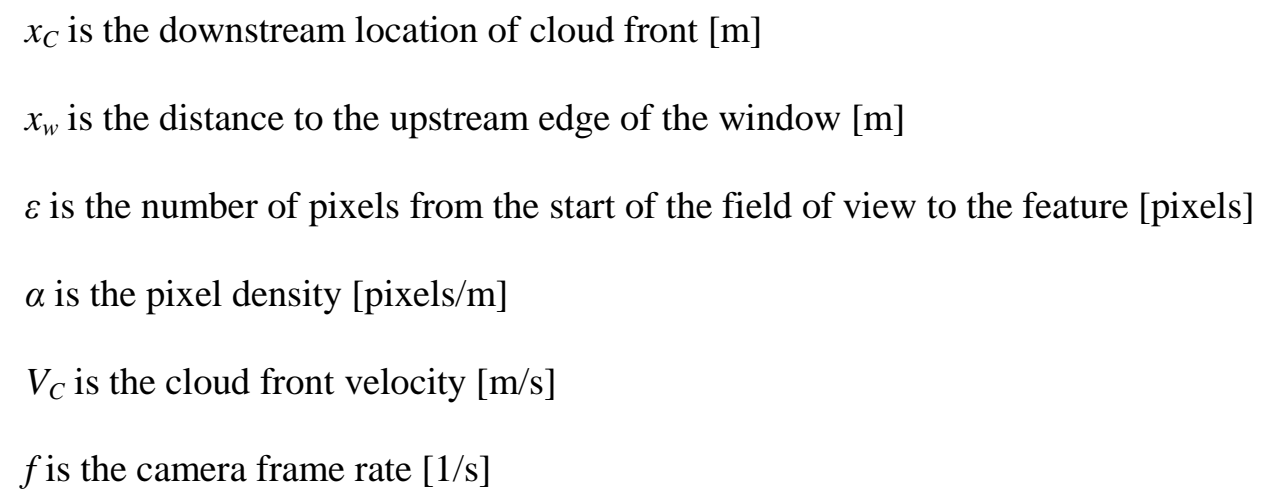

This technique was the method used to determine cloud acceleration and was used in conjunction with pressure sensor time-of-arrival data to determine shock wave velocities.

\subsection{Operating Procedure}

To initiate a test, the wafer was first created and loaded into the shock tube driven section. The diaphragms were then loaded into place on both sides of the diaphragm holder and the shock tube was clamped shut. All valves on the control panel were initially opened, except for valves 1 and 2. The vacuum pump was turned on and valves 4 and 6 were closed to begin the evacuation of the shock tube driver section and the diaphragm holder. Once a vacuum in these sections was achieved, valve 5 was closed and the vacuum pump was turned off. Valve 2 was then opened, 
letting helium into the driver and the diaphragm holder to the intermediate pressure of 7.55 bar. Valves 8 and 9 were then closed, closing off the diaphragm holder, and the driver was then filled to its final pressure of 11.34 bar. Closing valve 7 shut off the driver from the system. Next, the vacuum pump was turned back on and valves 5 and 8 were opened, evacuating the $500 \mathrm{~mL}$ vacuum volume. Once evacuated, all valves were closed and the system was ready to fire. To fire the shock tube valve 9 was opened, causing the helium in the diaphragm holder to be sucked into the vacuum volume. The first diaphragm immediately busts, as it's unable to withstand the pressure differential between the 11.34 bar driver and the now near-vacuum of the diaphragm holder. The second diaphragm bursts as it is also unable to withstand the 10.33 bar pressure differential across it. This results in a shock wave propagating through the atmospheric air in the driven section of the shock tube.

\subsection{Shock Tube Cleaning}

After a particle wafer test, cleaning of the shock tube was an integral part of the experimental process. After each test, the inside of the shock tube became covered in a layer of aluminum oxide powder, with most of it accumulating in the end of the driven section, between the optical section and the end plate. A novel cleaning technique was developed to easily and safely remove the majority of powder from the tube. An air distributer block was designed that, when connected to a compressed air line, blew powder off all four walls of the shock tube. A diagram is shown in Figure 3.15 . 


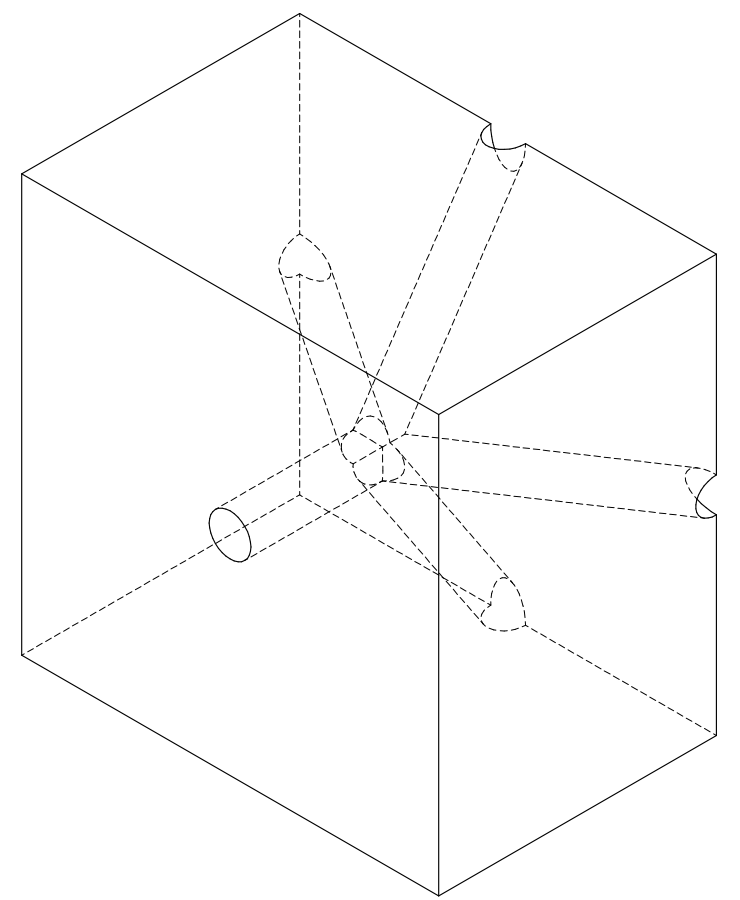

Figure 3.15: Air distributer used for cleaning powder from the inside of the shock tube

An end plate with a hole cut in the middle of an adapter allowed a shop vacuum cleaner to be attached to the end of the shock tube. With a high efficiency filter to trap the very small particles, the vacuum cleaner was turned on and began to collect powder from inside the tube. To aid this, the air distributor was inserted in the shock tube from the other end with compressed air blowing through it. The vacuum caused the distributer to be pulled down the length of the tube and along the way it blew particle off the wall so they could be collected by the vacuum cleaner. The motivation to using this cleaning method was that it was gentle on the windows. Since the aluminum oxide powder was so abrasive, any cleaning method that involved rubbing the powder off the windows would have caused damage to them, resulting in poor quality for the high-speed videos. Once the distributor had been run through the tube, a soft cloth was pushed through the tube to capture any remaining particles. Once clean, the shock tube was ready to run another particle dispersion test. 


\section{Chapter 4}

\section{Results and Discussion}

\subsection{Delrin $®$ Block}

Baseline tests were performed with a loose-fitting solid Delrin ${ }^{\circledR}$ block placed in the same location as the particle wafer (see Figure 3.6). For this configuration the block accelerates based solely on the pressure distribution produced following the shock interaction. The block was of the same mass as the particle wafer and was inserted inside the channel. The data from one such experiment with a helium driver pressure of 11 bar absolute is provided in Figure 4.1. The pressure traces recorded at P3 through P8 (see Table 3.1 for transducer locations) are shown in Figure 4.1. Figure 4.1 also represents a classic $\mathrm{x}$-t diagram that conveniently displays axial front trajectories, the slope of which represents the front velocity. For example, the high-speed video captured trajectories of the block and the shock wave that forms downstream of the block are shown in Figure 4.1 as boxes.

(B) Delrin is a registered trademark of DuPont for its acetal resins 


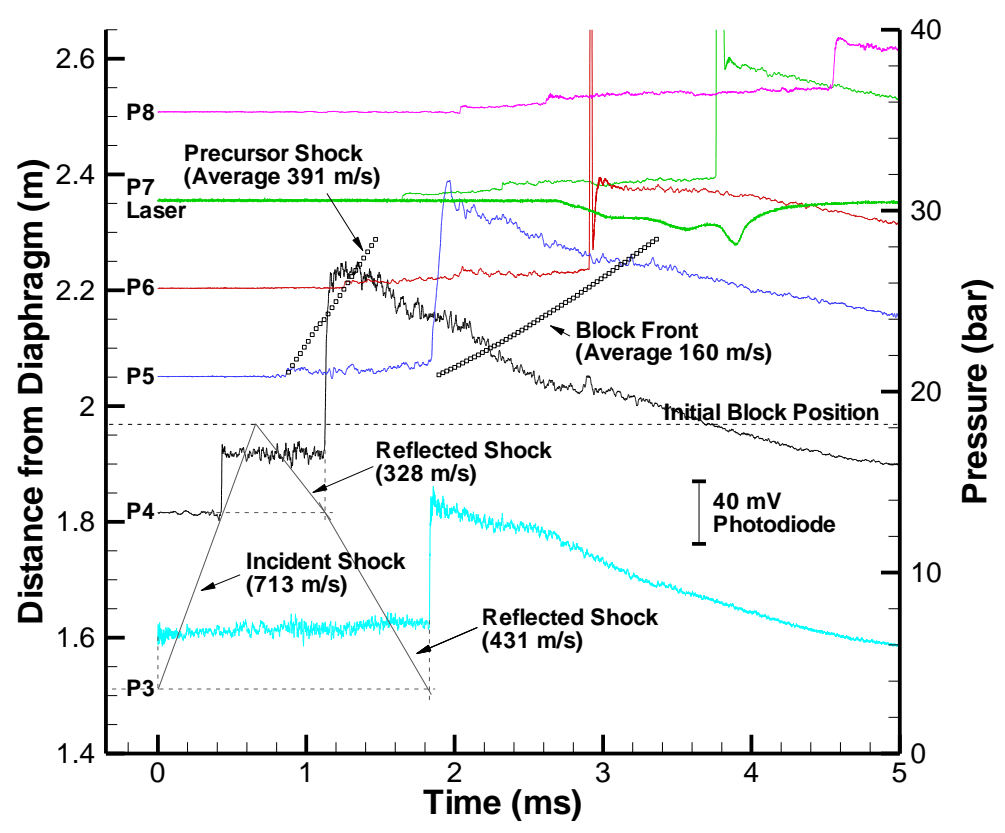

(a)

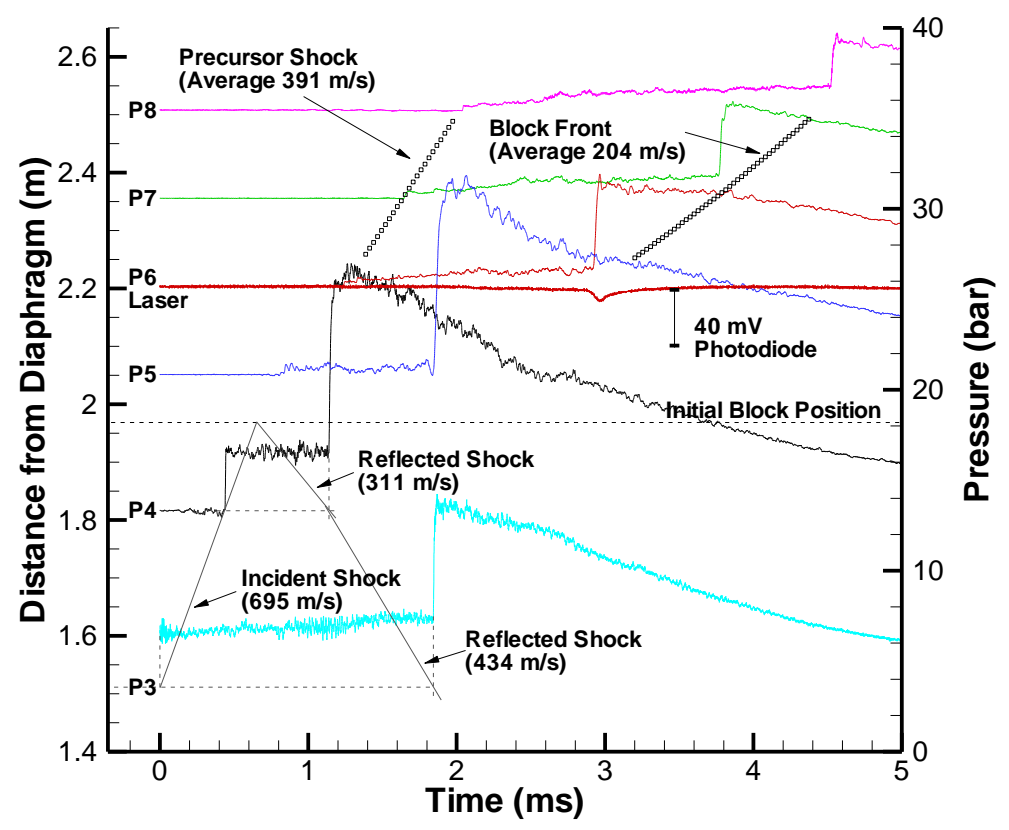

(b)

Figure 4.1: Diagram showing pressure traces as well as shock and block trajectories obtained from video images for an 11 bar abs. helium driver with the camera field-of-view (see Figure 3.6) covering (a) the first half of the optical section and (b) the second half of the optical section. 
The incident shock wave reflects off the upstream side of the block producing a reflected shock wave that propagates back towards the driver section. The reflected shock wave corresponds to the second pressure rise in the pressure signals recorded at P3 and P4 in Figure 4.1. A straight dotted line is shown connecting points on P3 and P4 (corresponding to shock time-of-arrival) showing the average trajectory of the incident and reflected shock wave. The $695 \mathrm{~m} / \mathrm{s}$ (Mach 2) incident shock wave produces a $311 \mathrm{~m} / \mathrm{s}$ and 14.5 bar reflected shock wave immediately after reflection that subsequently accelerates as it passes through the contact surface into the lower density helium driver gas. This compares to the theoretical fixed-surface normal reflection values of $348 \mathrm{~m} / \mathrm{s}$ and 15.2 bar. The deviation could be attributed to the initial block acceleration (not captured in the video) and high pressure air leakage past the block due to a loose fit. The interaction of the shock wave with the block results in acceleration of the block to a relatively constant velocity of $204 \mathrm{~m} / \mathrm{s}$, as shown in Figure 4.2. Unfortunately, the inside of the glass is fairly dirty looking from powder abrasion of previous tests. As a result, the shadowgraph images are not the best quality, but the main features as discussed below can be seen. 

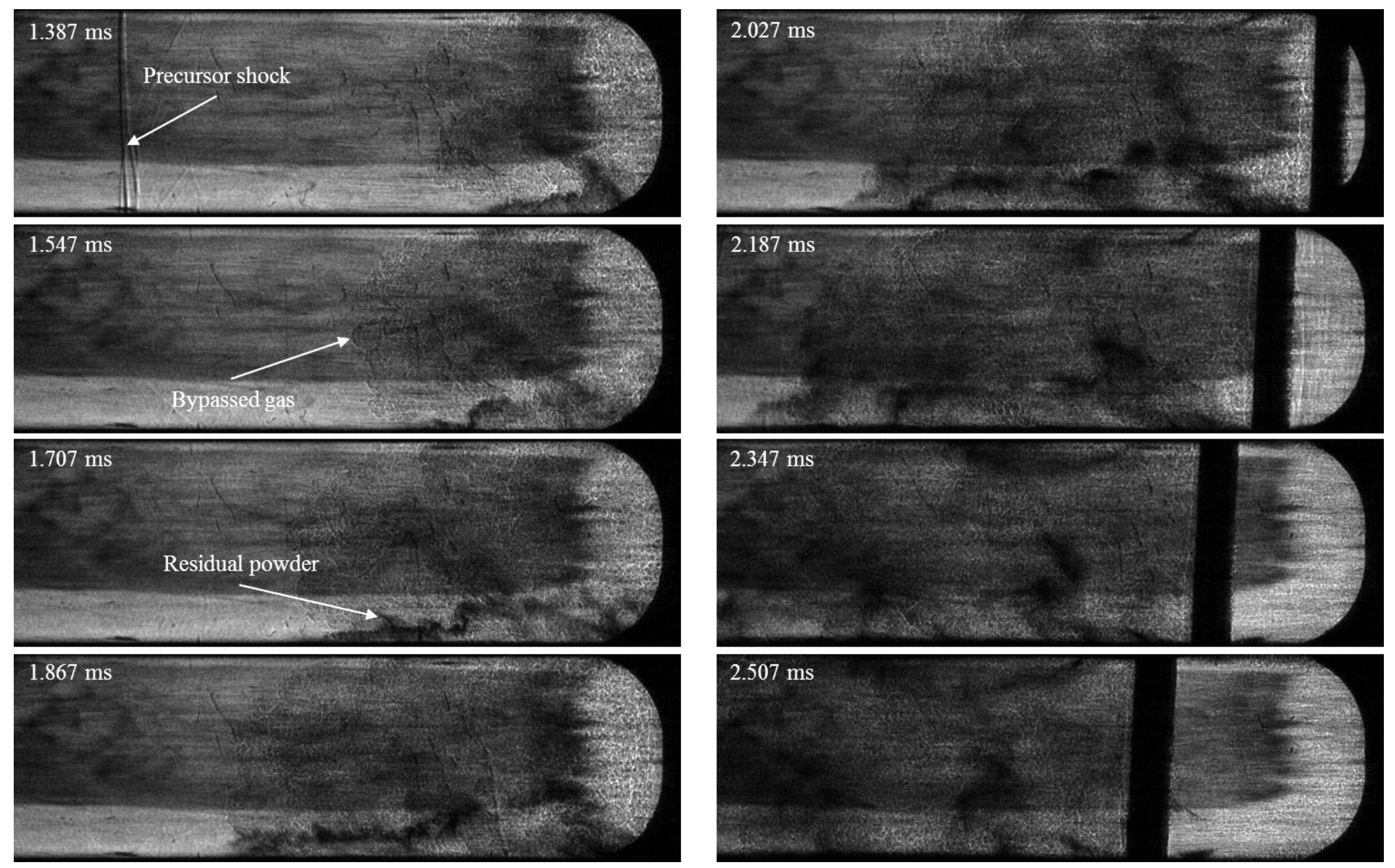

Figure 4.2: Images from 11 bar abs. helium driver showing acceleration of Delrin ${ }^{\circledR}$ block. Dark streak on gas due to abrasion from previous tests. Flow direction is from right to left 
The piston-like motion of the block produces a weak precursor shock, formed by the coalescence of multiple weak compression waves ahead of the block that propagates at a velocity of $391 \mathrm{~m} / \mathrm{s}$ as shown in Figure 4.1. The small amount of gas that initially bypasses the block around the edges also produces compression waves that contribute to the generation of the precursor shock wave. The acceleration of the block and bypassing of some of the reflected shock gas produces a shallow pressure gradient between the block and the precursor shock wave. The high pressure air upstream of the block moves with the block and is manifested as a travelling shock-like pressure pulse observed in the P5 to P8 signals in Figure 4.1. The pressure behind the block decays as the block propagates down the channel and the high pressure gas region expands as the distance between the block and the reflected shock increases.

A schematic showing the interaction of the shock wave and the block is shown in Figure 4.3. A reflected shock wave immediately forms, and a series of weak compression waves form ahead of the accelerating block that eventually forms a shock wave. 


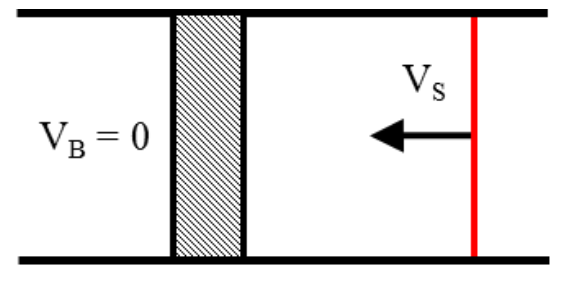

$\begin{array}{cc}\text { Delrin }{ }^{\circledR} & \text { Incident shock } \\ \text { block } & \text { wave }\end{array}$

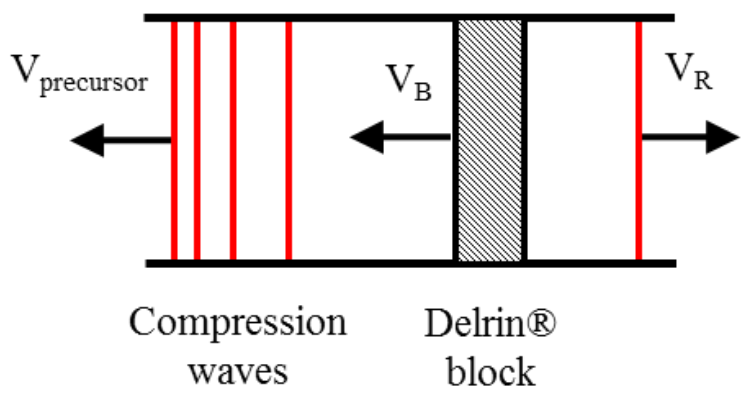

\section{Before shock-block interaction}

\section{After shock-block} interaction

Figure 4.3: Schematic showing shock wave-block impact event

\subsection{Particle Wafer - Square Channel Cross-section}

Results with the particle wafer obtained from two tests performed with a 6.5 bar absolute driver pressure and atmospheric pressure in the driven section is shown in Figure 4.4. The camera fieldof-view for the test results in Figure 4.4a consists of the first half of the window, see Figure 3.6.

For the results shown in Figure 4.4a, the incident shock wave velocity measured from the shock time-of-arrival between P3 and P4 is $581 \mathrm{~m} / \mathrm{s}$. The incident shock wave reflects off the particle wafer producing a reflected shock wave that propagates upstream at an average velocity of 316 $\mathrm{m} / \mathrm{s}$. The pressure behind the reflected shock wave measured at P4 remains relatively constant at about 8.5 bar over a period of roughly $0.4 \mathrm{~ms}$, after which time the pressure starts to drop off. Based on the incident shock wave velocity, the theoretical reflected shock velocity and pressure that would be produced upon reflection from a solid immobile surface are $333 \mathrm{~m} / \mathrm{s}$ and 8.4 bar, 
respectively. The close correspondence between the measured and theoretical reflected shock parameters, similar in magnitude with the block test shown in Figure 4.1, indicates that the back of the wafer remains largely intact after the reflection and there is very little initial forward movement of the wafer over this time. When the wafer starts to move forward, expansion waves propagate upstream towards the reflected shock wave dropping the pressure along the way. By the time the reflected shock wave reaches P3 the head of the expansion has caught the shock wave and the pressure behind the shock wave drops immediately. Similar to that observed with the block, the initial slow forward motion of the wafer produces compression waves that move ahead of the wafer. This causes the pressure at transducer P5 to rise slowly. A pressure front forms at P6 that steepens with distance as the compression waves merge. At P8, the compression waves have coalesced to form a shock wave characterized by a steep pressure rise at roughly $3 \mathrm{~ms}$ in Figure 4.4a. 


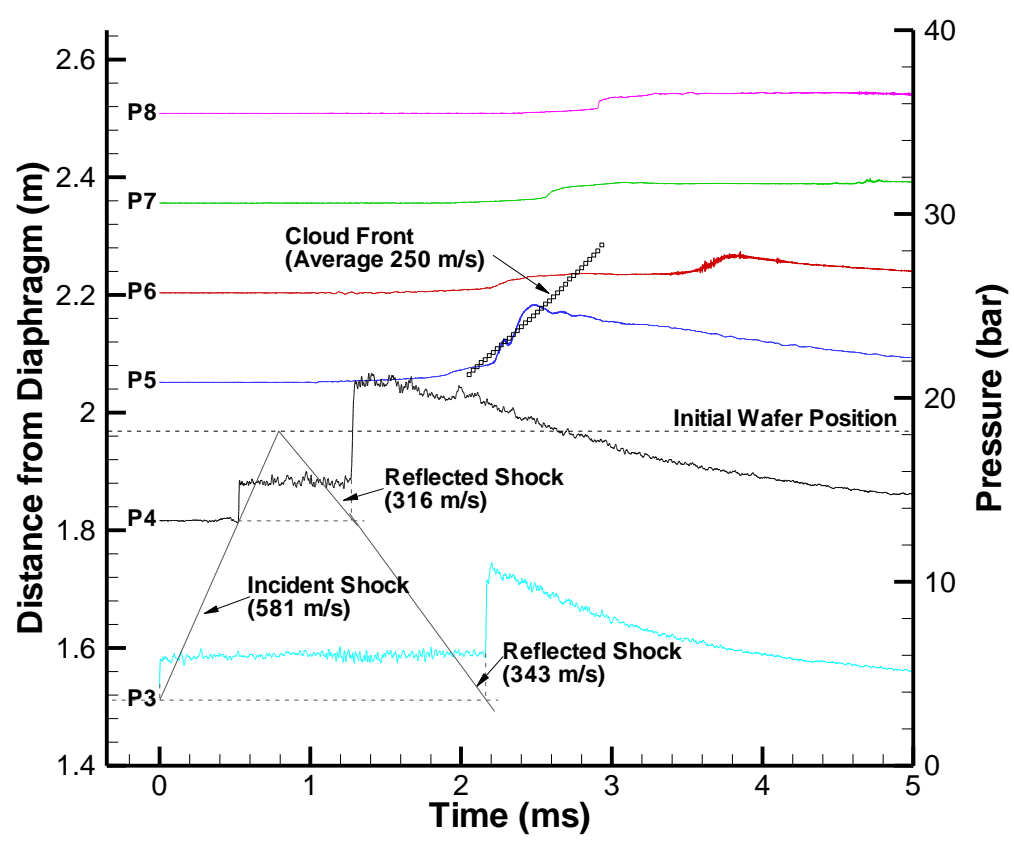

(a)

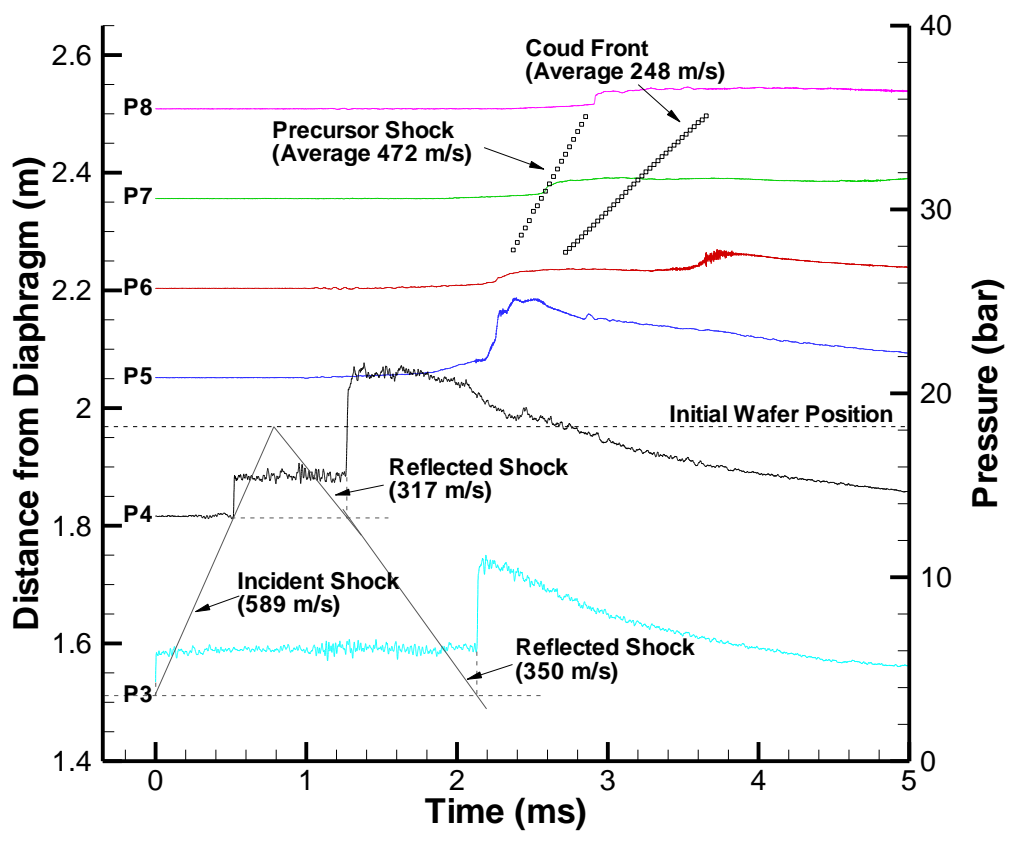

(b)

Figure 4.4: Diagram showing pressure traces for a 6.5 bar abs. helium driver. Shock and cloud front trajectories from videos with the camera field-of-view (see Figure 3.6) covering (a) the first half of the optical section and (b) the second half of the optical section 
As long as the back edge of the particle wafer remains intact, it acts like a piston driven by the high pressure produced by the reflection of the incident shock wave. The rear edge of the wafer passes P5 at $2.2 \mathrm{~ms}$ when a large pressure rise is recorded, see Figure 4.4a. The finite pressure rise-time recorded at P5, compared to the sharp pressure rise observed for the block in Figure 4.1, indicates that the back of the wafer is not completely intact and that there is some high pressure air bypassing the wafer. The pressure pulse propagates forward at a velocity slower than the precursor shock wave and the magnitude of the pressure pulse dramatically decreases with distance. Also shown in Figure 4.4a is the position of the front edge of the particle cloud taken from high-speed video images shown in Figure 4.5. The front edge of the cloud, defined as the average between the leading and trailing edges of the cloud front, moves at a velocity of $250 \mathrm{~m} / \mathrm{s}$. From Figure 4.4a it is clear that the front edge of the cloud passes the transducer P5 position before the large pressure pulse, which is associated with the passage of the back edge of the particle cloud. More importantly is the fact that the distance between the front edge and back edge (deduced from the pressure pulse) of the cloud increases with propagation distance. 

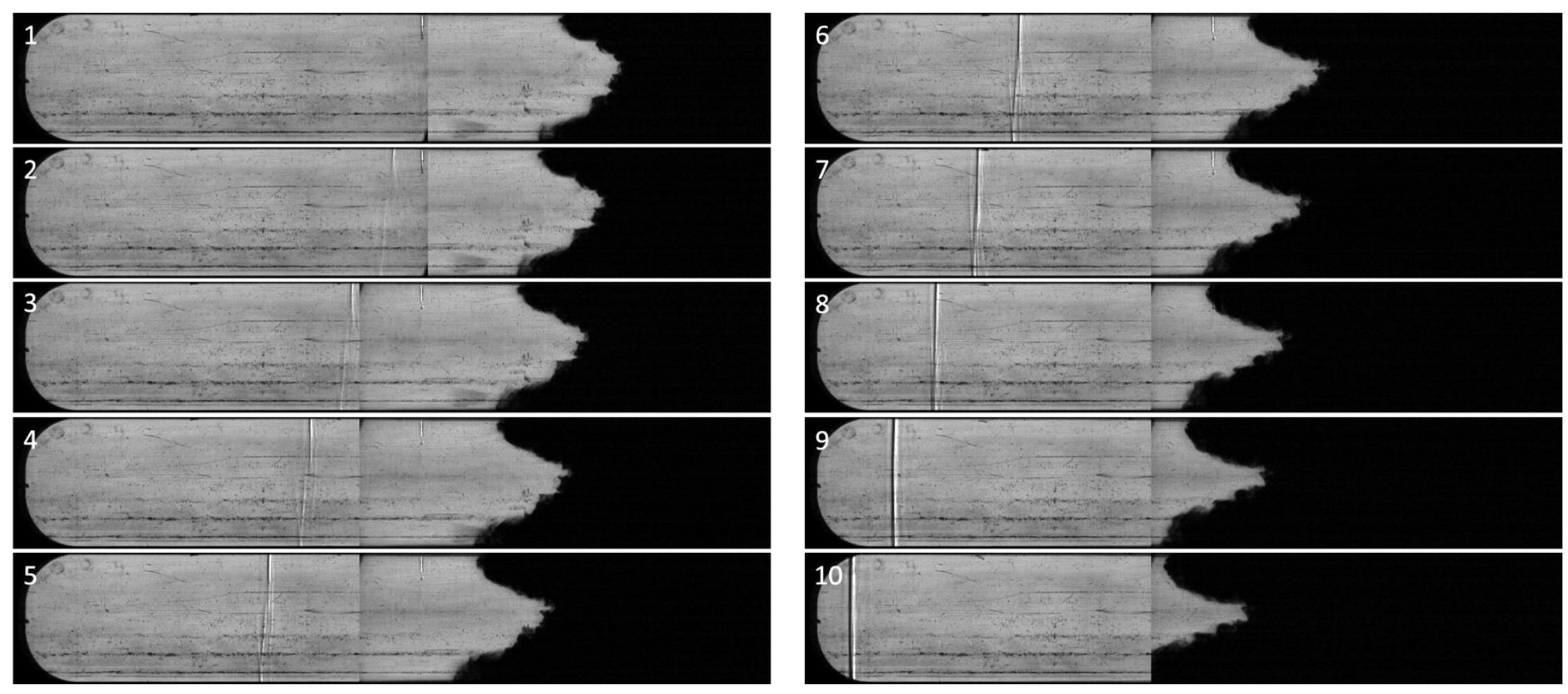

Figure 4.5: Compilation of two videos showing the non-uniform particle cloud leading-edge and the shock wave development ahead of the particle cloud. From tests with 6.5 bar abs. helium driver. Inter-frame time is $0.027 \mathrm{~ms}$. Flow direction is from right to left 
Figure 4.5 shows a compilation of high-speed video images taken from the two tests represented by Figure 4.4. The initial wafer location is outside the field of view, $8.5 \mathrm{~cm}$ beyond the right edge of the window frame. Time zero corresponds to the elapsed time after the shock wave reaches the P3 location (trigger for camera) shown in Figure 3.6. As the cloud front edge moves forward, a weak compression wave forms that eventually is reinforced to form a shock wave by the left edge of the field-of-view, corresponding to the transducer P8 location in Figure 3.6. The back edge of the cloud is not viewable because particles become trapped in the slow moving boundary layer flow next to the windows. Furthermore, particles acquire a static charge during their motion and adhere to the glass windows. The position of the front edge of the particle cloud and the forming shock wave obtained from the video images from a test where the camera field-of-view covers the second half of the window is plotted in Figure 4.4b. The velocity of the shock wave obtained from the video images is $472 \mathrm{~m} / \mathrm{s}$. The shock wave position obtained from the video agrees very well with the pressure rise recorded at P7 and P8. The video images indicate that the front edge of the cloud propagates at a velocity of $248 \mathrm{~m} / \mathrm{s}$. This measured cloud front edge velocity is higher than the theoretical post-shock gas velocity of $181 \mathrm{~m} / \mathrm{s}$ behind the $472 \mathrm{~m} / \mathrm{s}$ precursor shock wave. This is an indication that the particles are not simply "riding" on the post-shock flow.

Results obtained from tests performed with an 11 bar absolute driver pressure and atmospheric pressure in the driven section are shown in Figure 4.6. The test results in Figure 4.6a show an incident shock velocity of $698 \mathrm{~m} / \mathrm{s}$. The reflected shock pressure at $\mathrm{P} 4$ is 13 bar, which is lower than the theoretical value of 15.2 bar. The motion of the wafer produces a shock wave velocity of $507 \mathrm{~m} / \mathrm{s}$ measured between $\mathrm{P} 7$ and P8 (not shown in Figure 4.6a). Between transducers P6 to P8, the precursor shock wave is followed by a well-defined pressure rise associated with the back edge of the particle cloud. As observed in the 6.5 bar driver tests shown in Figure 4.4, the pressure rise-time increases as the wafer develops into a dense particle cloud. Based on the video, the velocity of the front of the cloud is $319 \mathrm{~m} / \mathrm{s}$, which again is significantly 
faster than the theoretical particle velocity of $233 \mathrm{~m} / \mathrm{s}$ behind the $515 \mathrm{~m} / \mathrm{s}$ precursor shock wave. The arrival of the back edge of the cloud can be inferred from the arrival of the second pressure pulse at P6, P7 and P8. This is in contrast to the fast rise-time, shock-like pressure pulse associated with the moving solid block in Figure 4.1. The pressure rise is spread out over the cloud thickness and the exact location of the cloud back edge must be after the pressure maximum due to expansion dispersion into rear flow. The data from a test performed under similar conditions where the camera field-of-view covers the second half of the window is given in Figure 4.6b. The arrival of the back edge of the cloud at P6 is again identified after the pressure maximum at $3 \mathrm{~ms}$. 


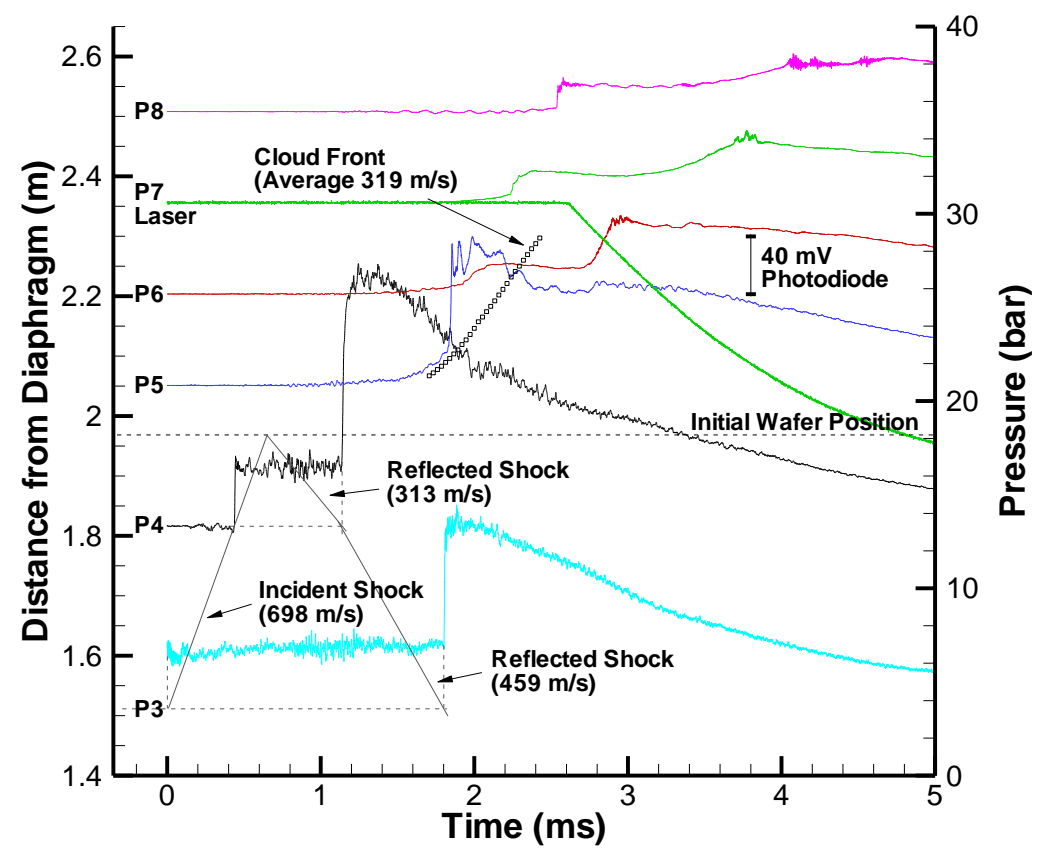

(a)

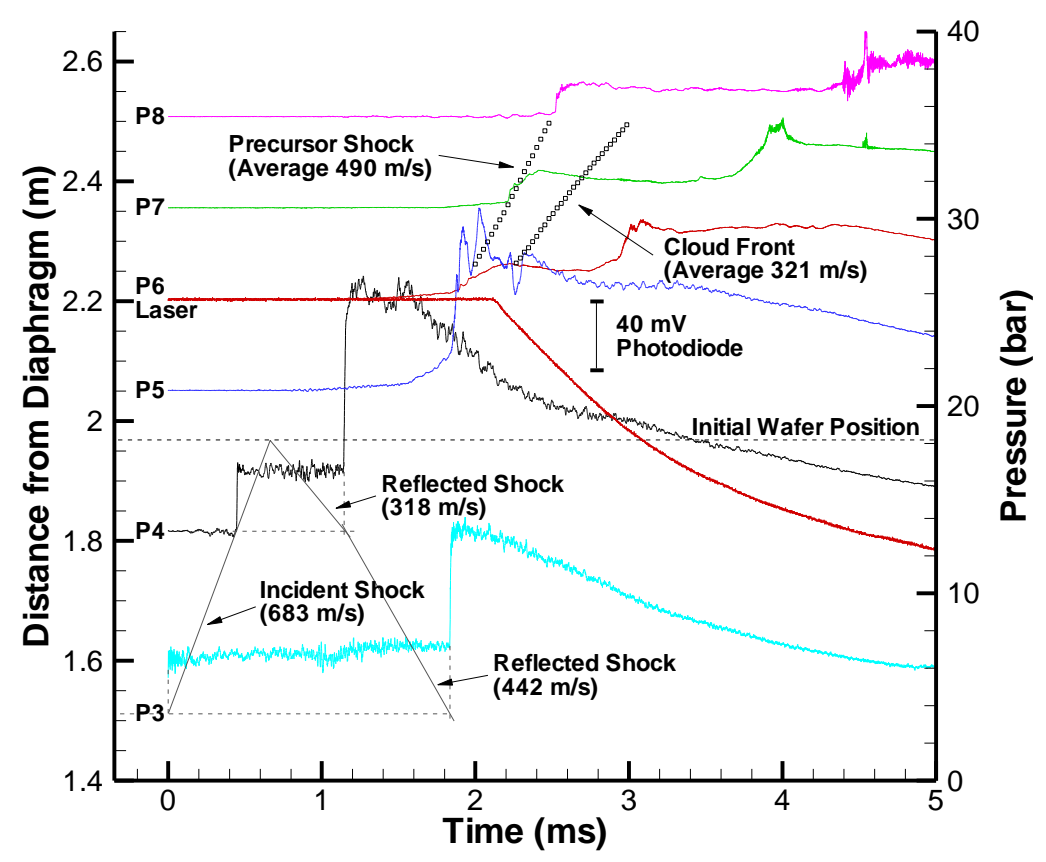

(b)

Figure 4.6: Diagram showing pressure traces for an 11 bar abs. helium driver. Shock and block trajectories with the camera field-of-view covering a) the first half of the optical section and $b$ ) the second half of the optical section 
Experiments were performed to visualize the reflected shock produced for the interaction of a 690 $\mathrm{m} / \mathrm{s}$ incident shock wave off both a Delrin ${ }^{\circledR}$ block and a particle wafer. To do this, the sample was placed at the flange located at the end of the optical section, just after the location of P8. Video images of the reflected shock as it propagates upstream are compared directly in Figure 4.7. The shock and flow structure in both cases are identical, indicating a similarity in the shockblock/wafer interaction. Both reflected shocks were found to be moving at $313 \mathrm{~m} / \mathrm{s}$ from time of arrival at P8 and P7. The reflected shock pressures were also found to be the same, 12.9 bar, at P7 and P8. The reflected shock wave interacts with the boundary layer which produces a complex "lambda-shock" structure near the wall [28]. The boundary layer associated with the incident shock (ahead of the reflected shock), as well as the "lambda-shock" configuration near the wall are highlighted in Figure 4.7b. The flow perturbations behind shock wave are most likely associated with the turbulent boundary layer separation associated with the shock-boundary layer interaction. 

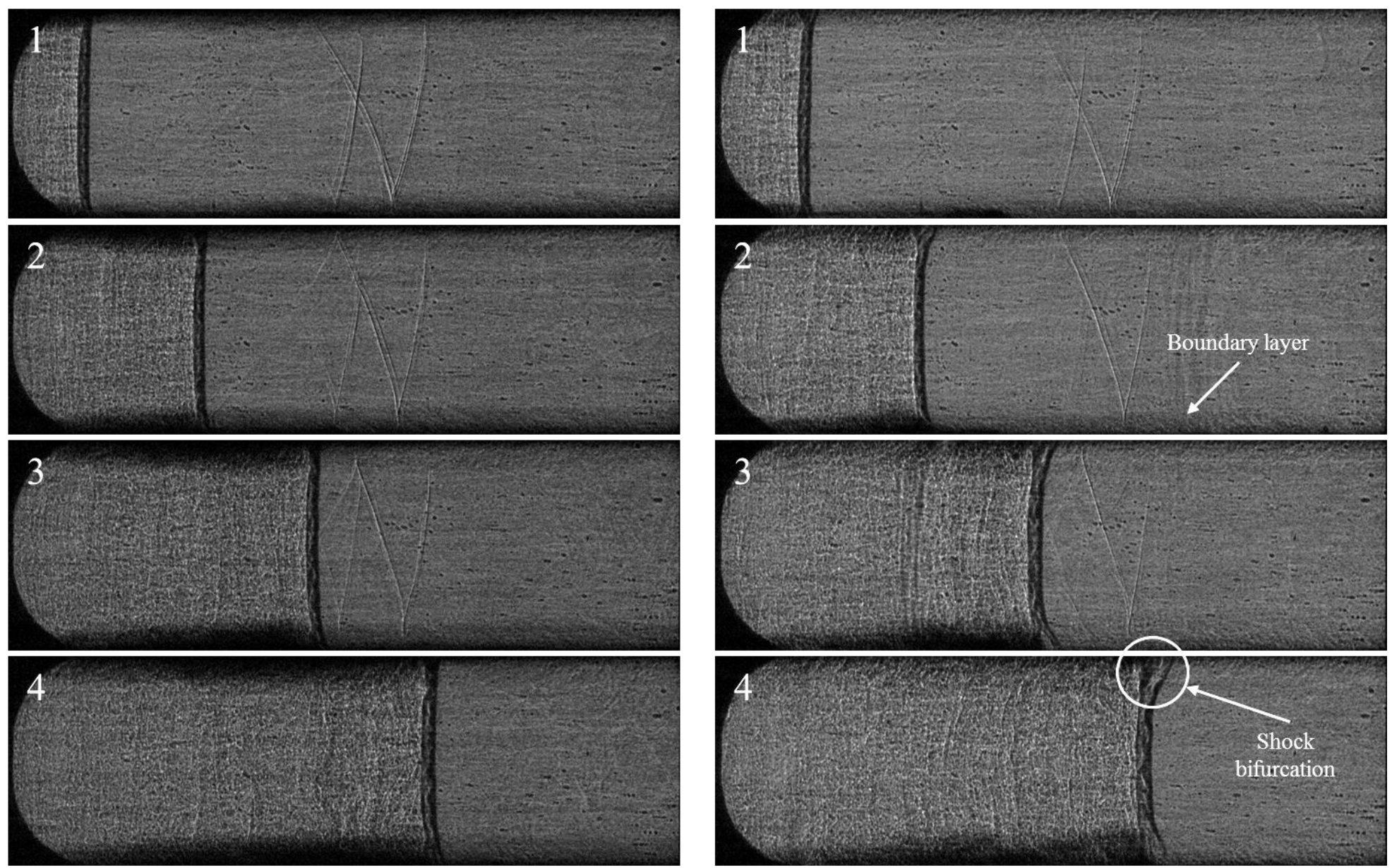

(a)

(b)

Figure 4.7: Reflected shock comparison between a) particle wafer and b) Delrin® block from a 11 bar abs. helium driver. Inter-frame time is $0.134 \mathrm{~ms}$ 


\subsection{Cloud Density Measurement}

Using the laser-based system described in Section 3.5, cloud densities were measured during the dispersion events. Shown in Figure 4.1 is the photodiode signal from a test with a Delrin ${ }^{\circledR}$ block, approximately $6.3 \mathrm{~mm}$ thick. In this test, the photodiode was located at the same position as P6 and the pressure transducer and photodiode signals both indicate the arrival of the plug at the same time. The photodiode signal is characterized by a drop in voltage as the laser light is attenuated. The photodiode output voltage drop is very small since the time-of-flight for the block across the $2 \mathrm{~mm}$ wide laser beam is on the order of the response-time of the photodiode.

The laser system was also used in particle wafer tests, as can be seen in Figure 4.6a. When the front edge of the cloud interrupts the laser there is a drop in the photodiode output voltage. The start of the drop in the photodiode voltage at $2.7 \mathrm{~ms}$ agrees well with extrapolation of the trajectory of the front edge of the cloud from the video. There is no recovery in the voltage due to interruption of the laser by particle adhesion to the windows. This is the same reason why the video does not show the back edge of the cloud.

The photodiode voltage does not drop sharply when the particle cloud passes by the laser beam. This could be attributed to a density gradient within the front of the particle cloud. To confirm this, tests were performed with a $5.47 \mathrm{~cm}$ thick block of high-density foam. Using the thicker block was beneficial because the time-of-flight for the plug across the laser beam was much longer than with the Delrin ${ }^{\circledR}$ block discussed above. Results from this test are given in Figure 4.8. 


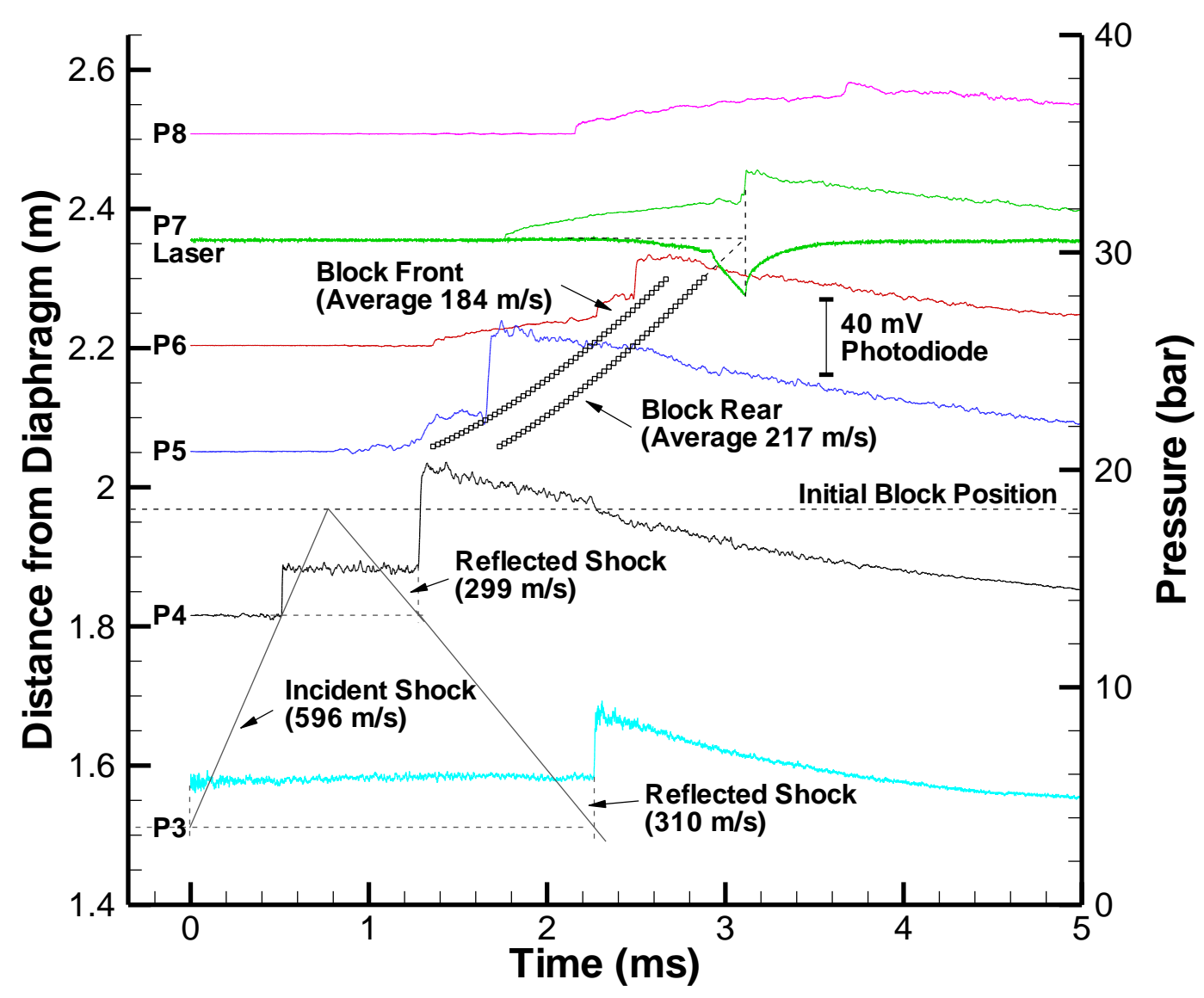

Figure 4.8: Diagram showing pressure traces for a 6.5 bar abs. helium driver with a $5.47 \mathrm{~cm}$ thick high-density foam block

Included in Figure 4.8 are the trajectories of the front and rear of the dense foam block taken from the video data. The front and rear of the block appear to get closer together due to compression of the foam. In the laser signal, a slight drop in voltage can be seen at approximately $2.5 \mathrm{~ms}$ due to residual aluminum oxide powder getting lofted in front of the moving block. Just as was seen in the Delrin ${ }^{\circledR}$ block test, there is only a very small drop in voltage as the block crosses the laser. With a block of this thickness, the passage of the block should produce a signal consisting of a very sharp drop in voltage to a constant value, associated with complete laser obstruction, followed by a sharp recovery in the voltage. This is not seen in the photodiode signal in Figure 
4.8. The peak voltage drop in the signal corresponded to the maximum response of the photodiode. The passage of the back of the block at P7 obtained from video matches well with the sudden increase in pressure and a sudden increase in the photodiode signal. Comparing the voltage drop observed with the thick foam block to that initially seen in the particle wafer tests reveals that the cloud density in the experiments is too high, effectively completely obstructing the laser beam. This signal saturation was not observed during laser calibration tests because the cloud densities were much lower than that achieved in the wafer tests. Another issue that reduced the effectiveness of the laser density measurements was the tendency for the particles to adhere to the windows where the laser was located. The channel width also could have had an effect on the accuracy of the laser density measurements as the stochastic distribution of the particles in the span-wise direction may have resulted in greater laser attenuation.

\subsection{Wafer Density Variation}

In order to see how the initial wafer density affected the particle dispersal, two additional wafer samples were created. To go along with the "standard" wafer with a particle volume fraction of 0.48, a "light" and a "heavy" particle wafer were also made using the die plate method with particle volume fractions of 0.38 and 0.58 , respectively. The light wafer was created using $55 \mathrm{~g}$ of aluminum oxide powder and the heavy wafer was made using $85 \mathrm{~g}$ of aluminum oxide powder. These particle volume fractions were chosen as they were the lower and upper limits that would produce a $7.6 \mathrm{~cm}$ square wafer within the $6.4 \mathrm{~mm}$ thick plate that would remain intact during shock tube preparation. Driver pressure for all tests was 11 bar. Experimental cloud front velocities are shown in Figure 4.9. 


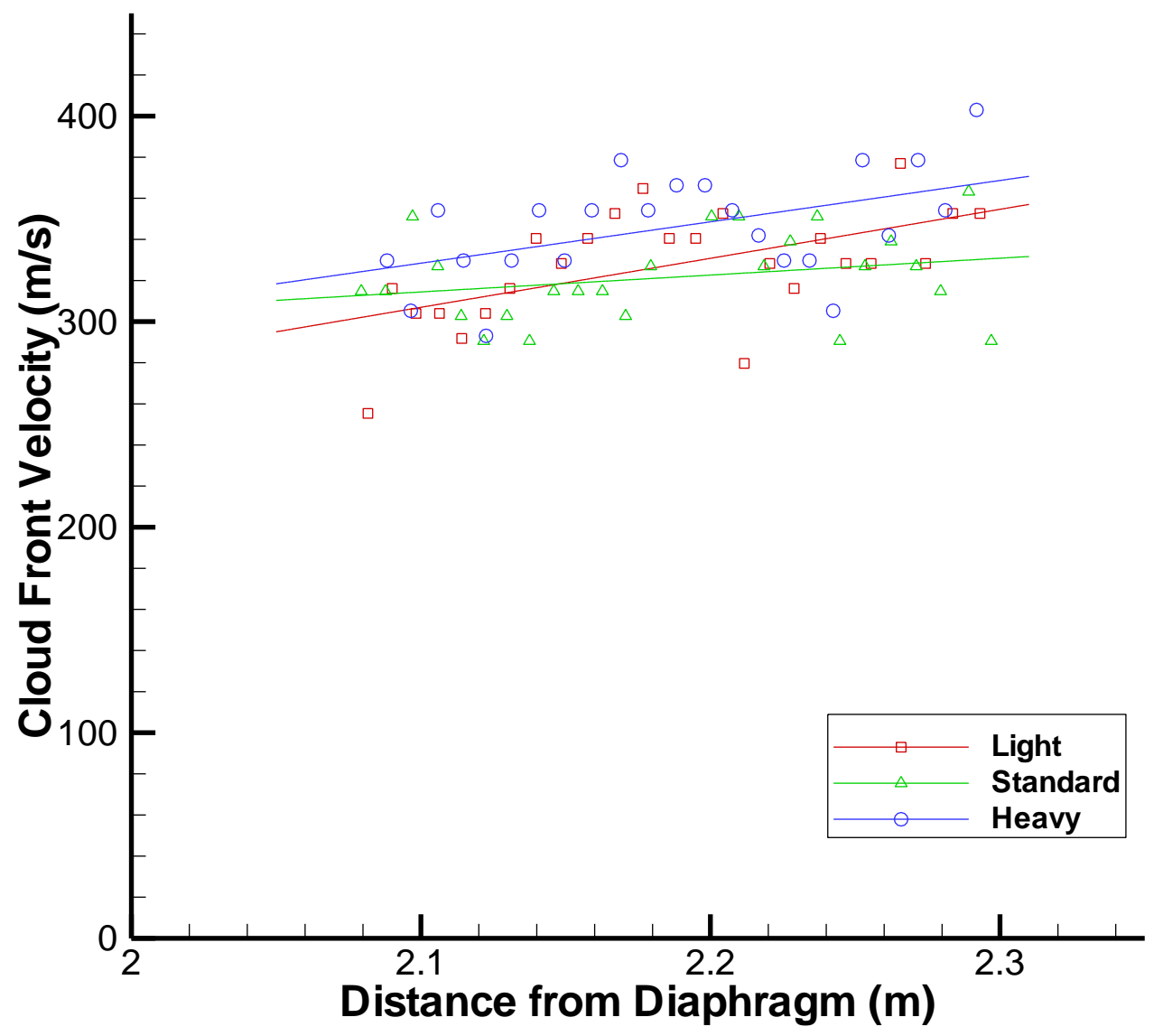

Figure 4.9: Cloud front velocity for varying particle volume fractions of initial wafer

With consideration of the substantial scatter, the data in Figure 4.9 shows cloud front velocities to be similar for all particle volume fractions. For the light particle wafer, the average cloud front velocity was found to be $344 \mathrm{~m} / \mathrm{s}$, while for the heavy wafer the velocity of the cloud front averaged $327 \mathrm{~m} / \mathrm{s}$. This is very similar to the measured velocity of the standard wafer of $320 \mathrm{~m} / \mathrm{s}$. This is surprising since if the wafer behaves as a solid block, one would expect the front velocity of the lower density wafer to be higher. However, as will be discussed below, the velocity of the front edge of the particle cloud is not the same as the wafer velocity.

The pressure traces at P4 in Figure 4.10 shows that the reflected shock pressure is higher for the higher density which is consistent with the expected lower wafer velocity. Also, when the 
incident shock reflects off the upstream face of the wafer, energy is lost to the compression of the wafer. With the dense wafer, there is less air space between particles for a compression to take place, so it acts more closely to the behaviour seen with a solid body. 


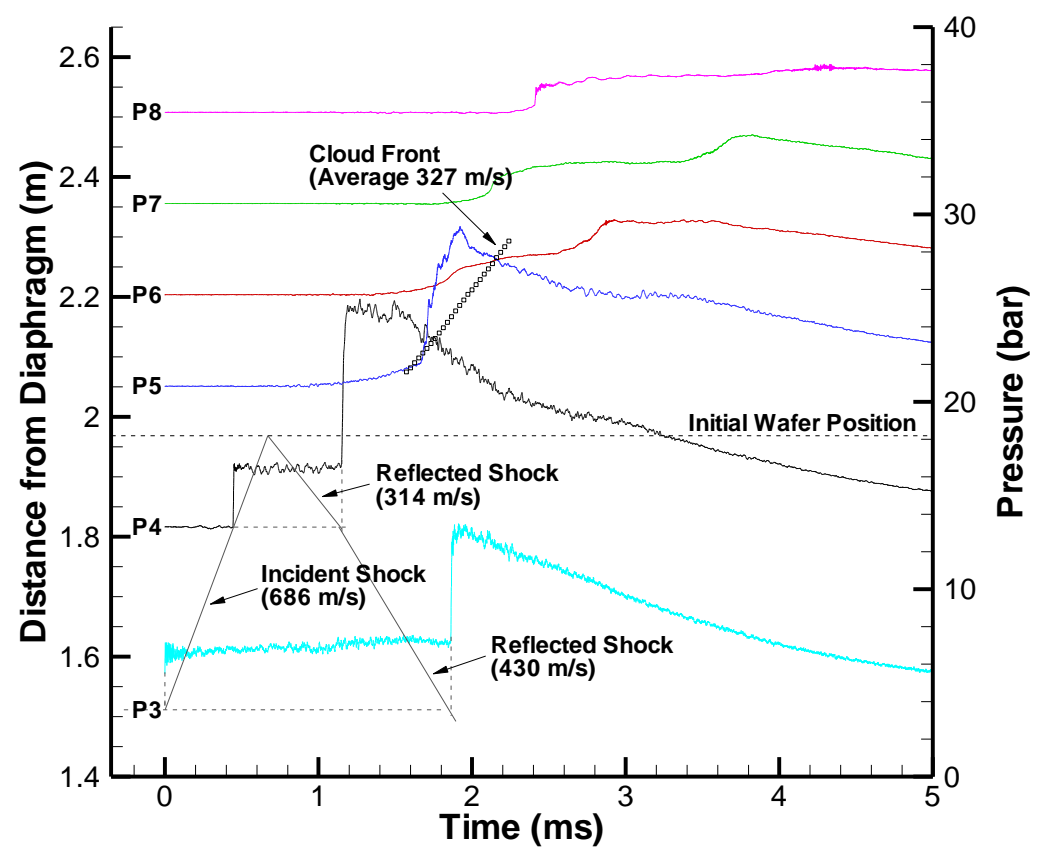

(a)

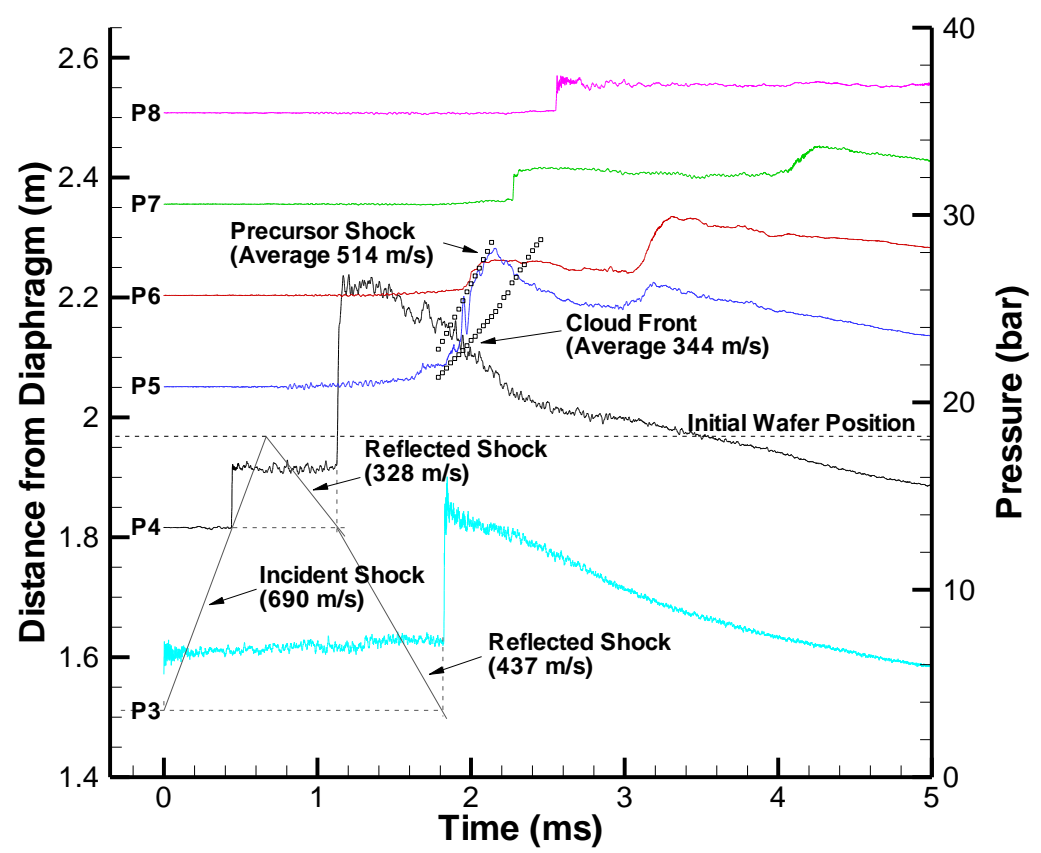

(b)

Figure 4.10: Pressure trace taken comparing pressure wave structure of a) $\varphi_{p}=0.38$ and b) $\varphi_{p}=\mathbf{0 . 5 8}$ particle wafers 
The reflected shock pressures measured at P4 were 11.4 bar and 12.9 bar for the light and heavy particle wafer respectively. This corresponds to a reflected shock wave velocities of $314 \mathrm{~m} / \mathrm{s}$ for the $\varphi_{p}=0.38$ wafer and $328 \mathrm{~m} / \mathrm{s}$ for the $\varphi_{p}=0.58$ particle wafer. From Figure 4.10, it is also clear that the compression waves coalesce further upstream in the case of the heavy wafer as shown by the steep pressure rise in P7 at $2.4 \mathrm{~ms}$ in Figure 4.10b. From this, it can be deduced that the $\varphi_{p}=$ 0.58 wafer produced a particle cloud that is more dense and allows less of the high pressure reflected gas to percolate through the cloud. This is also supported by the larger pressure pulse seen at $3.2 \mathrm{~ms}$ in Figure $4.10 \mathrm{~b}$ at P6. While the pressure pulse associated with the passage of the particle cloud at P5 is of the same magnitude in both parts of Figure 4.10, the pressure rise time increases quickly in Figure 4.10a, indicating the light particle wafer quickly becomes a permeable interface.

\subsection{Cloud Front Shape}

An interesting feature that can be seen in Figure 4.5 is the non-uniform, inverted "V" shape of the cloud leading-edge that is produced. The non-uniform leading edge develops very quickly, as it is present when the particle cloud enters the field-of-view. As the cloud moves downstream, the depth of the cloud leading-edge trough increases. This indicates that the particles near the channel wall are moving faster than particles at the center of the channel. The cloud shape is surprising because boundary layer theory would predict a parabolic shaped velocity profile with a velocity deficit at the walls that the particles would be expected to follow. A series of tests were performed to resolve questions concerning the cloud front-edge shape. The top and bottom surface of the channel are constructed of aluminum that have a different surface finish than the glass sides. To address this asymmetry, the top and bottom aluminum plates were replaced with glass plates in order to make all the channel inner-surfaces similar. With all channel surfaces made of glass, the "V" shaped profile persisted. 
The cloud front-edge observed in Figure 4.5 is in contrast to the planar cloud front-edge obtained by Wagner et al. [12]. Experiments were performed with a wafer that does not span the full width of the channel, a similar geometry to the particle curtain used in Wagner et al. [12]. The thinking was that the corners of the square cross-section wafer affected the cloud shape. A particle wafer was created with $12.7 \mathrm{~mm}$ cut away from the sides with a knife after the die was removed. Tests were performed with the two gaps positioned on either side or the top and bottom, as shown in Figure 4.11, in order to visualize the asymmetric cloud dispersion.

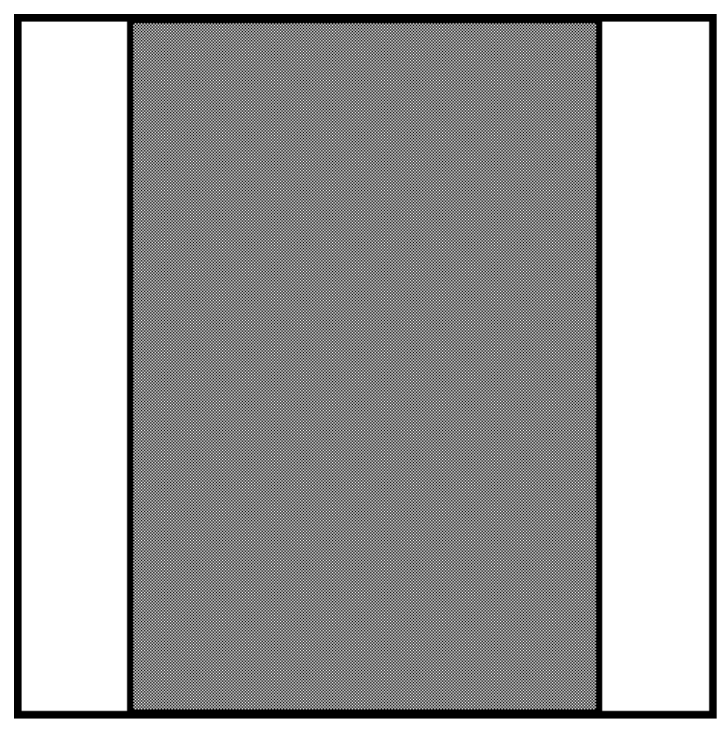

(a)

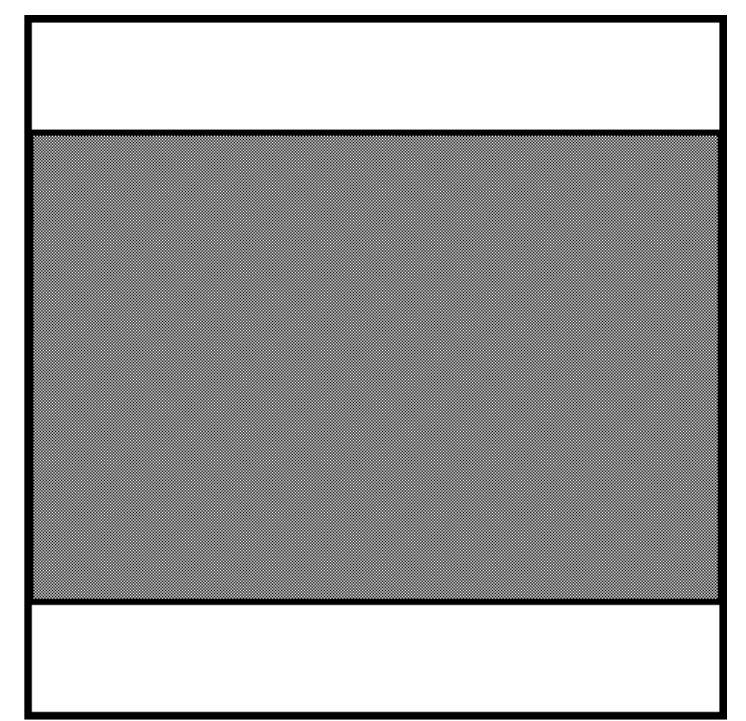

(b)

Figure 4.11: Modified particle wafer with $12.7 \mathrm{~mm}$ sidewall gaps a) vertically oriented and b) horizontally oriented

The results obtained with the wafer in the orientation shown in Figure 4.11a are provided in Figure 4.12. The images show a uniform cloud leading-edge, with a cloud density gradient in the stream-wise direction. The cloud leading-edge appears to be very diffuse with the density increasing towards the rear of the cloud. In the first three images a very thick shock is immediately observed following the shock-wafer interaction. This is in contrast with tests where 
the wafer fills the entire cross-section, see Figure 4.5, where a shock wave forms far downstream. The shock wave that is observed ahead of the cloud in Figure 4.12 is the incident shock wave that has bypassed the wafer through the cut-away edges. To confirm this, plan-view tests were performed with the wafer oriented as shown in Figure 4.11b. The images captured with this different wafer orientation shown in Figure 4.13 shows a much more complex shock wave structure and cloud particle distribution. The diffracted shock waves from either side of the wafer reflect at the centerline of the channel producing a Mach stem, seen at $0.907 \mathrm{~ms}$ in Figure 4.13. The Mach stem grows with time as the two triple-points move in opposite directions. The shear layers originating at the two triple-points interact at the centerline of the channel. The shock wave structure develops over time where the transverse waves decay and the shock front becomes more planar. As the incident shock wave travels through the cut-away gaps the shear at the wafer edges strip away particles from the wafer. The stripped particles accumulate forming an elongated cloud in the recirculation downstream from the wafer. The side and plan view images in Figure 4.12 and Figure 4.13 are combined into a series of composite images in Figure 4.14, giving a full three-dimensional view of the development of the complex shock wave structure and the trailing particle cloud. The thick shock wave seen in the side view in Figure 4.12 is an integrated effect of the diffracted shock waves and the Mach stems. Furthermore, the planar cloud leading-edge that appears in Figure 4.12 masks the highly non-uniform particle cloud that forms downstream of the wafer that is observed in Figure 4.13. 

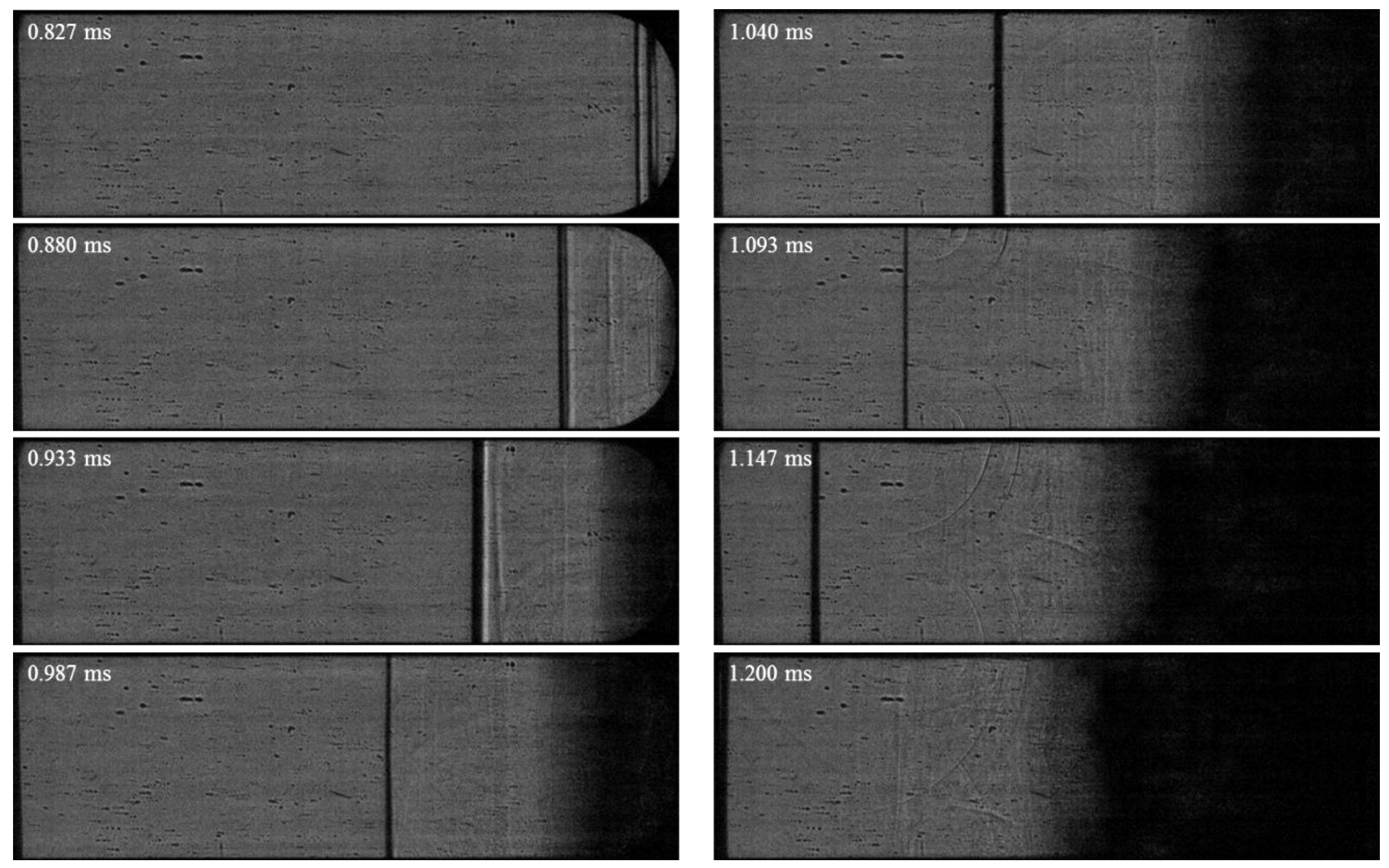

Figure 4.12: Images showing the shock wave and particle cloud dispersion. Results for 11 bar abs. driver pressure with 12.7 mm gap on sidewall wafer edges as shown in Figure 4.11a 

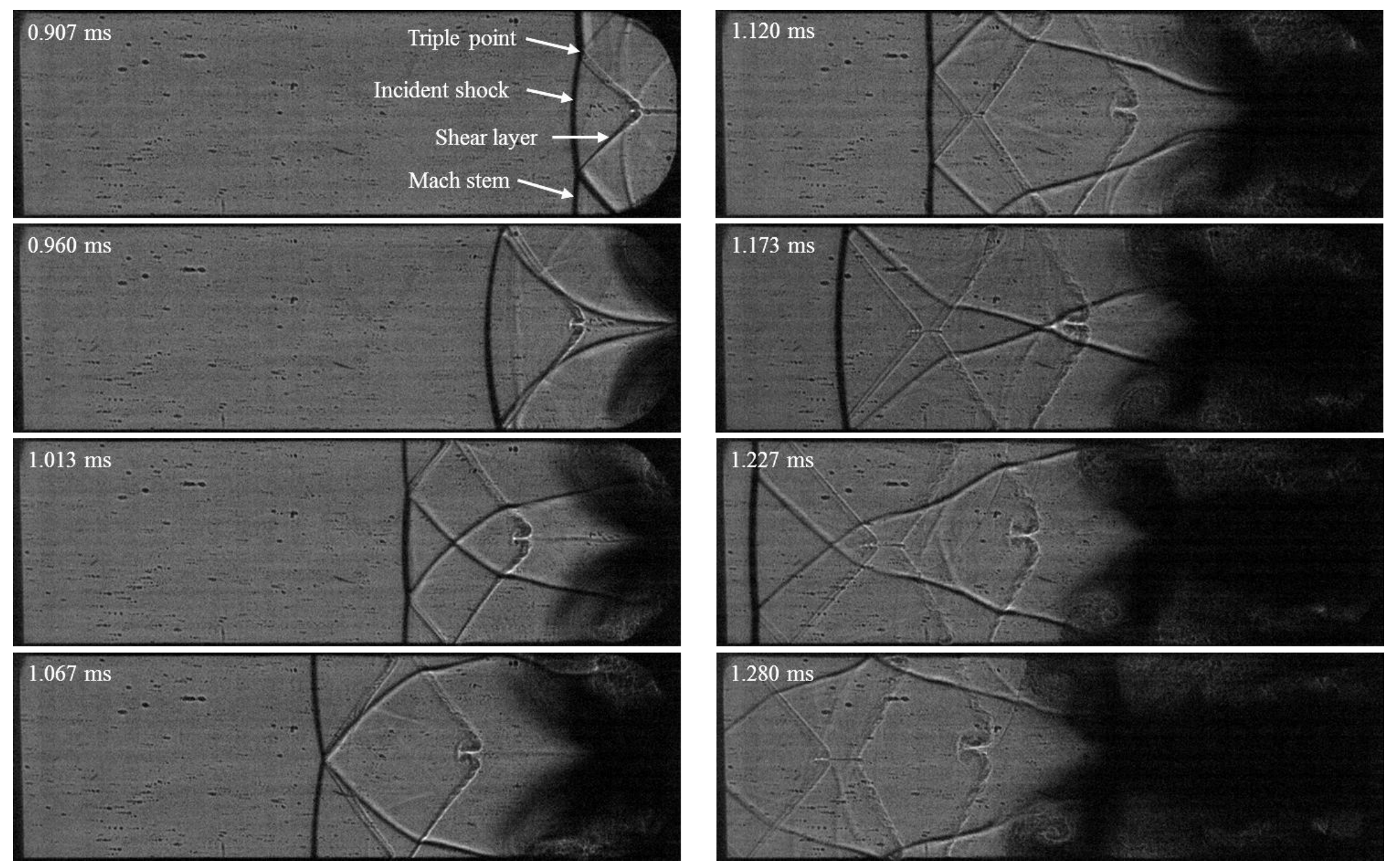

Figure 4.13: Images showing the complex shock wave structure and particle cloud dispersion. Results for 11 bar abs. driver pressure with $12.7 \mathrm{~mm}$ gap on top and bottom wafer edges as shown in Figure 4.11b 

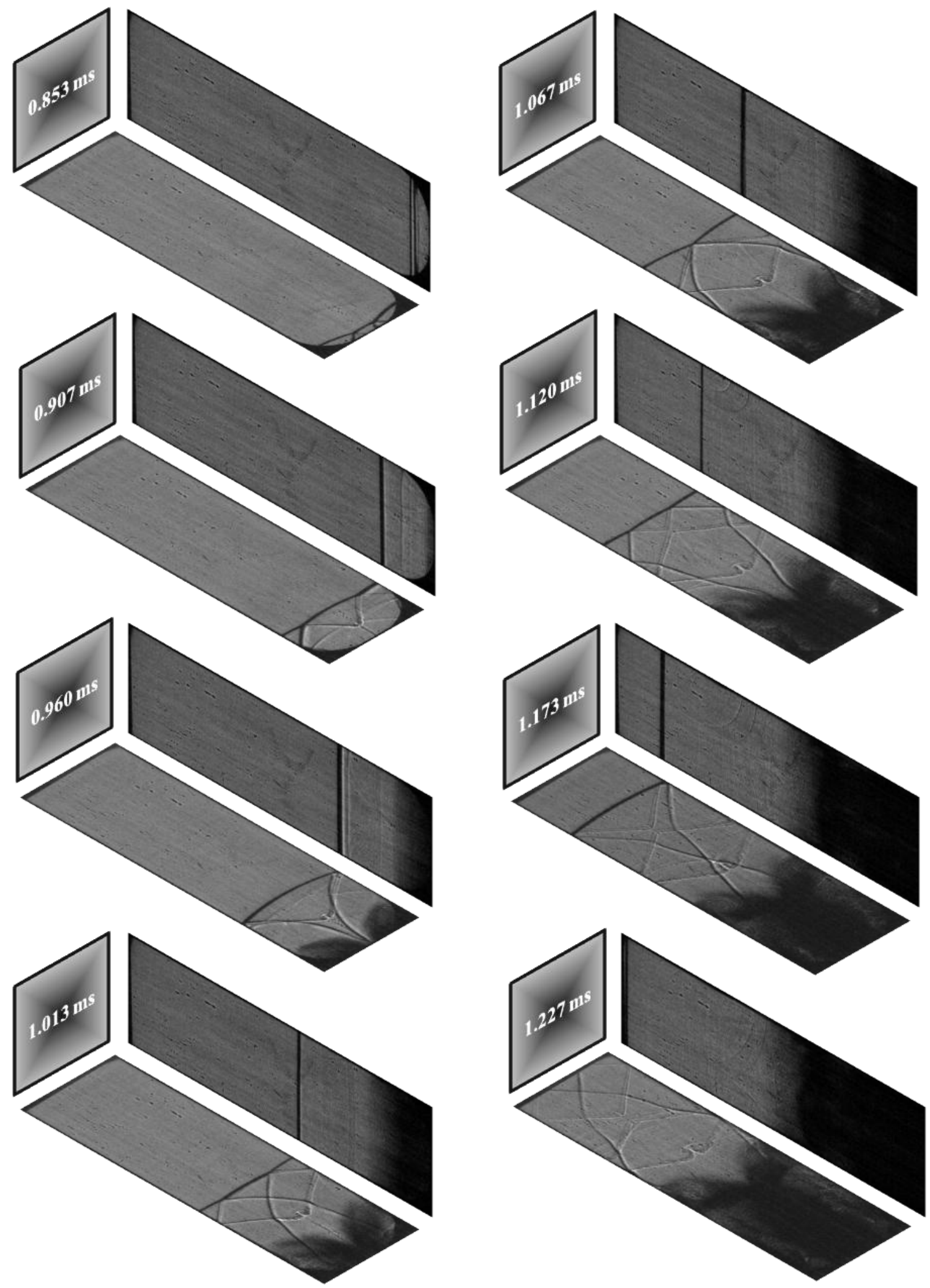

Figure 4.14: Composite image constructed from Figure 4.12 and Figure 4.13 
Since the wafer could not be placed in the field-of-view, the shock diffraction and early-time development of the wafer could not be visualized. In order to capture the early stage phenomenon, a Delrin ${ }^{\circledR}$ block of the same mass as the particle wafer was placed within the field of view, see Figure 4.15. A layer of alumina powder was placed on the top edge of the block in order to determine the dispersion pattern of the particles sheared off the edge of the actual wafer. The images in Figure 4.15 clearly show that there is no transmitted shock that passes through the block. In fact, over the $0.17 \mathrm{~ms}$ time elapsed for all the early time images in Figure 4.15 , the block does not move at all. The incident shock wave propagates through the gaps between the block edge and the channel side wall. The shock wave diffracts around the block's two trailingedges. The diffracted shock waves collide at the centerline at roughly $0.906 \mathrm{~ms}$ and proceed to form a roughly planar leading shock wave by $1.147 \mathrm{~ms}$. The shock diffraction also forms a vortex that travels toward the channel centerline. The two vortices and the trailing shear layer eventually merge to form a turbulent recirculation zone behind the block. The powder on the top-edge of the block is very quickly mobilized, forming an elongated cloud that initially follows the shear layer, see the image at $0.987 \mathrm{~ms}$. Over time, the cloud disperses and accumulates in the recirculation zone, see the image at $1.947 \mathrm{~ms}$. 

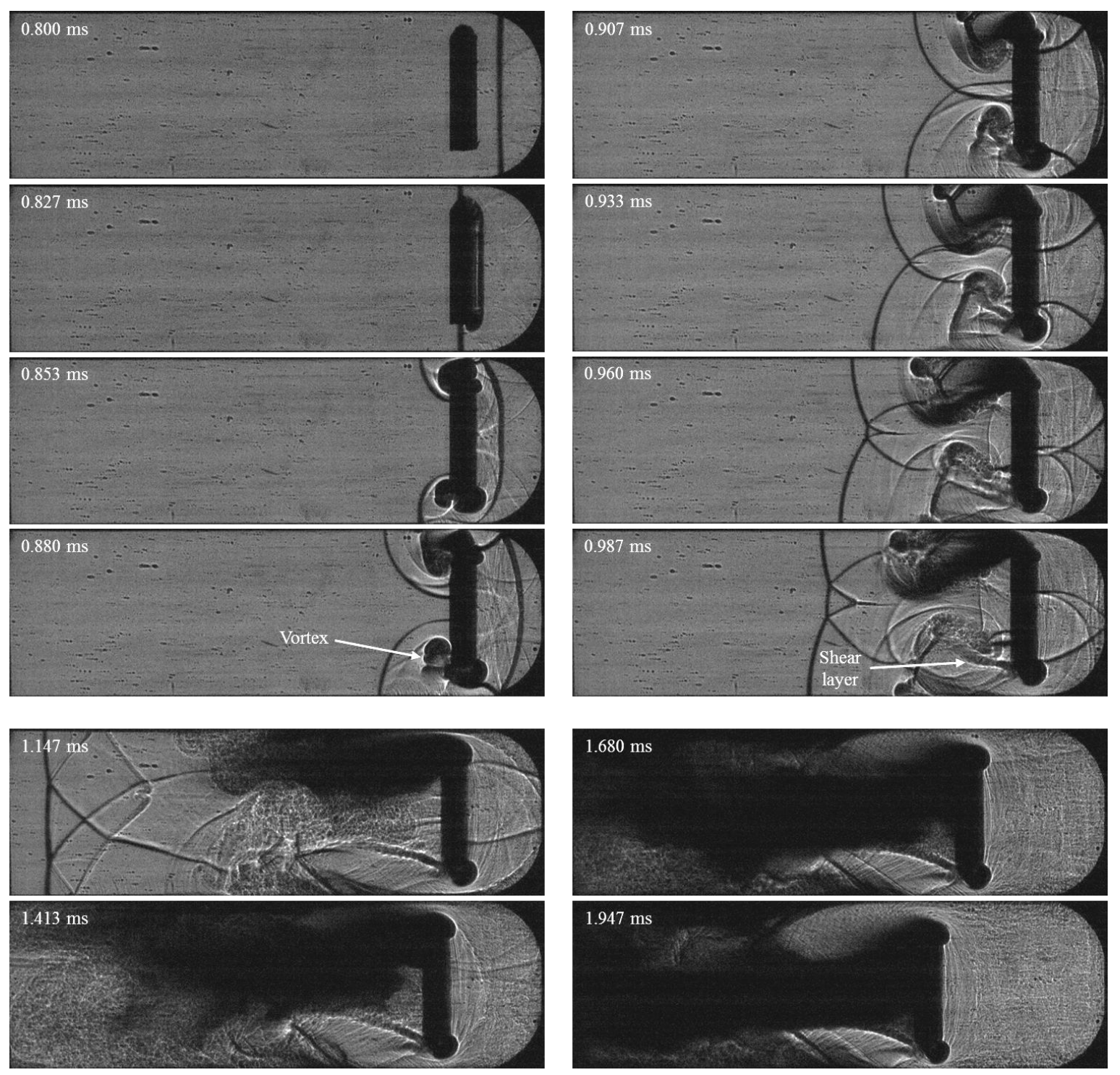

Figure 4.15: Images showing shock and particle dispersion from the top of a Delrin ${ }^{\circledR}$ block following the interaction of shock wave produced by 11 bar abs. driver (12.7 mm gap on top and bottom)

The results from Figure 4.12 to Figure 4.15 give a clear picture of what is happening if the wafer sample has gaps on the side walls. The pressure trace corresponding to the test shown in Figure 4.12 can be seen in Figure 4.16. 


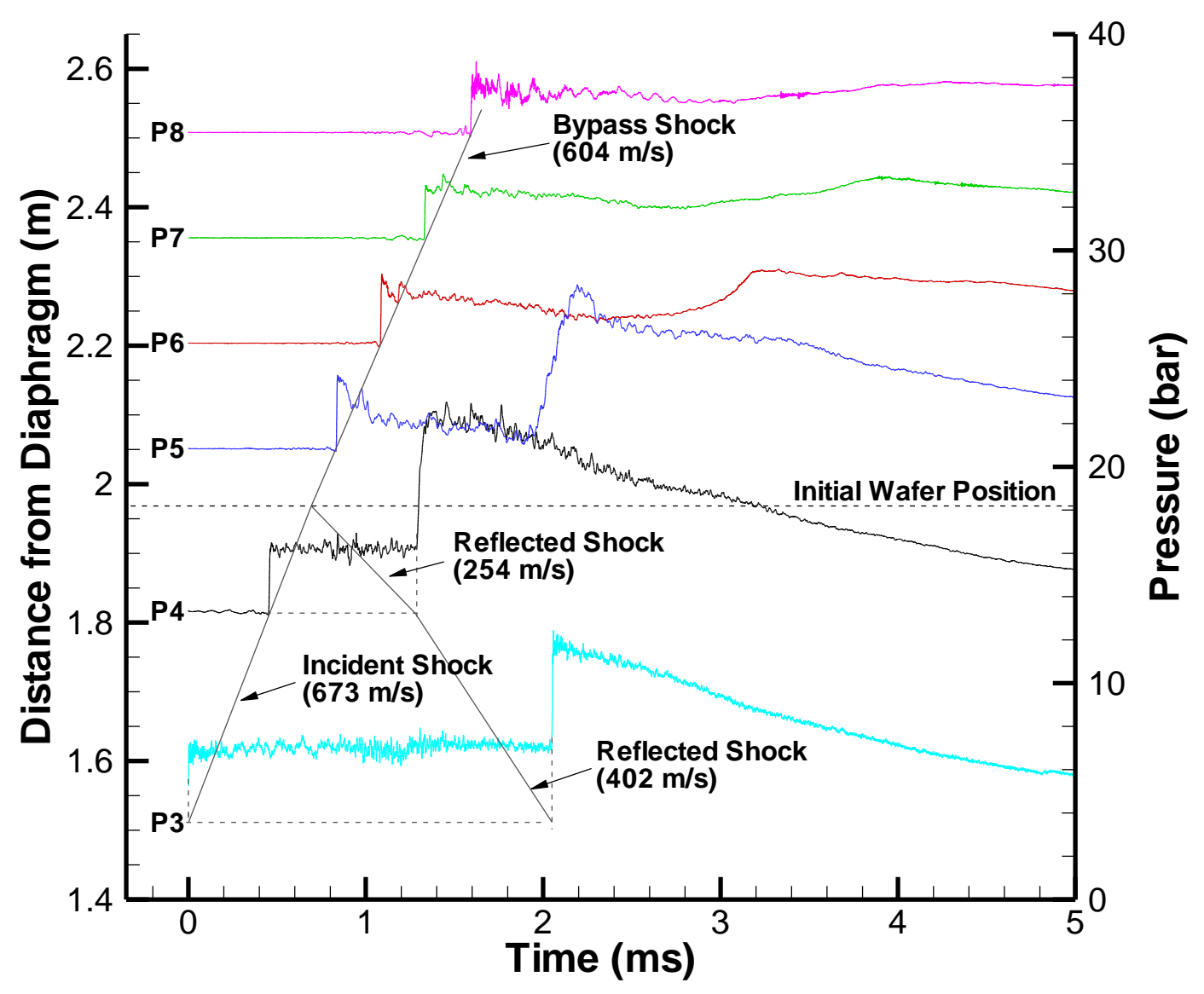

Figure 4.16: Results for 11 bar abs. driver pressure with $12.7 \mathrm{~mm}$ gap on sidewall wafer edges

The incident shock bypassing the particle wafer is shown clearly in Figure 4.16. The incident shock wave velocity of $673 \mathrm{~m} / \mathrm{s}$ is only slightly higher than the measured value of $604 \mathrm{~m} / \mathrm{s}$ for the bypassed shock wave. Shock pressures before and after the shock-block interaction are consistent at approximately 3 bar. At P5, the shock wave passing through the gap diffracts around the corner, forming an expansion behind the shock wave that brings the pressure down. The diffracted shocks coming through each gap then collide at the centerline, forming reflected waves, which cause the pressure pulse seen in P5 at approximately $2 \mathrm{~ms}$ in Figure 4.16. This expansion effect is local to the gaps at the wafer, as the reflected shocks eventually smooth the 
pressure, as can be seen as the pressure fluctuations decay downstream and the bypassed shock pressure is held.

\subsection{Downstream Surface Visualization}

High-speed video of the wafer downstream-surface was used to investigate the source of the nonuniform cloud leading-edge development. Moving the camera to approximately a $30^{\circ}$ angle from the shock tube axis allowed the observation of the front surface of the wafer during the shockwafer interaction. The interaction is observed in the front-lit direct video images shown in Figure 4.17. The development of the non-uniform leading-edge of the particle cloud, observed from the side-view videos in Figure 4.5, can now be seen in three-dimensions. The smooth surface of the wafer before the shock reflection can be seen in the first image at $1.033 \mathrm{~ms}$. In the next image a perturbation develops around the wafer edge. The corners of the wafer start to degenerate and accelerate ahead of the wafer edge starting at $1.433 \mathrm{~ms}$. From this time on, it is clear that the particle cloud advances faster in the corners, clarifying the source of the non-uniform side-view images of the cloud in Figure 4.5. The center of the wafer remains intact through the initial acceleration and is obscured by the leading edge of the particle cloud starting at $1.900 \mathrm{~ms}$. As a result, it is not possible to observe the breakup of the center of the particle wafer. The vertical white sliver in Figure 4.17 is light reflected off the front window. 

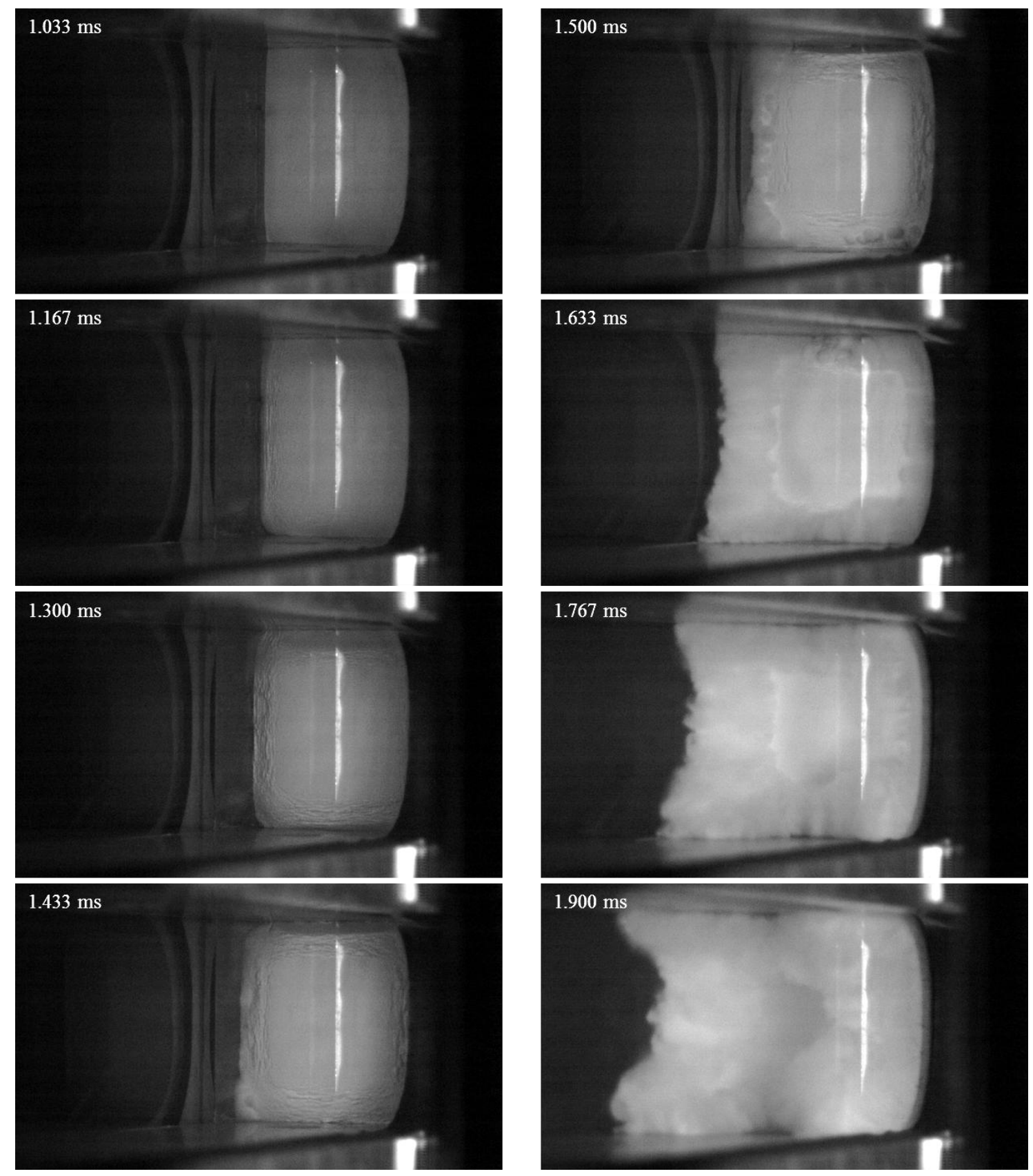

Figure 4.17: Images showing the initial wafer breakup and cloud development for 11 bar abs. driver pressure

Using an endplate with optical access enabled high-speed visualization of the wafer break up from a head-on perspective. The camera lens depth-of-field for this video was quite short with 
focus being lost after approximately $10 \mathrm{~cm}$. The advantage of the camera view shown in Figure 4.18 is that the wafer is visible in its entirety through the dispersion process.
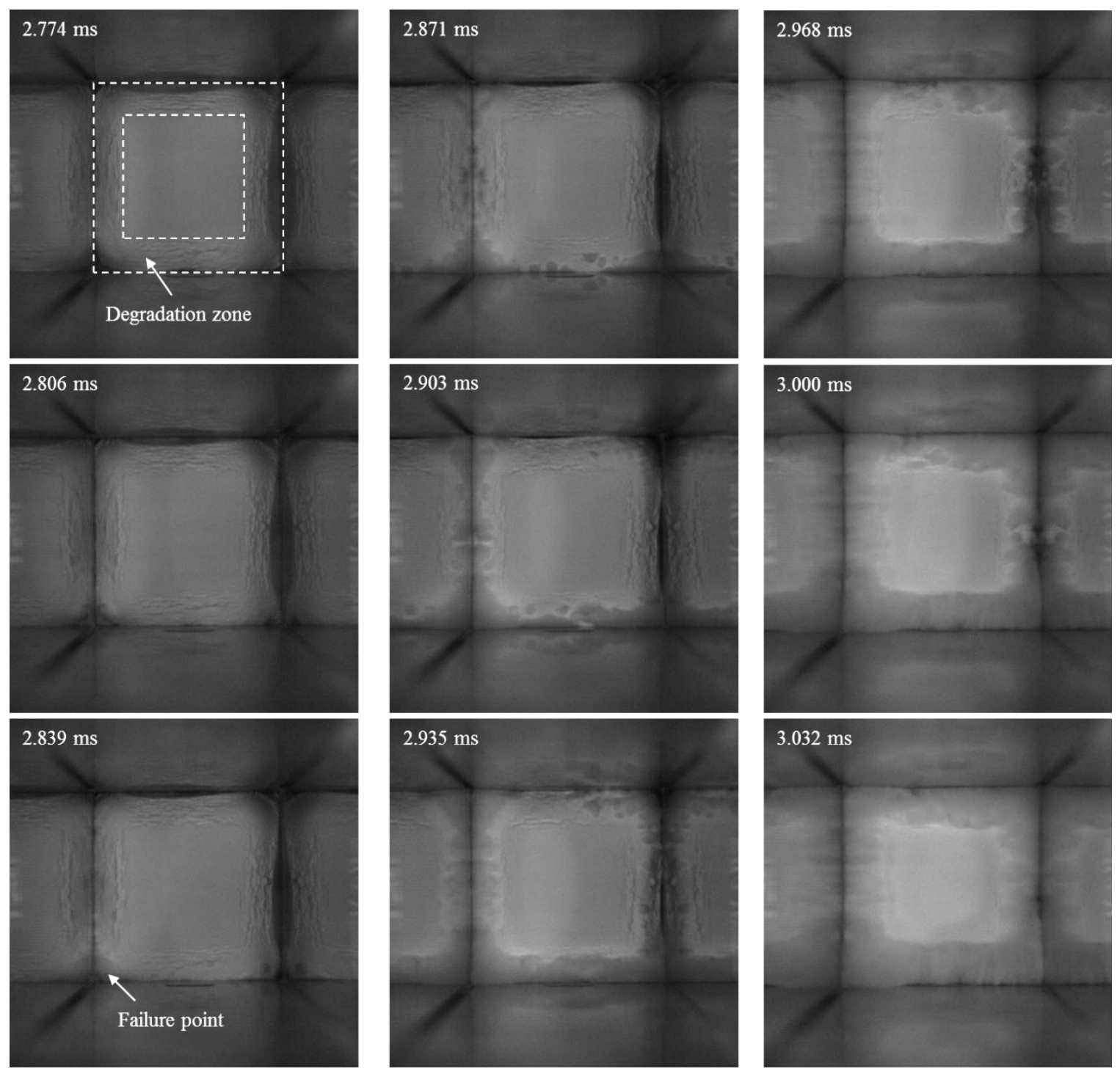

Figure 4.18: Head-on view showing initial wafer breakup with degradation zone and corner failure as well as cloud development for 11 bar abs. driver pressure

As the wafer advances, following shock reflection, the edges lag behind the center and ripples form along the edges. The ripples represent a degradation of the integrity of the particle wafer 
structure. The lateral extent of the degradation zone around the edge does not change with time. The center of the wafer remains intact as it advances and is eventually obscured by the dispersed particles from the degradation zone. The first signs of wafer failure appear in the corners as darker areas in the images in Figure 4.18. These dark areas correspond to particle jets that are first observed in the corners in Figure 4.17. Particle jets start to form on the left and bottom edges at $2.871 \mathrm{~ms}$, and then on the right and top edges at $2.935 \mathrm{~ms}$.

The advancement of the center of the wafer and the fixed edge boundary condition leads to shear along the edges of the wafer. The shear causes slip between the particles and the wafer degrades locally. As the wafer center advances, the level of shear decreases as the particle wafer edges break free from the fixed boundary condition. The square geometry produces shear concentrations in the corners, leading to the primary locations for wafer failure. As a result, the high pressure air produced by the shock reflection is able to penetrate the wafer locally at these locations, producing the particle jets observed in the corners. As the wafer advances, the shear along the edges increases leading to additional failure locations. Interestingly, the failure along the wafer edge does not occur uniformly, but instead occurs locally forming discrete particle jets. These jets associated with the failure of the wafer are analogous to the jets that form in largescale multiphase explosions $[8,10,17]$. In these large-scale experiments, the spherical expansion of the solid explosives does not generate shear in the surrounding packed particle bed shell, instead the outward radial motion results in a circumferential tension in the particle shell. Based on the failure mode observed in the present wafer tests, it is possible that discrete failure points in the shell are the origins of the high-speed jets that are observed later in time.

The plate and die method used to form the wafer causes internal stresses within the wafer that push against the inside edges of the plate. Since the loose powder is compressed vertically into a constant area die, particles will compress not only vertically, but horizontally as well. The 
axial pressure produced by the hydraulic press causes a lateral pressure to be produced as shown in Figure 4.19.
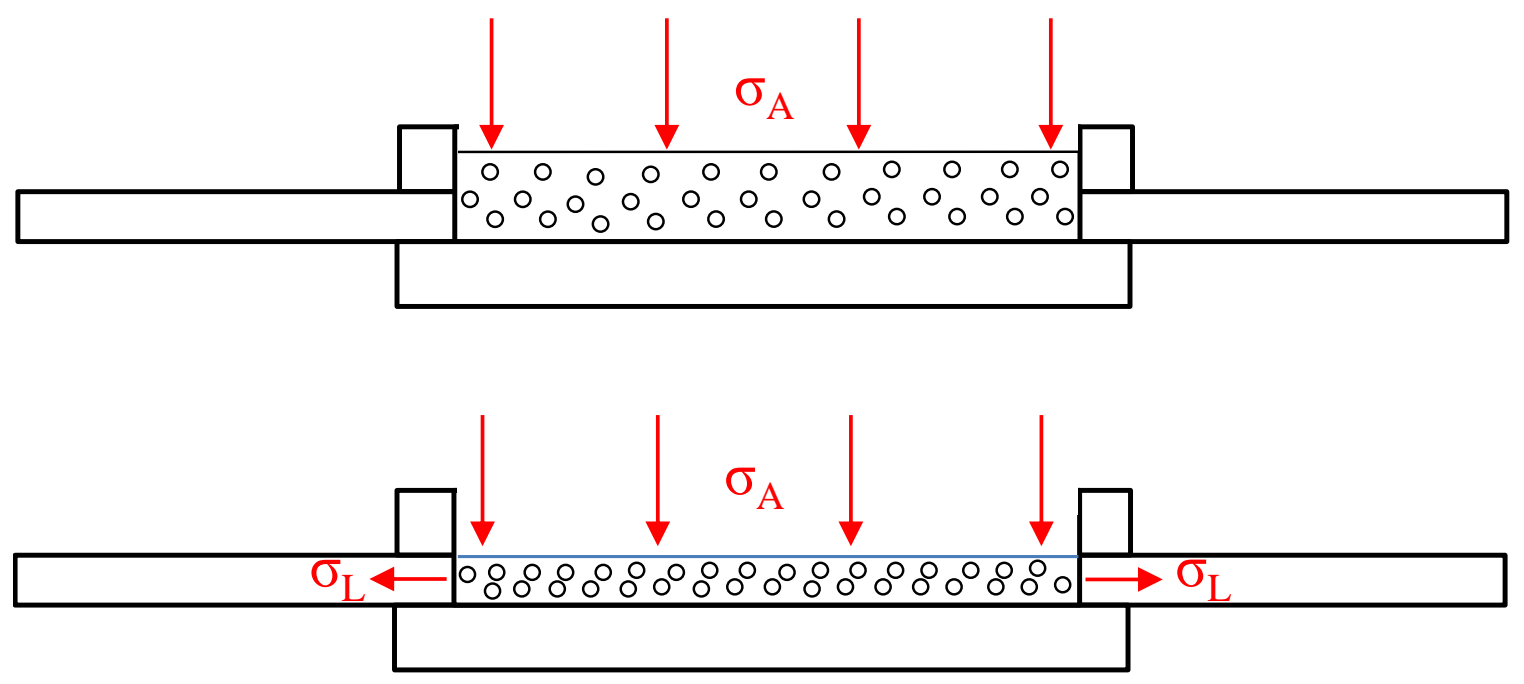

Figure 4.19: Powder within die before and after compaction showing forces on plate sidewalls once compressed

Once the plate is removed from the die, frictional forces between the particles hold them together on the wafer faces. On the wafer edges, there is also a frictional force between the particles and the plate side wall that is enhanced by the internal stress within the wafer. This will cause the wafer edges to resist shear against the plate sidewalls. At the corners, this effect becomes enhanced due to the close proximity of two edges. Since the corners of the wafer are held in place more rigidly due to the plate sidewall effects, the wafer stretching is greatest just inside of the corner, as seen in Figure 4.20. 


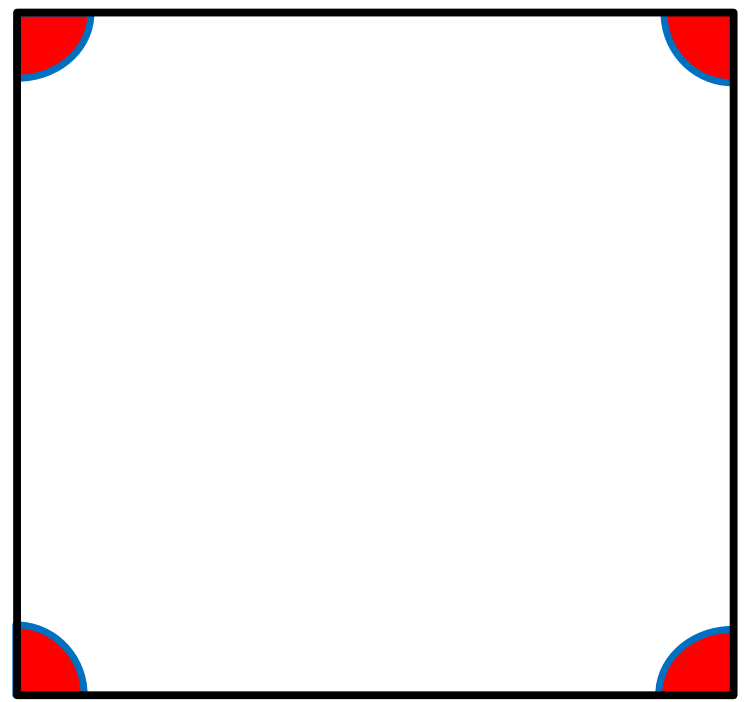

Figure 4.20: Particle wafer showing areas where movement is restricted due to plate sidewall effects in red and corner failure zones in blue

In the bottom-left corner at $2.774 \mathrm{~ms}$ in Figure 4.18, wafer movement it restricted all the way in the corner, causing failure and allowing high pressure reflected gas to permeate through. This is the cause of the local corner acceleration and the "V" shape of the cloud from the side view.

\subsection{Particle Wafer - Circular Cross-section}

In order to eliminate particle wafer corner effects, experiments were carried out in a circular geometry. This was done by inserting tightly fitting $7.6 \mathrm{~cm}$ outer-diameter, $7.0 \mathrm{~cm}$ inner-diameter acrylic tubes into the channel on either side of the wafer. Due to light refraction through the round tube, shadowgraph images were not able to be obtained. In addition, the inserted tube also blocked the pressure transducers, so no pressure data was available for this geometry. The same wafer loading technique shown in Figure 3.6 was used, so the wafer characteristics are identical to the square channel experiments with no tube. Images from this test are shown in Figure 4.21. 

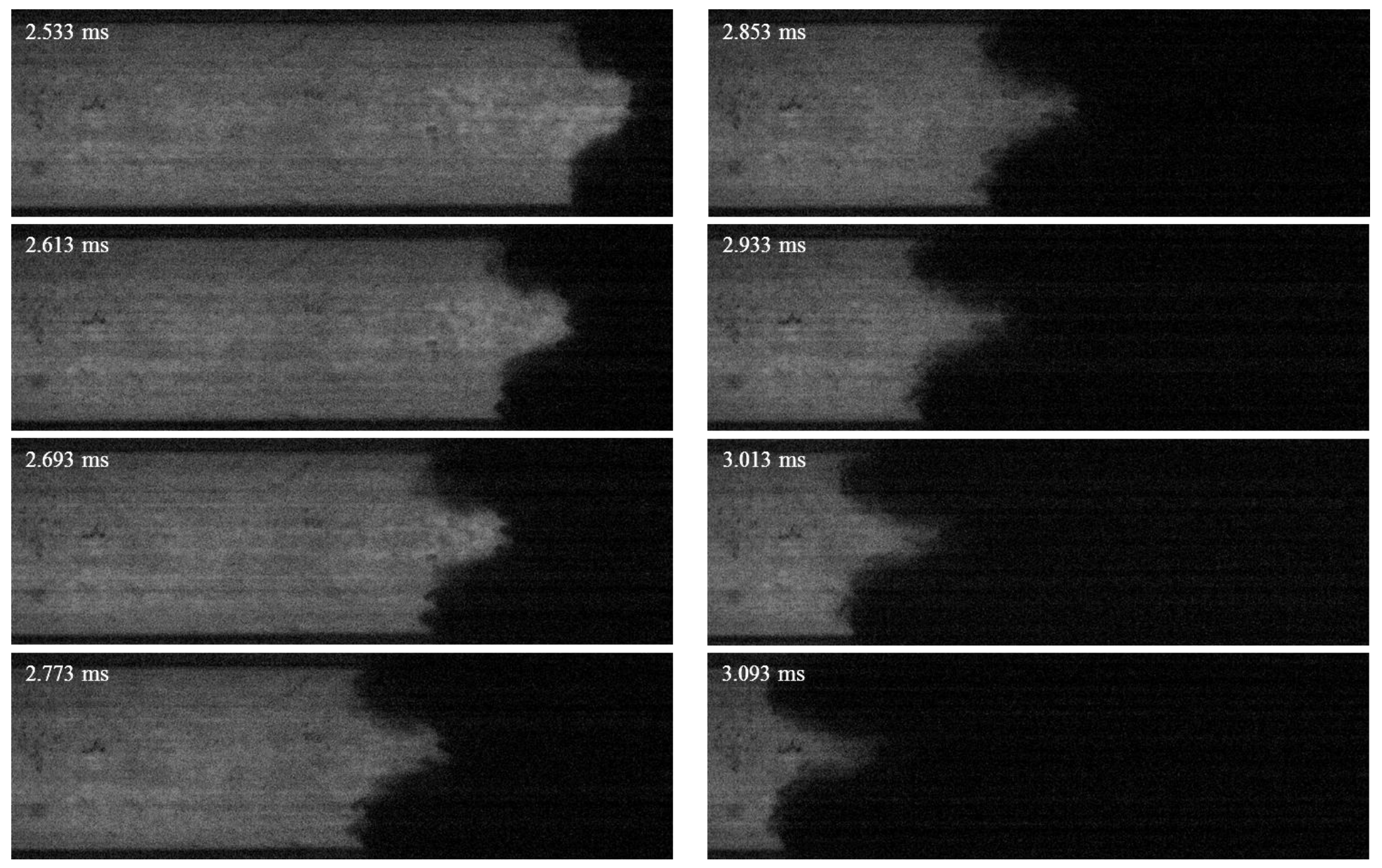

Figure 4.21: Side-view of 11 bar abs. driver pressure round cross-section 
Results from the round cross-section reveal the same "V" shaped cloud front that was present in the square channel tests. Based on the video, the cloud front velocity was measured to be $294 \mathrm{~m} / \mathrm{s}$. This is close to the $319 \mathrm{~m} / \mathrm{s}$ cloud front velocity that was measured in the square channel tests. Taking a similar approach as with the square channel, a Delrin ${ }^{\circledR}$ block of the same mass as the particle wafer was placed inside the round cross-section. The measured velocity of the Delrin ${ }^{\circledR}$ block averaged $160 \mathrm{~m} / \mathrm{s}$ in the round cross-section, which matched the $160 \mathrm{~m} / \mathrm{s}$ average block velocity measured in the square cross-section tests.

The head-on view of the wafer failure in this round geometry is shown in Figure 4.22. The round black line is the tube inner diameter and the disturbance outside this line is a reflection of light off the inner surface of the tube. Note the $7.0 \mathrm{~cm}$ inner-diameter of the tube is smaller than the $7.6 \mathrm{~cm}$ square wafer, so the shock wave punches a circular section out of the full square wafer. Therefore, the circular tube tests have a different wafer boundary condition than the square channel experiments where the wafer edge lines up with the channel inner-walls. Similar to what was observed in the square channel tests, a degradation zone develops around the edge of the circular wafer. In this geometry, the local failure points, where the particle jets form, are uniformly distributed along the wafer circumference, see the $2.742 \mathrm{~ms}$ image in Figure 4.22 . This is different from the square channel tests where the failure points are primarily inside from the channel circumference, see images in Figure 4.18 at $2.903 \mathrm{~ms}$ and $2.968 \mathrm{~ms}$. 

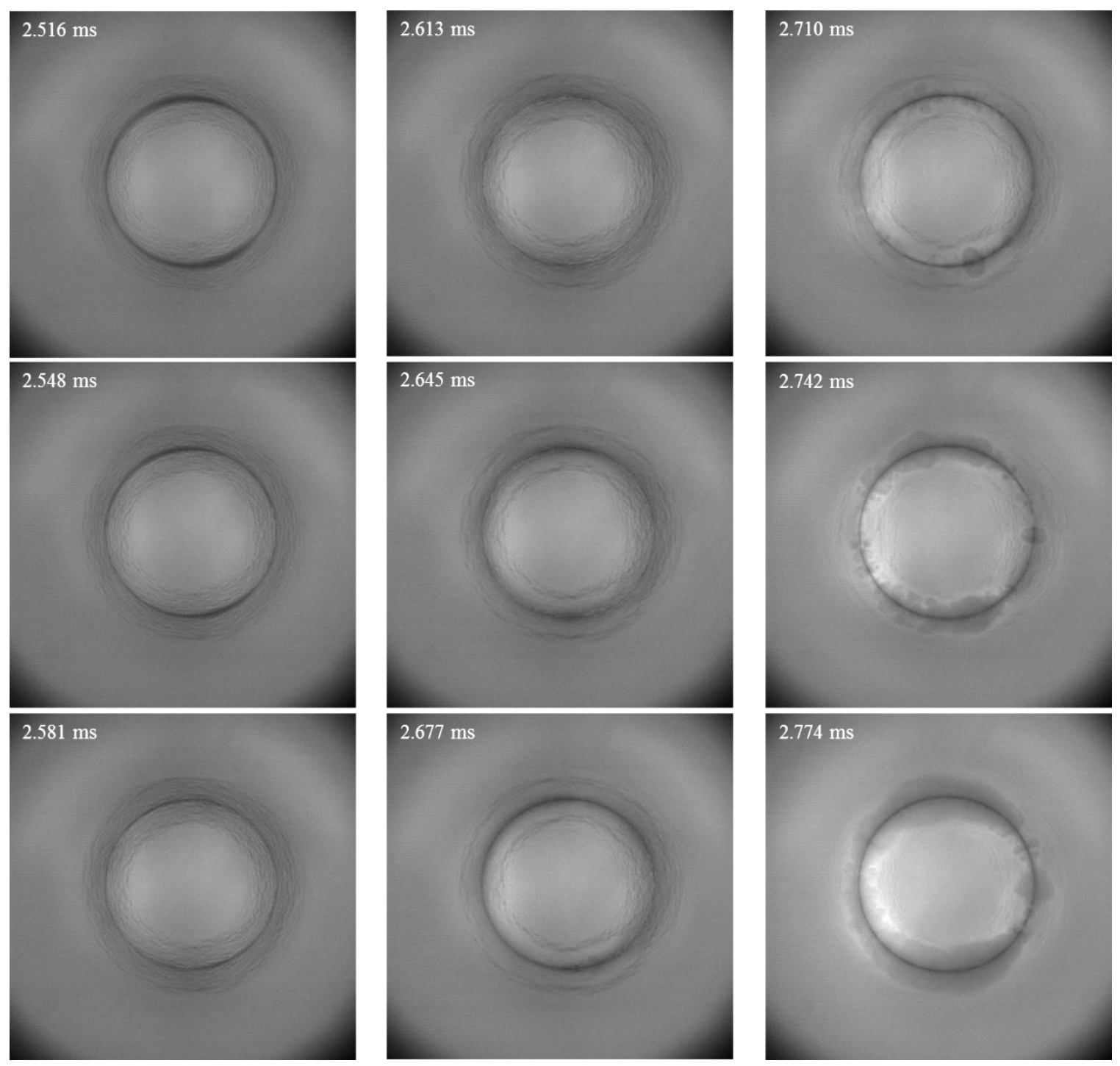

Figure 4.22: Head-on view showing initial wafer breakup and cloud development in a circular tube for 11 bar abs. driver pressure

With the circular tube, it was possible to push a circular particle wafer into the tube at the beginning of the optical section using the tool described in Section 3.3. In this geometry the wafer is not compressed up against the side wall, as was the case in the square channel tests where the die is inserted between the flanges. A series of end-view images from a test is provided in Figure 4.23. The free edges results in a larger degradation zone around the circumference, leaving a 
smaller diameter portion of the wafer intact. Particle jets form primarily along the tube inner-wall but some failure points form away from the tube wall. Overall, the failure mode between the plate-mounted and the pushed-in wafers are very similar.
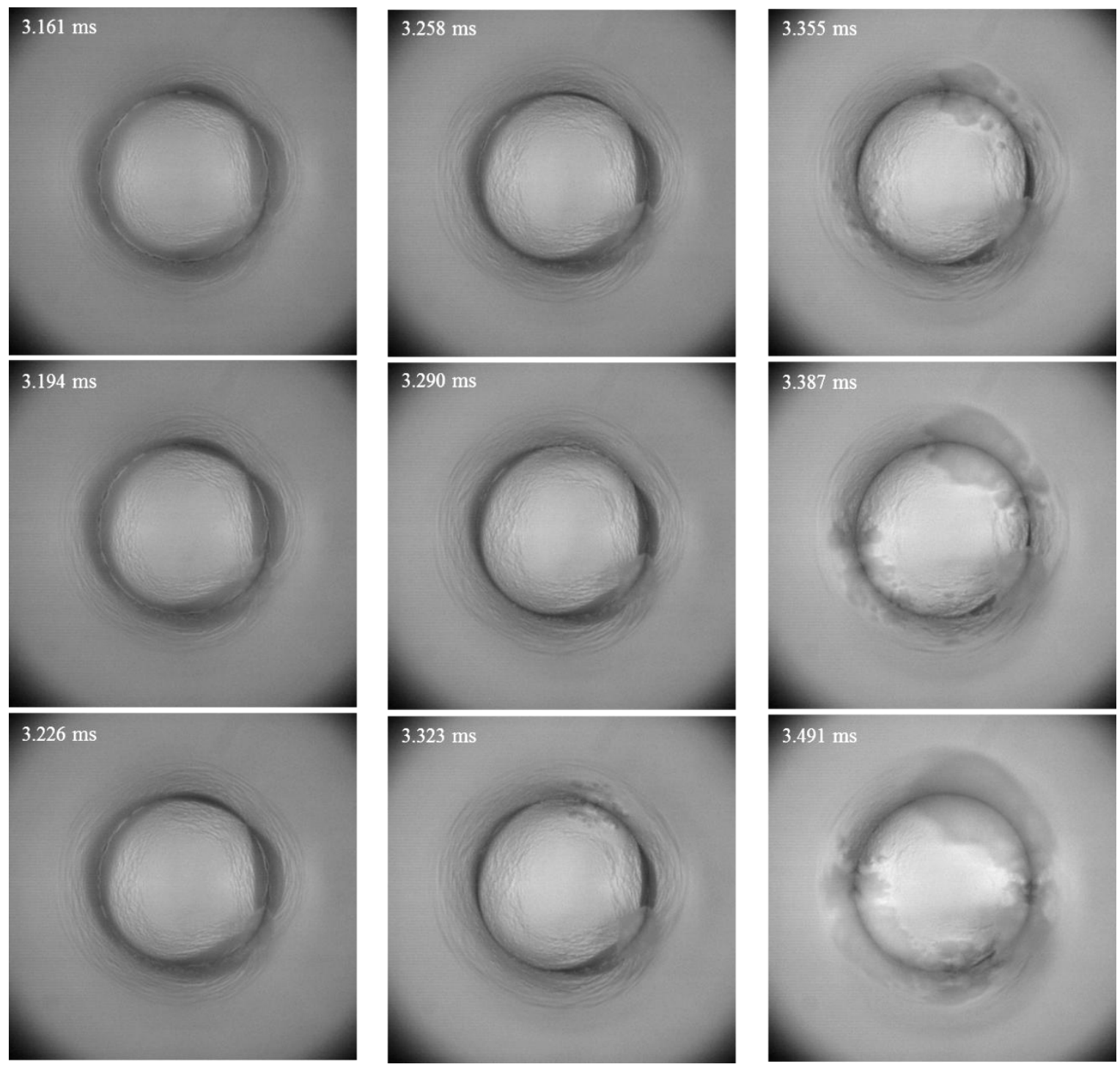

Figure 4.23: Head-on view for 11 bar abs. driver pressure showing initial wafer breakup in round cross-section with a pushed-in particle wafer 
Images from the side view video corresponding to the pushed-in wafer test from Figure 4.23 is shown in Figure 4.24. The cloud front shape in this case appears to be much more uniform and the inverted "V" shape no longer persists. 

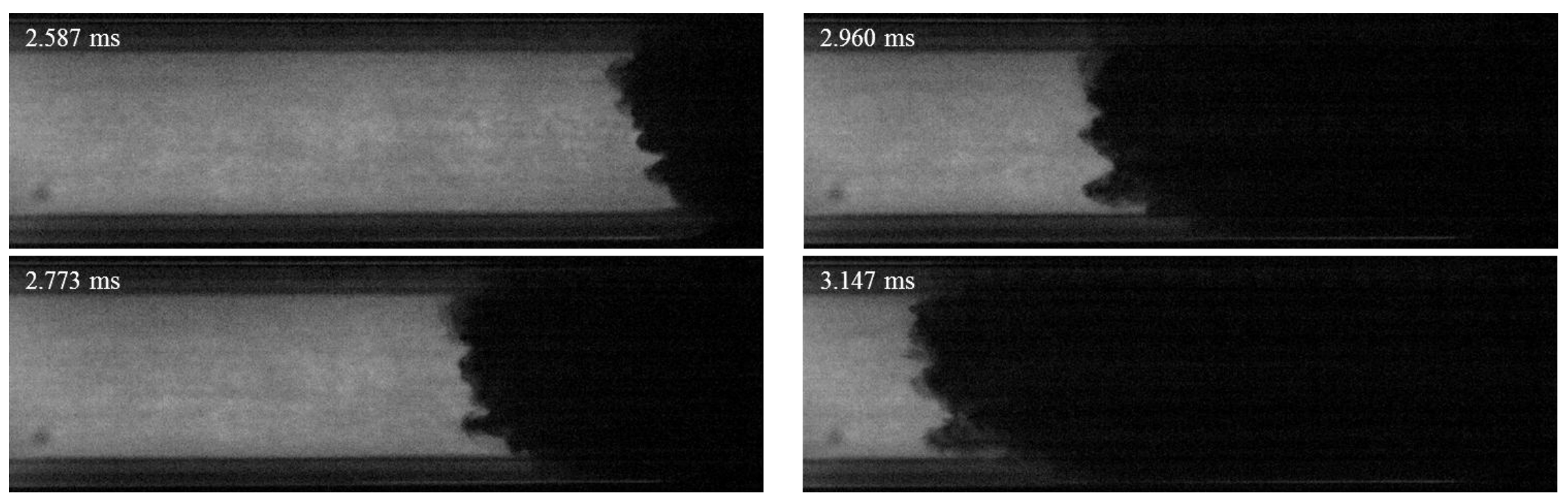

Figure 4.24: Particle cloud leading-edge for pushed-in wafer in round cross-section. From tests with 11 bar abs. helium driver 
The particle cloud leading-edge velocity obtained from side-view, back-lit regular video is shown in Figure 4.25. The data shown in Figure 4.25 is taken from a typical test of each configuration. As can be seen there is considerable scatter in the circular tube data but in general the cloud leading-edge velocity is similar for the two wafer mounting methods. The cloud leading-edge velocity for the square channel lies roughly $50 \mathrm{~m} / \mathrm{s}$ higher than that observed in the circular tube. This data shows that the initial rate of acceleration over the first $8.5 \mathrm{~cm}$, outside the camera fieldof-view, is faster in the square channel resulting in a higher velocity at the start of the field-ofview. However, within the field-of-view the rate of acceleration in both geometries is similar.

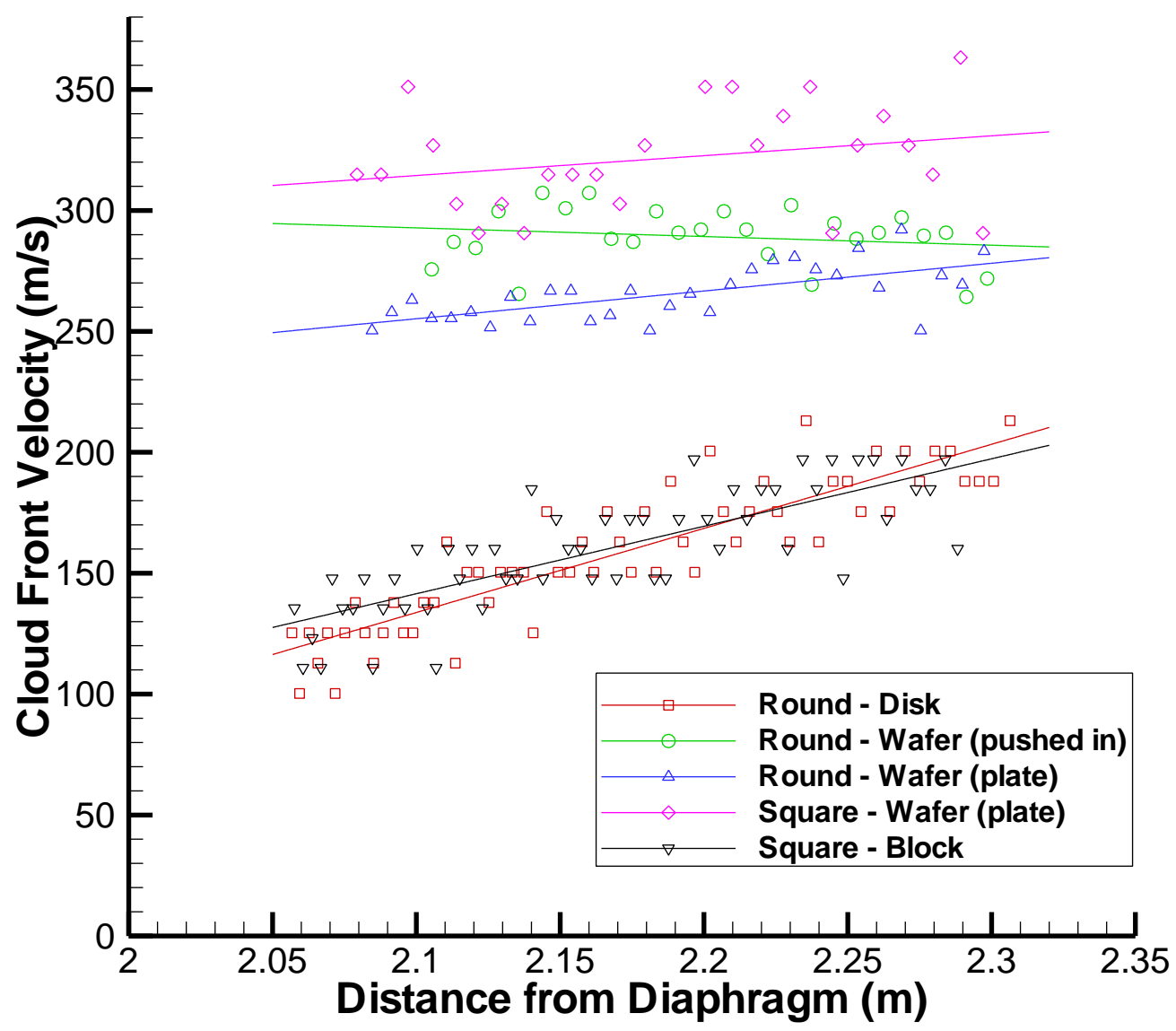

Figure 4.25: Experimental cloud front velocities from square and round cross-section 
Since the pressure behind the wafer and the mass per unit area is the same, the difference in the acceleration over the first $8.5 \mathrm{~cm}$ can be attributed to the difference in the friction produced by the wafer edge degradation in the square and circular geometry. Taking a similar approach as with the square channel, a Delrin ${ }^{\circledR}$ disk of the same mass as the particle wafer was placed inside the tube. As seen in Figure 4.25, the velocity of the square block and circular disk is almost identical and is lower than the particle wafer cloud leading-edge. The very different velocity history between the cloud leading-edge and the Delrin ${ }^{\circledR}$ block and disk is due to the failing of the wafer edges. The gas that first jets through the wafer quickly accelerates particles torn off the edges downstream. Therefore the high-speed dispersed particles constitute the leading-edge of the cloud. Since the trailing-edge of the cloud cannot be detected in the videos, it is not possible to know the velocity of the intact central part of the accelerating wafer. It is very likely that the intact wafer core velocity is close to the slower block/disk velocity observed in Figure 4.25. The scatter within tests can be attributed to the very small time step (camera frame rate) and the stochastic nature of the rolling and billowing cloud leading edge.

\subsection{Particle Cloud Formation Mechanism}

It is clear that the dispersion of a particle cloud with a high initial particle volume fraction $\left(\varphi_{\mathrm{p}}=\right.$ 0.48 for the wafer used in the present tests) is a multi-dimensional phenomenon. This is in contrast with the dispersion of a much lower $\varphi_{p}=0.21$ initial particle volume fraction cloud in the experiments performed by Wagner et al. [12]. At such a low particle volume fraction, the cloud consists primarily of gas and thus is relatively compressible compared to the wafer that is precompressed to 254 bar in a hydraulic press. As a result, in the Wagner et al. [12] particle curtain tests, the shock wave propagates through the curtain producing a dispersing cloud. The cloud dispersion occurs because the leading and trailing edges of the particle curtain can propagate at different velocities in response to the interaction of the incident shock wave with the leadingedge, and the transmitted shock wave with the trailing edge. For the wafer, the Mach 2 incident 
shock wave is not able to compress the wafer and thus a shock wave does not propagate through the wafer. Instead, the wafer is accelerated uniformly and is dispersed by the failure of the wafer due to shear resulting from the boundary conditions. The wafer behaves much like a solid, so in order to have the leading and trailing edge of the wafer propagate at different velocities a shock pressure larger than the wafer pre-compression pressure is required. A stronger incident shock would accelerate the wafer leading-edge and the transmitted compression stress wave would reflect at the trailing-edge as a tensile stress wave. If the tension is higher than the effective wafer yield stress, a spallation process would ensue where particles would detach from the wafer trailing-edge. This spallation process would result in one-dimensional particle dispersion and edge boundary conditions would play a less important role in the wafer breakup, compared to that observed in the present experiments using a weak incident shock wave. Testing with stronger shock waves is not possible in the present shock channel due to the limiting strength of the glass windows.

It is important to note that the trailing-edge of the wafer is not visible from the side-view videos, since it is quickly obscured by the bypassing particles. Based on the end-view videos, the centre of the wafer remains intact until it is obscured by edge particle coming in front of it. If spalling were to occur, it would be on a very short time-scale and it would be visible immediately after the shock-particle wafer interaction. Therefore, it is suspected that the wafer remains intact after the shock interaction and then is slowly eroded by the flow around the wafer.

\subsection{Increased Cloud Velocity}

In an effort to more closely model explosive dispersion events, attempts were made to increase the particle cloud velocity. It was important that the method used not involve going to a higher driver pressure, as the maximum safe drive pressure had already been reached. Two methods were used for this: a low pressure driven section and an area contraction to accelerate the flow. 


\subsubsection{Low Pressure Driven Section}

As described in Section 3.2.4, the shock tube used in the present experiments had the ability to use a low pressure driven section. By vacuuming the driven section, a higher pressure ratio was achievable across the diaphragm while maintaining a driver pressure of $11 \mathrm{bar}$. Adjustments were made to the inner-diaphragm volume fill procedure to account for the new pressure differences across them. Using the square wafer, a series of tests were performed with the driven section at 0.5 bar absolute to see if a higher cloud velocity was achievable. A typical pressure history is shown in Figure 4.26. 


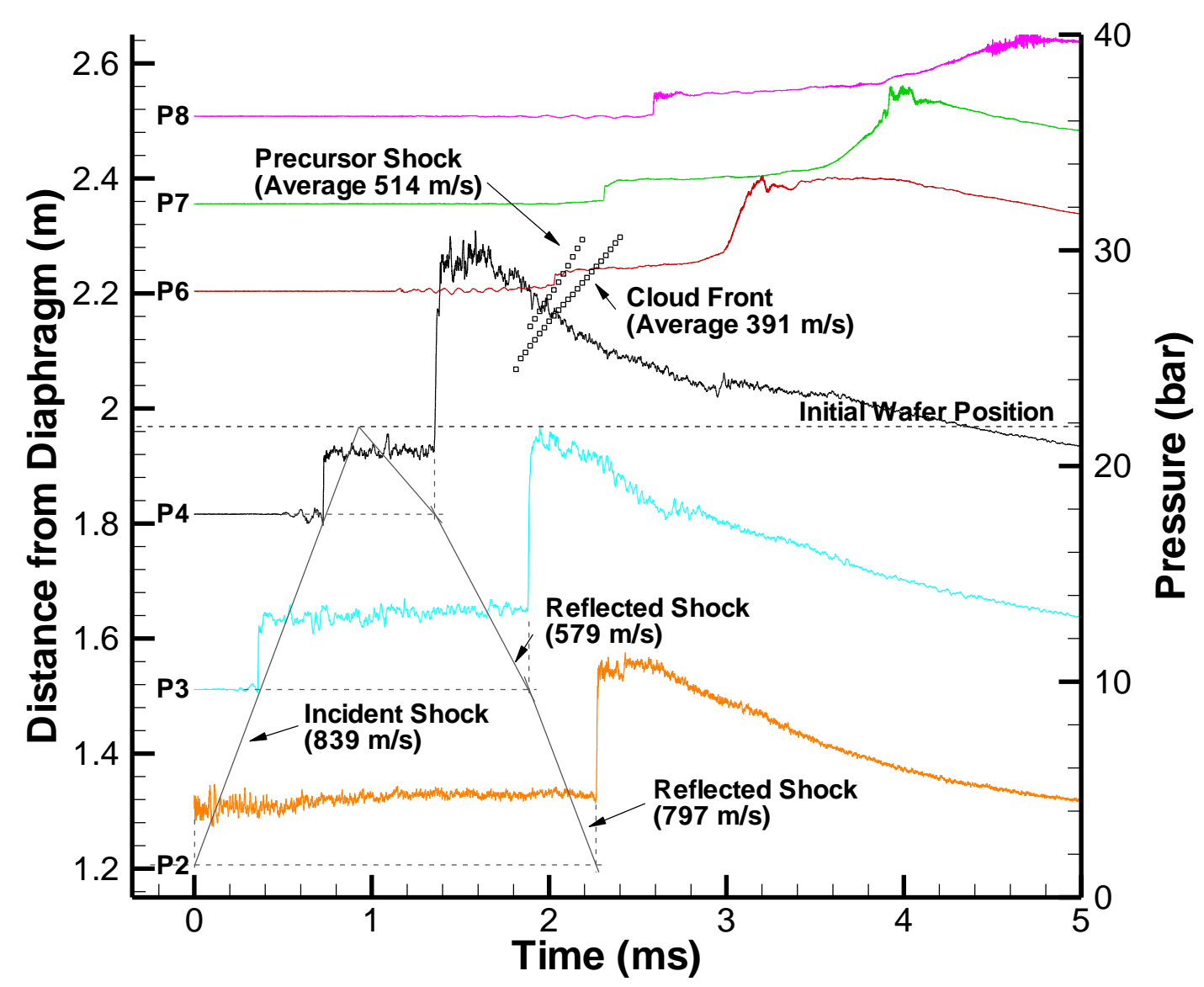

Figure 4.26: Results for 11 bar abs. helium driver pressure with 0.5 bar abs. atmospheric air driven section pressure

The incident shock velocity of $839 \mathrm{~m} / \mathrm{s}$ (Mach 2.4) was significantly faster than that obtained running the driven section at 1 bar that produced roughly a $700 \mathrm{~m} / \mathrm{s}$ (Mach 2.0) incident shock wave. It can be seen that the compression waves downstream from the accelerating wafer coalesce much further upstream when compared to the experiments run with the driven section at atmospheric pressure. The cloud front velocity was also found to be faster, averaging $391 \mathrm{~m} / \mathrm{s}$, compared to the $320 \mathrm{~m} / \mathrm{s}$ average velocity produced by the Mach 2.0 shock wave. Although this produced a higher cloud front velocity, an explosive dispersal achieves particle velocities on the order of $1000 \mathrm{~m} / \mathrm{s}$, making the Mach 2.4 driven cloud fall well short of that target. Another issue 
with the low pressure driven section is that while a higher cloud velocity was able to be obtained, the low pressure particle propagation atmosphere also pulled the experiment away from real world explosive dispersal conditions where the medium the particles are travelling through is air at atmospheric conditions.

\subsubsection{Area Contraction}

Another method used to increase the cloud front velocity was to use an area contraction prior to the wafer to increase the shock wave, and associated flow, velocity in the shock tube driven section. To do this, two $2.54 \mathrm{~cm}$ by $7.62 \mathrm{~cm}$ aluminum ramps were attached to the top and bottom surfaces inside the optical section. The aspect ratio of the ramps gives an arbitrary angle of $18.4^{\circ}$ to the horizontal and a cross-sectional area ratio of 0.33 . Tests were initially run with a particle wafer placed behind the ramps, as seen in Figure 4.27. 

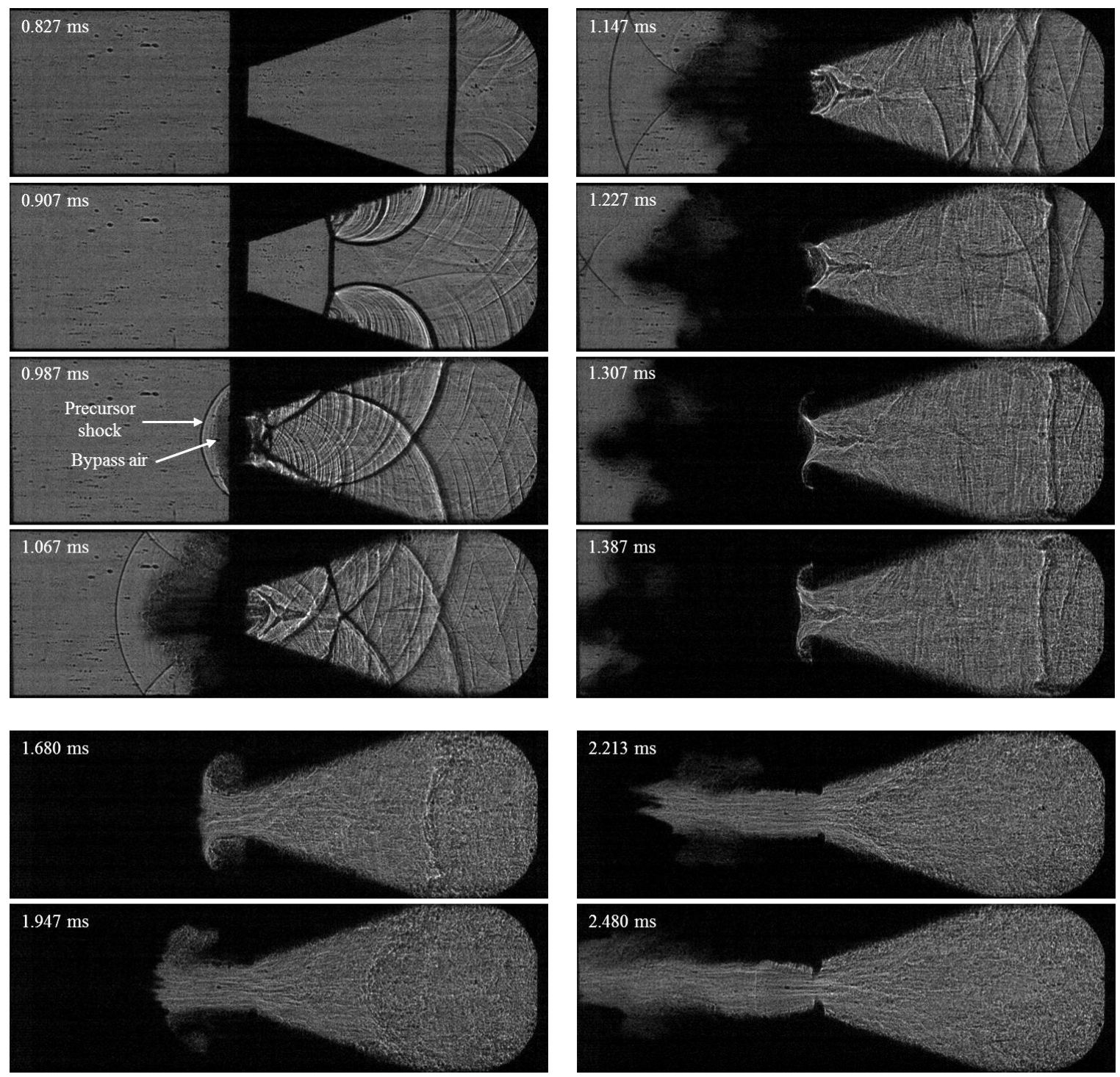

Figure 4.27: Images from 11 bar abs. helium driver with opposing $18.4^{\circ}$ ramps before particle wafer

In the first frame of Figure 4.27, an incident shock wave can be seen approaching the ramps, with a particle wafer placed behind the ramps, covering the entire $7.62 \mathrm{~cm}$ by $7.62 \mathrm{~cm}$ area of the shock tube driven section. At $0.907 \mathrm{~ms}$, the shock wave is seen travelling up the ramps, producing a Mach reflection configuration. This is because the angle of the ramps is too great for the reflected shock to turn the flow angle enough so that it is parallel with the ramps. The Mach 
stems can be seen travelling up the ramps, and the triple points converge at $0.987 \mathrm{~ms}$ when the particle wafer is impacted. A reflected wave is clearly seen propagating back upstream at 1.227 ms. The resulting wafer dispersion is much different than was observed in the open channel. A precursor shock wave is observed at $0.987 \mathrm{~ms}$ that is the result of small gaps that exist between the wafer and the glass side windows produced when the wafer is slid into position. Also evident in the same image is reflected shock compressed air that bypasses the side of the wafer. In the next image, at $1.067 \mathrm{~ms}$, the bypass air can be seen to convect particles that are sheared from the side of the wafer. The precursor shock and associated gas and particle flow is observed to be two-dimensional due to the change in duct area. As can be seen later it time at $1.680 \mathrm{~ms}$, much of the particle cloud is entrained in a recirculation zone on the downstream side of the ramps. Particles were observed to be entrained in this zone until the contact surface came through the area constriction. At $2.480 \mathrm{~ms}$, a Mach disk begins to form at the nozzle exit.

Entrainment of particles behind the ramp, expansion of the flow, and bypass air did not allow for good representation of the particle cloud. In subsequent tests, the square particle wafer was moved back to its original location (see Figure 3.1) and $2.54 \mathrm{~cm}$ high blocks were placed behind the ramps, after the particle wafer. This setup is shown in Figure 4.28.

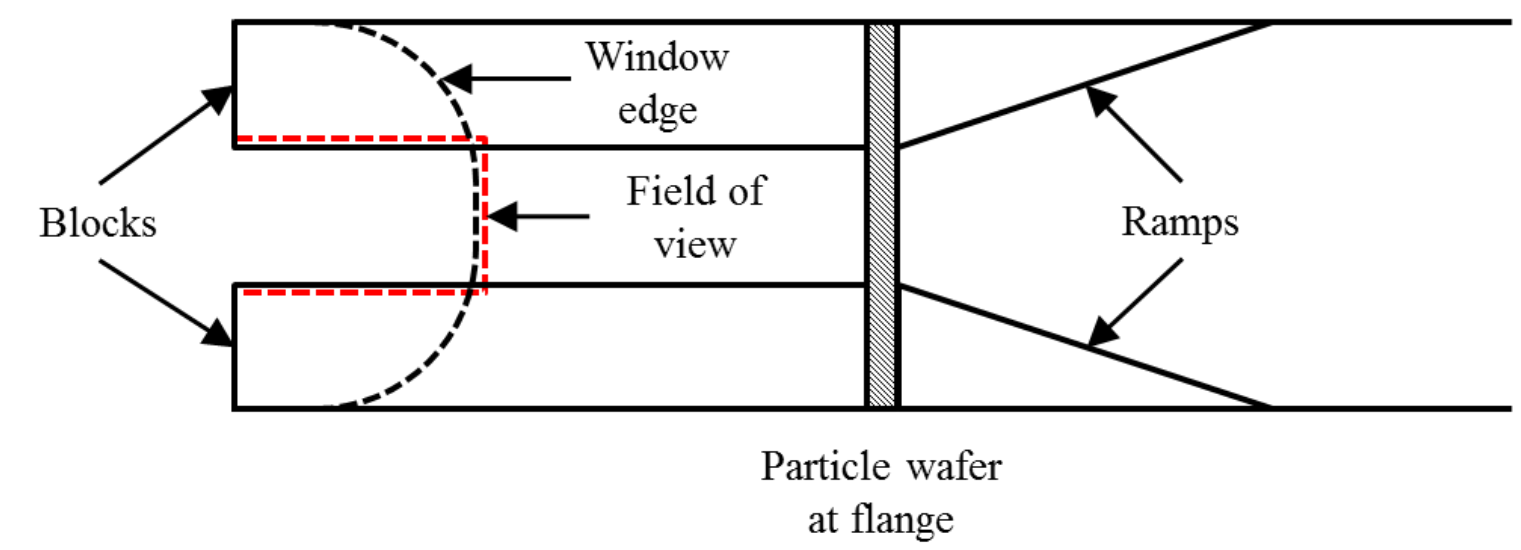

Figure 4.28: Schematic of tube setup for area contraction tests 
The blocks were $61.0 \mathrm{~cm}$ long, effectively reducing the channel cross-section within the optical section to $2.54 \mathrm{~cm}$ by $7.62 \mathrm{~cm}$, or one-third of the original height. The location change of the particle wafer resulted in the ramps being moved out of the field of view. Results from one of these tests are shown in Figure 4.29. 

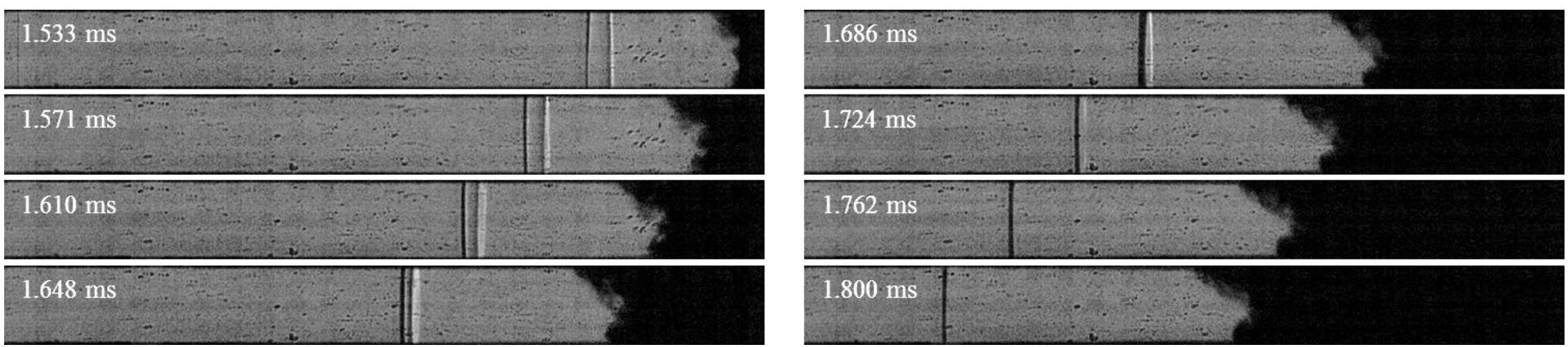

Figure 4.29: Images from 11 bar abs. driver with opposing $18.4^{\circ}$ ramps before particle wafer, channel height of $2.54 \mathrm{~cm}$. Camera frame rate of $105000 \mathrm{fps}$ 
The results seen in Figure 4.29 reveal a similar inverted "V" cloud front that was observed in the full height channel, as wafer boundary conditions were similar. In this configuration the wafer is fixed between the ramp and the block. The incident shock causes shearing through the packed particles of the wafer in the span-wise direction, while edge effects from the die plate come into effect in the normal direction. The cloud front velocity measured from the video was found to average $379 \mathrm{~m} / \mathrm{s}$, much faster than the $320 \mathrm{~m} / \mathrm{s}$ measured in the square cross-section channel. Similar to the low pressure driven section tests, compression waves ahead of the particle cloud coalesce to form a shock wave much earlier than in the standard square channel, but cloud velocities still fall short of the $1000 \mathrm{~m} / \mathrm{s}$ explosive dispersal benchmark. 


\section{Chapter 5}

\section{Summary and Conclusions}

Experiments were performed to observe the dispersion of an initially very dense particle cloud by a shock wave. The goal was to design a clean experiment where no supporting structures were used in the initial geometry of the particle cloud. The approach taken was to compress the particles into a free-standing wafer that could easily be positioned in the shock channel. The high particle volume fraction of the wafer resulted in a solid body response to the shock interaction. No transmitted shock through the particle wafer was observed. Breakup first occurs along the wafer edge in a degradation zone due to shear caused by the fixed wafer boundary condition. In a circular tube, failure of the wafer occurs at local sites, resulting in a series of particle jets that form along the wafer edge. In a square channel the failure first occurs in the corners, where the shear is enhanced, and then progresses to the edges. This failure mechanism produces a nonuniform cloud leading edge. In order to get a one-dimensional cloud dispersion using a particlewafer, a shock wave capable of inducing spallation of the trailing-edge is needed.

Experimental results showed that when impacted by a Mach 2.0 shock wave, a Delrin ${ }^{\circledR}$ block accelerated to an average velocity of $160 \mathrm{~m} / \mathrm{s}$. The reflected shock wave was of similar strength to the theoretical fixed-wall reflection for an incident shock wave of the same Mach number. No shock wave immediately transmitted through the block, but its forward movement produced a series of compression waves that coalesced to form a precursor shock wave.

Using a particle wafer produced a particle cloud upon impact by the incident shock wave. The cloud front velocity was measured to be $321 \mathrm{~m} / \mathrm{s}$. A reflected shock wave was again produced, but it was of a strength slightly lower than the theoretical fixed-wall reflection. A 
series of compression waves once again coalesced downstream to form a precursor shock ahead of the particle cloud. Particle adhered to the inside of the window due to static charge and boundary layer effects, and as a result the rear of the particle cloud was not visible. This also made attempted laser density measurements of the particle cloud impossible. A large pressure pulse was associated with the passage of the particle cloud. The magnitude of the pulse decreased while the pressure rise time increased as the particle wafer ceased to be an impermeable blockage and dispersed. A variation of the initial particle volume fraction found cloud front velocities to be similar for all densities.

The shape of the cloud front was observed to be an inverted "V" shape. Cutting away the edges of the particle wafer eliminated edge effects, but resulted in the incident shock wave diffracting around the wafer, producing a complex three-dimensional flow field. The bypass shock wave tore powder off the wafer edge that was then entrained in a recirculation zone behind it.

Visualization of the upstream face of the particle wafer revealed the wafer initially failed in the corners due to edge effects from the mounting method. Localized wafer failure in the degradation zone allowed small jets, similar to those seen in large-scale multiphase dispersals, to form. Corner failure was the cause of the inverted "V" cloud front.

Experiments were performed in a circular cross-section to eliminate the corner effects. The observed wafer failure was similar to that observed in the square cross-section. Particle jets formed along the circumference of the tube as the wafer failed along the edges. The measured cloud front velocity in the round channel was $294 \mathrm{~m} / \mathrm{s}$. A round Delrin ${ }^{\circledR}$ plug was measured to have the same average velocity as the square block at $160 \mathrm{~m} / \mathrm{s}$. 
A particle wafer was pushed into the round tube, giving the particle wafer a "slip" boundary condition. Wafer failure occurred in a similar mode to the fixed-boundary platemounted wafer, but particle jets also formed away from the tube walls. This produced a uniform cloud front. It was found that the rate of acceleration of the particle wafer was much higher than their Delrin ${ }^{\circledR}$ counterparts due to localized failure of the particle wafer and the convection of the particles in the subsequently formed jet.

Because of the high initial density of the particle wafer, and the relatively weak incident shock wave, spallation of the downstream face did not occur. This made the dispersion a multidimensional phenomenon. Attempts to increase the cloud velocity were successful, but still fell short of a true explosive dispersal.

The main conclusion that can be drawn from this study is that it is very difficult, if not impossible, to generate a truly one-dimensional interaction between a shock wave and a packed granular bed. It is important to remember that failure at the wafer edges obscures the center of the particle wafer. The cloud front edge is made up of only a small fraction of the particles in the wafer and may not give a true indication of the acceleration of the bulk of the particle wafer. Because of this, it would be difficult to use this data to develop an accurate drag correlation to use in the modelling of a dense gas-solid flow. The tests brought to light interesting phenomena related to the failure of an accelerating packed granular bed. In explosives applications, edgeeffects are not present due to the spherical geometry, however the area increase due to the radial movement of the bed would most likely also cause local bed failures and the generation of particle jets. 


\section{Chapter 6}

\section{Recommendations}

While this investigation employed a particle suspension method with no flow obstructing structures, the edge-effects came into play. It is recommended that a particle suspension method be developed that eliminates edge effects, while still allowing no bypass of the incident shock

wave. Also, the problem of particles sticking to the windows must be eliminated to visualize the rear of the particle cloud and to be able to accurately measure the cloud density. High cloud velocities are also desirable to more closely match explosive dispersal events. 


\section{References}

[1] G. Rudiger and A. Chang, "Analysis of non-steady two-phase flow," Pysics of Fluids, vol. 7, no. 11 , pp. $1747-1755,1964$.

[2] J. Thurman, Practical Bomb Scene Investigation, Boca Raton: Taylor \& Francis Group, 2006.

[3] G. Schoffel, M. Bayside, S. Bachmann, C. Mundt and S. Seiler, "Investigation of the acceleration of aluminum particles behind a shock wave using instantaneous Laser Doppler Velocimetry," Shock Waves, vol. 19, no. 2, pp. 125-134, 2009.

[4] V. Tanguay, A. Higgins and F. Zhang, "A Simple Analytic Model for Reactive Particle Ignition in Explosives," Propellant, Explosives, and Pyrotechnics, vol. 32, no. 5, pp. 371384, 2007.

[5] M. Kellenberger, C. Johansen, G. Ciccarelli and F. Zhang, "Dense particle cloud dispersion by a shock wave," in Proceedings of the 28th International Symposium on Shock Waves, Manchester, 2011.

[6] M. Dunning, W. Andrews and K. Jaansaiu, "The Fragmentation of Metal Cylinders Using Thermobaric Explosives," 18 November 2005. [Online]. Available: http://www.dtic.mil/ndia/22ndISB2005/friday/andrews.pdf. [Accessed May 2011].

[7] F. Zhang, D. L. Frost, P. A. Thibault and S. B. Murray, "Explosive dispersal of solid particles," Shock Waves, vol. 10, no. 6, pp. 431-443, 2001.

[8] F. Zhang, "Metalized heterogeneous detonation and dense reactive particle flow," AIP Conference Proceedings, vol. 1426, no. 1, pp. 27-34, 2011.

[9] X. Rogue, G. Rodriguez, J. F. Haas and R. Saurel, "Experimental and numerical investigation of the shock-induced fluidization of a particle bed," Shock Waves, vol. 8, no. 1, pp. 29-45, 1998.

[10] D. L. Frost, Y. Gregoire, S. Goroshin and F. Zhang, "Interfacial instabilities in explosive gas-particle flows," in Proceedings of the 23rd International Colloquium on the Dynamics of Explosive and Reacive Systems, Irvine, 2011.

[11] A. V. Anilkumar, "Experimental Studies of High-Speed Dense Dusty Gases. PhD Thesis," 
California Institute of Technology, Pasedena, 1989.

[12] J. Wagner, S. Baresh, S. Kearney, W. Trott, J. Castaneda, B. Pruett and M. Baer, "Interaction of a planar shock with a dense field of particles," in Proceedings of the 28th International Symposium on Shock Waves, Manchester, 2011.

[13] P. H. Oosthuizen and W. E. Carscallen, An Introduction to Compressible Fluid Flow, 2009.

[14] G. Ciccarelli, MECH 832 Course Notes, 2011.

[15] G. Ben-Dor, O. Igra and T. Elperin, Handbook of Shock Waves, San Diego: Academic Press, 2001.

[16] D. L. Frost, S. Goroshin, J. Levine, R. Ripley and F. Zhang, "Critical Conditions for Ignition of Aluminum Particles in Cylindrical Explosive Charges," AIP Conference Proceedings, vol. 845, no. 1, pp. 972-975, 2006.

[17] D. L. Frost, Y. Gregorie, O. Petel, S. Goroshin and F. Zhang, "Particle jet formation during explosive dispersal of solid particles," 2012.

[18] A. M. Milne, C. Parrish and I. Worland, "Dynamic fragmentaion of blast mitigants," Shock Waves, vol. 20, no. 1, pp. 41-51, 2010.

[19] R. Ripley, "Acceleration and Heating of Metal Particles in Condensed Matter Detonation. PhD Thesis," University of Waterloo, Waterloo, 2010.

[20] F. M. White, Fluid Mechanics, New York: McGraw-Hill, 2008.

[21] R. C. Flagan and J. H. Seinfeld, Fundamentals of Air Pollution Engineering, Englewood Cliffs: Prentice-Hall, 1988.

[22] E. I. Nedderman, "Stokes' Law for Solid Spheres and Spherical Bubbles," Begell House, Inc., 2 February 2011. [Online]. Available: http://www.thermopedia.com/content/1157/. [Accessed 13 September 2012].

[23] E. Loth, Particles, Drops and Bubles: Fluid Dynamics and Numerical Methods, Cambridge University Press, 2010.

[24] M. R. Baer and J. W. Nunziato, "A two-phase mixture theory for the deflagration-todetonation transition (DDT) in reactive granular materials," International Journal of Multiphase Flow, vol. 12, no. 6, pp. 861-889, 1986. 
[25] R. Ripley, F. Zhang and L. Fue-Sang, "Shock Interaction of Metal Particles in Condensed Explosive Detonation," AIP Conference Proceedings, vol. 845, no. 1, pp. 499-502, 2006.

[26] N. Smirnov, "Combustion and detonation in multi-phase media: Initiation of detonation in dispersed-film systemsbehindashockwave," International Journal of Heat and Mass Transfer, vol. 31, no. 4, pp. 779-793, 1988.

[27] C. Johansen, "Experimental and Numerical Investigation of Flame Acceleration in an Obstructed Channel. PhD Thesis," Queen's University, Kingston, 2009.

[28] H. Babinsky and J. K. Harvey, Shock Wave-Boundary-Layer Interactions, New York: Cambridge University Press, 2011. 


\section{Appendix A \\ Uncertainty Analysis}

With all reported numerical values in this thesis, there are associated uncertainties that will be addressed in this section. The key uncertainties discussed will be pressures (fill and shock) and velocities (shock and particle cloud).

Specifications for the pressure gauges used to monitor the shock tube fill pressures stated a measurement accuracy of $0.25 \%$ of the full scale. This gives an uncertainty of \pm 0.017 bar for pressures under 7.89 bar and \pm 0.10 bar for pressures over 7.89 bar.

Uncertainty in reported shock pressures comes from two sources: the piezoelectric transducer and the DAQ device. Transducer specifications state a resolution of $5 \mathrm{mpsi}$. The DAQ device has a resolution of 14 bits, which is equivalent to 1 in $2^{14}$. Coupling this with the $10 \mathrm{~V}$ range of the DAQ card gives a resolution of $0.6 \mathrm{mV}$. The estimated uncertainty from the manual selection of peak pressure on the graphed data is $\pm 2.5 \mathrm{mV}$. Using the nominal transducer sensitivity of $5 \mathrm{mV} / \mathrm{psi}$ and the root-sum-square method, the uncertainty in the shock pressure is \pm 0.05 bar.

The velocity of shock waves was measured by the time of arrival recorded by each pressure transducer and their separation distance. The time of arrival was dependant on the response time of the transducer and the DAQ system the sample rate. From specifications, the pressure transducer response time is $1 \mu$ s and the DAQ card sample rate was $1 \mathrm{MHz}$ for all tests. Uncertainty introduced by the manual selection of the time of arrival from graphed data is estimated to be $\pm 0.25 \mu \mathrm{s}$. This gives an uncertainty in the time of arrival of $\pm 1 \mu \mathrm{s}$. The uncertainty in the downstream distance is taken as the tolerances of the milling machine that created the 
channel, which have been estimated to be $\pm 0.13 \mathrm{~mm}$. This gives an uncertainty in the shock wave velocities of $\pm 1 \mathrm{~m} / \mathrm{s}$.

The calculation of the cloud front velocity was based on data extracted from a video sequence. In the conversion of the grayscale image to a binary black and white image, it is estimated that the cloud front shifted \pm 1 pixel. Since the MATLAB code measures exact pixels to determine the cloud front position, the uncertainty in the cloud front position is dictated by the binary conversion uncertainty and an estimated uncertainty of \pm 2 pixels for the edge offset (pixels between the upstream window edge and the camera field of view upstream edge). The edge offset was measured manually as well as the pixel density, resulting in \pm 26 pixels $/ \mathrm{m}$ for the pixel density uncertainty. The uncertainty of the window edge location was taken as the tolerances of the milling machine that created the channel, which have been estimated to be $\pm 0.13 \mathrm{~mm}$. This gives an uncertainty in the cloud front velocity of $\pm 1 \mathrm{~m} / \mathrm{s}$. While this uncertainty is low, the uncertainty derived from the standard deviation of average cloud front velocities was found to be $\pm 12 \mathrm{~m} / \mathrm{s}$. This can be attributed to the stochastic nature of the particle cloud that forms. 


\section{Appendix B}

\section{MATLAB Cloud Tracking Code}

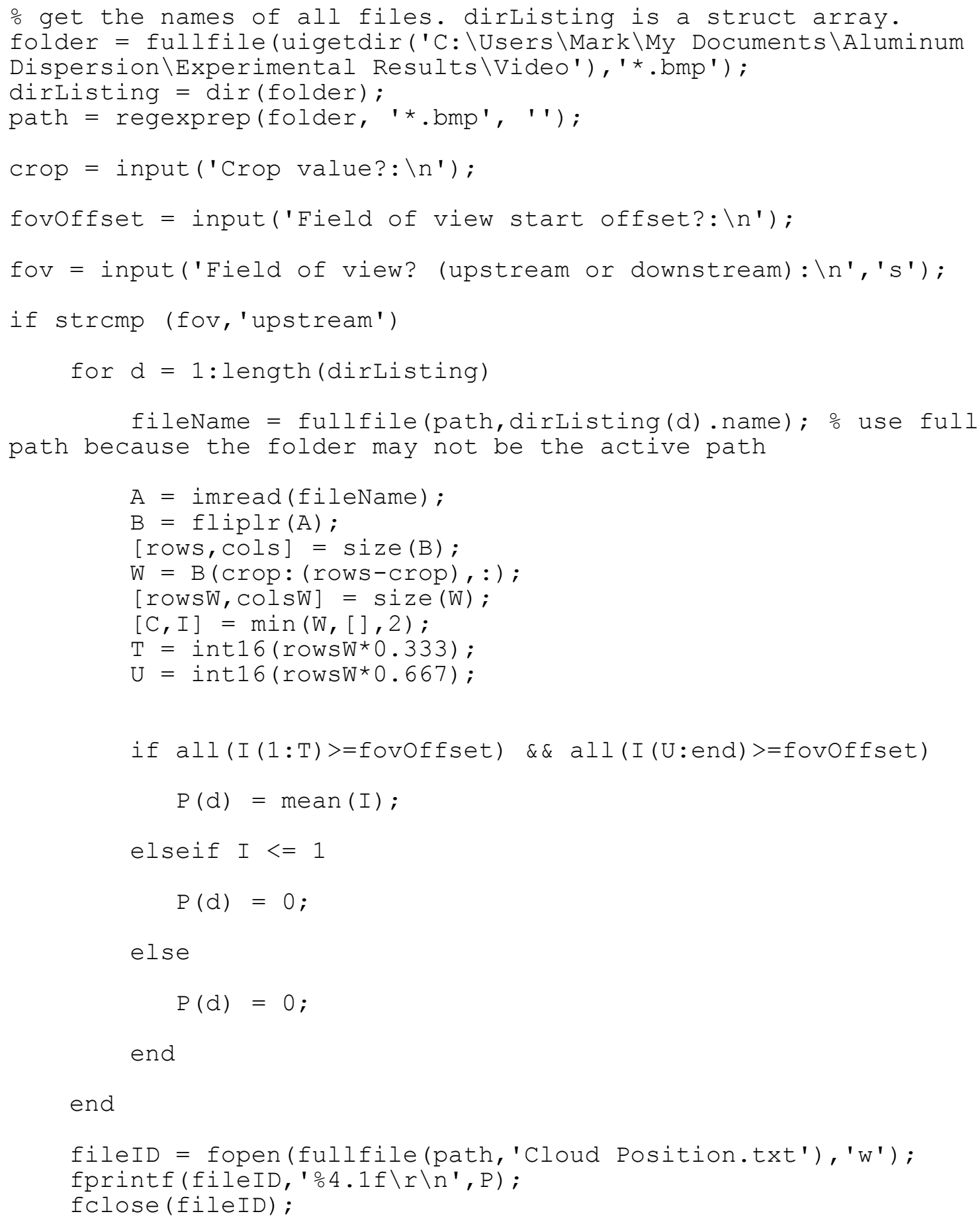




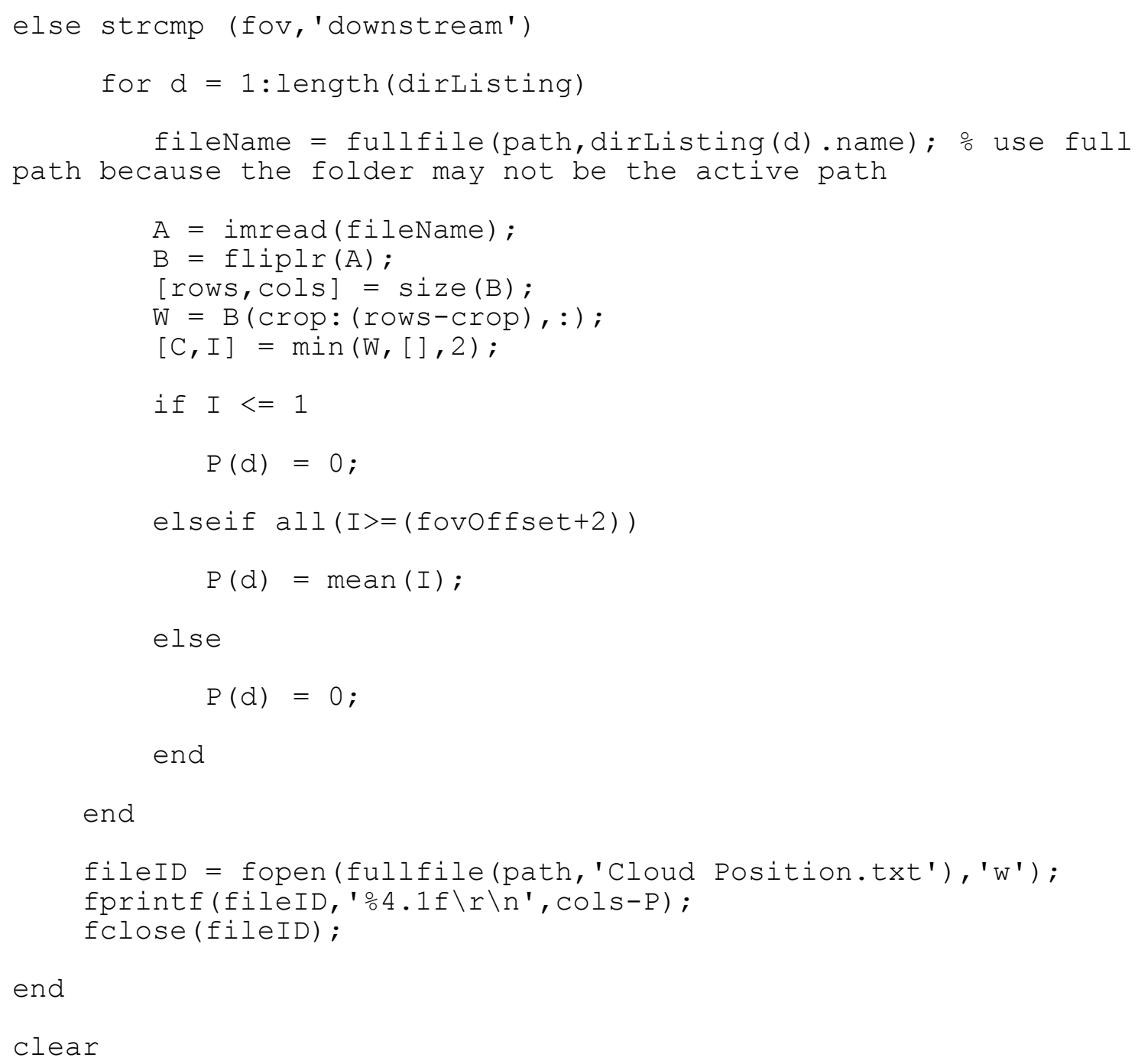

